\title{
Search for long-lived particles using delayed photons in proton-proton collisions at $\sqrt{s}=13 \mathrm{TeV}$
}

\author{
A. M. Sirunyan et al. \\ (CMS Collaboration)
}

(Received 13 September 2019; published 9 December 2019)

\begin{abstract}
A search for long-lived particles decaying to photons and weakly interacting particles, using protonproton collision data at $\sqrt{s}=13 \mathrm{TeV}$ collected by the CMS experiment in 2016-2017 is presented. The data set corresponds to an integrated luminosity of $77.4 \mathrm{fb}^{-1}$. Results are interpreted in the context of supersymmetry with gauge-mediated supersymmetry breaking, where the neutralino is long-lived and decays to a photon and a gravitino. Limits are presented as a function of the neutralino proper decay length and mass. For neutralino proper decay lengths of $0.1,1,10$, and $100 \mathrm{~m}$, masses up to 320, 525, 360, and $215 \mathrm{GeV}$ are excluded at $95 \%$ confidence level, respectively. We extend the previous best limits in the neutralino proper decay length by up to one order of magnitude, and in the neutralino mass by up to $100 \mathrm{GeV}$.
\end{abstract}

DOI: $10.1103 /$ PhysRevD.100.112003

\section{INTRODUCTION}

The results of a search for long-lived particles (LLP) decaying to a photon and a weakly interacting particle are presented. Neutral particles with long lifetimes are predicted in many models of physics beyond the standard model (SM). In this paper, a benchmark scenario of supersymmetry (SUSY) [1-14] with gauge-mediated SUSY breaking (GMSB) [15-23] is employed, commonly referred to as the "Snowmass Points and Slopes 8" (SPS8) benchmark model [24]. In this scenario, pair-produced squarks and gluinos undergo cascade decays as shown in Fig. 1, and eventually produce the lightest SUSY particle (LSP), the gravitino $(\tilde{G})$, which is stable and weakly interacting. The phenomenology of such decay chains is primarily determined by the nature of the next-to-lightest SUSY particle (NLSP). In the SPS8 benchmark, the NLSP is the lightest neutralino, $\tilde{\chi}_{1}^{0}$, and the mass of the NLSP is linearly related to the effective scale of SUSY breaking, $\Lambda[15,25]$.

In the SPS 8 model, $\Lambda$ is a free parameter whose value determines the primary production mode and decay rate of SUSY particles. Depending on the value of $\Lambda$, the coupling of the NLSP to the gravitino could be very weak and lead to long NLSP lifetimes. The dominant decay mode of the NLSP is to a photon and a gravitino, resulting in a final

*Full author list given at the end of the article.

Published by the American Physical Society under the terms of the Creative Commons Attribution 4.0 International license. Further distribution of this work must maintain attribution to the author(s) and the published article's title, journal citation, and DOI. Funded by SCOAP ${ }^{3}$. state with one or two photons and missing transverse momentum $\left(p_{\mathrm{T}}^{\text {miss }}\right)$. The dominant squark-pair and gluinopair production modes also result in additional energetic jets. If the NLSP has a proper decay length that is a significant fraction of the radius of the CMS tracking volume (about $1.2 \mathrm{~m}$ ), then the photons produced at the secondary vertex tend to exhibit distinctive features. Because of their production at displaced vertices and their resulting trajectories, the photons have significantly delayed arrival times (order of ns) at the CMS electromagnetic calorimeter (ECAL) compared to particles produced at the primary vertex and traveling at the speed of light. They also enter the ECAL at non-normal impact angles.

The present search makes use of these features to identify potential signals of physics beyond the SM. We select events with one or two displaced or delayed photons, and three or more jets. Signal events are expected to produce large $p_{\mathrm{T}}^{\text {miss }}$ as the LSP escapes the detector volume without detection. In the case of very long-lived NLSPs, one of the NLSPs may completely escape the detector, further increasing the $p_{\mathrm{T}}^{\text {miss }}$. Previously, similar searches for LLPs decaying to displaced or delayed photons have been performed by the CMS [26] and ATLAS [27] Collaborations using LHC collisions at a center-of-mass energies of 7 and $8 \mathrm{TeV}$, respectively. Past LHC searches for invisible Higgs boson decays in association with photons [28] also have sensitivity to such models.

\section{THE CMS DETECTOR}

The central feature of the CMS apparatus is a superconducting solenoid of $6 \mathrm{~m}$ internal diameter, providing a magnetic field of $3.8 \mathrm{~T}$. Within the solenoid volume are a silicon pixel and strip tracker, a lead tungstate crystal 

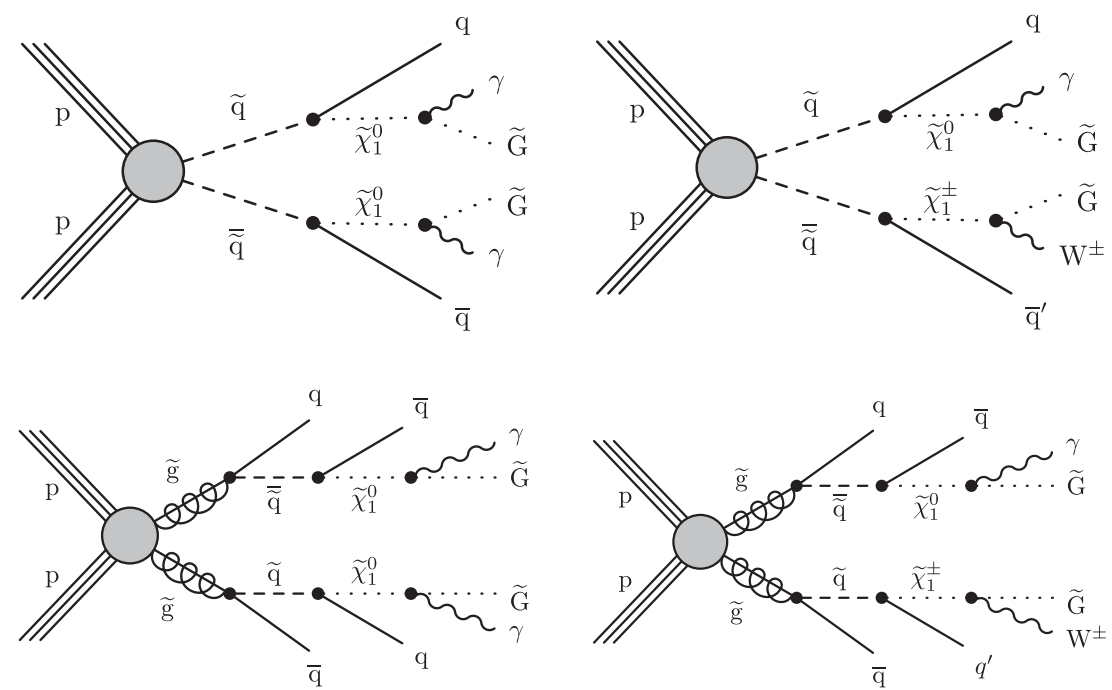
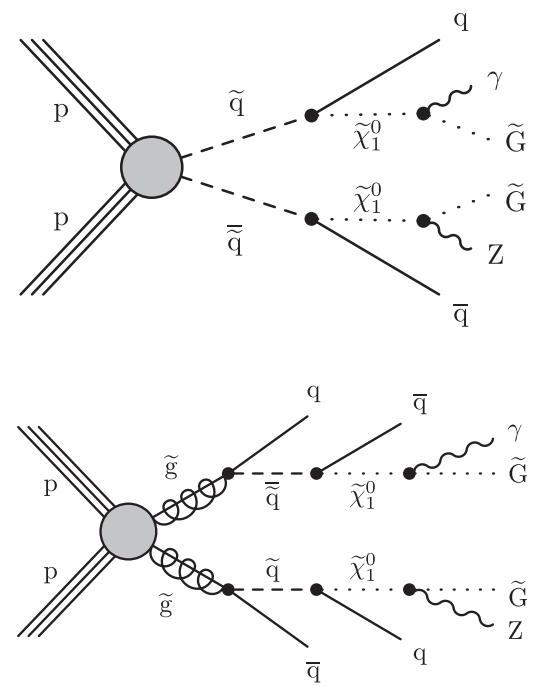

FIG. 1. Example Feynman diagrams for SUSY processes that result in diphoton (left) and single photon (middle and right) final states via squark (upper) and gluino (lower) pair-production at the LHC.

ECAL, and a brass and scintillator hadron calorimeter (HCAL), each composed of a barrel and two endcap sections. Forward calorimeters extend the pseudorapidity coverage provided by the barrel and endcap detectors. Muons are measured in gas-ionization detectors embedded in the steel flux-return yoke outside the solenoid.

The ECAL is highly granular and consists of 61200 crystals in the barrel region, each with an area of approximately $2.2 \times 2.2 \mathrm{~cm}^{2}$ corresponding to roughly $0.0174 \times$ 0.0174 in $\eta-\phi$ space, where $\eta$ is the pseudorapidity and $\phi$ the azimuthal angle (in radians) of the coordinate system [29]. Each of the two endcap sections consist of 7324 crystals, each crystal having an area of $2.68 \times 2.68 \mathrm{~cm}^{2}$. A typical electromagnetic shower spans approximately 10 crystals with energy deposits above noise threshold. The barrel and endcap ECAL components cover the regions with $|\eta|<1.5$ and $1.5<|\eta|<2.5$, respectively. The best possible time resolution for each ECAL channel is measured to be between 70 and $100 \mathrm{ps,} \mathrm{depending} \mathrm{on} \mathrm{detector} \mathrm{aging.}$

The first level of the CMS trigger system [30], composed of custom hardware processors, uses information from the calorimeters and muon detectors to select the most interesting events in a fixed time interval of less than $4 \mu \mathrm{s}$. The high-level trigger (HLT) processor farm further decreases the event rate from around $100 \mathrm{kHz}$ to less than $1 \mathrm{kHz}$, before data storage. A more detailed description of the CMS detector, together with a definition of the coordinate system used and the relevant kinematic variables, can be found in Ref. [29].

\section{EVENT SAMPLES}

This analysis uses data sets of proton-proton $(p p)$ collisions collected by the CMS experiment at the LHC in 2016 and 2017, corresponding to integrated luminosities of 35.9 and $41.5 \mathrm{fb}^{-1}$, respectively. Simulated samples are used to study the SM background and signal contributions, primarily for the purpose of optimizing the event selection and the binning in the photon time and $p_{\mathrm{T}}^{\text {miss }}$ observables. The MADGRAPH5_aMC@NLO v2.2.2 generator [31] is used at next-to-leading order (NLO) in quantum chromodynamics (QCD) to simulate events originating from single top quark and top quark pair production, and at leading order (LO) to simulate events originating from QCD multijet, $\gamma+$ jets, $\mathrm{W}+$ jets, and $\mathrm{Z}+$ jets production. Simulated samples of diphoton events are generated using SHERPA v2.2.4 [32,33], and include Born processes with up to three additional jets, as well as box processes at $\mathrm{LO}$ precision. The particle spectra of each GMSB SPS8 signal model are tabulated in a SUSY Les Houches accord (SLHA) file using ISASUGRA as part of ISAJET v7.87 [34]. The SLHA files are then used to generate benchmark signal model samples using PYTHIA v8.212 (v8.230) [35] for the 2016 (2017) data analysis.

For all simulated samples discussed above, the fragmentation and parton showering are modeled using PYTHIA v8.212 with the CUETP8M1 underlying event tune [36,37] (PYTHIA v8.230 with the CP5 [38] tune) for the 2016 (2017) data analysis. The NNPDF3.0 [39] and NNPDF3.1 [40] parton distribution function (PDF) sets are used for the 2016 and 2017 simulated samples, respectively. The signal and background samples are processed through a simulation of the CMS detector based on GEANT4 [41] and are reconstructed with the same algorithms as used for data. Additional $p p$ interactions in the same or adjacent bunch crossings, referred to as pileup, are also simulated.

\section{TRIGGER AND EVENT SELECTION}

The unique signature of delayed photons is best exploited with specialized triggers and dedicated photon 
reconstruction and identification criteria. There is a difference between the search selections for the 2016 and 2017 data sets, primarily because of the introduction of a targeted HLT algorithm implemented for the 2017 data set, which superseded a general diphoton trigger used for the 2016 data set.

\section{A. Trigger selection}

For the 2016 data set, events are selected by the standard diphoton trigger, requiring transverse momenta $\left(p_{\mathrm{T}}\right)$ larger than 42 and $25 \mathrm{GeV}$ for the leading and subleading photons, respectively. Loose identification criteria are imposed on the photon shower width in the ECAL and on the ratio of the energies recorded in the ECAL and HCAL to reduce the rate of background from jets misidentified as photons.

For the 2017 data set, a dedicated HLT algorithm was developed to select events with a single photon satisfying requirements consistent with production at a displaced vertex. Such photons tend to strike the front face of the barrel ECAL at a non-normal incidence angle, resulting in a more elliptical electromagnetic shower in the $\eta-\phi$ plane [26]. In addition to standard requirements on the shower width and electromagnetic to hadronic energy ratio, requirements on the major and minor axes of the shower are also imposed. This allows the identification of the elliptical shower shape, described in greater detail in Sec. IV B. Loose requirements on the amount of energy around the direction of the photon in the CMS subdetectors (isolation) are also imposed on trigger photon candidates, and the photon $p_{\mathrm{T}}$ is required to exceed $60 \mathrm{GeV}$. Electrons misidentified as photons are suppressed by requiring the candidate photon to be geometrically isolated from charged-particle tracks. Relaxing the trigger requirement from two photons to only one photon increases the background rate, and in order to reduce the trigger rate to a level acceptable for the operation of the HLT the scalar $p_{\mathrm{T}}$ sum of all jets $\left(H_{\mathrm{T}}\right)$ is required to exceed $350 \mathrm{GeV}$. For signals with neutralino proper decay length larger than $10 \mathrm{~m}$, the signal acceptance is improved by about a factor of two compared to the 2016 data set.

\section{B. Object reconstruction and selection}

A particle-flow (PF) algorithm [42] is used to reconstruct and identify each individual particle in an event using an optimized combination of information from the various elements of the CMS detector. The candidate vertex with the largest value of summed physics-object $p_{\mathrm{T}}^{2}$ is taken to be the primary $p p$ interaction vertex. The physics objects are the jets, clustered using the jet finding algorithm $[43,44]$ with the tracks assigned to candidate vertices as inputs, and the associated missing transverse momentum, taken as the negative vector sum of the $p_{\mathrm{T}}$ of those jets.

Photon candidates are reconstructed from energy clusters in the ECAL [45] and identified based on the transverse shower width, the hadronic to electromagnetic energy ratio, and the degree of isolation from charged particle tracks. Photons are required to satisfy $|\eta|<2.5$ and to not fall in the transition region between the barrel and endcap of the ECAL $(1.444<|\eta|<1.566)$, where the photon reconstruction is not optimal. For the 2016 data set, photon candidates that share the same energy cluster as an identified electron associated with the primary vertex are vetoed following the procedure detailed in Ref. [45]. To remain consistent with the HLT selection, photons matched geometrically to charged-particle tracks are vetoed for the 2017 data set as well.

Because of algorithms designed to reject noise and out-of-time pileup, the default photon reconstruction vetoes photons delayed by more than $3 \mathrm{~ns}$. To evade this veto, a second set of out-of-time (OOT) photons is therefore defined, in which the clustering starts from ECAL deposits whose signals are delayed by more than 3 ns. The remainder of the reconstruction algorithm for OOT photons is identical to the standard photon reconstruction described in the previous paragraph. In addition to being delayed, signal photons tend to impact the front face of the barrel ECAL at a non-normal incidence angle, and yield electromagnetic showers that are more elliptical in the $\eta-\phi$ plane. To make use of this discriminating feature, we define the OOT photon identification criteria including selection requirements on the $S_{\text {major }}$ and $S_{\text {minor }}$ observables defined as:

$$
\begin{aligned}
S_{\text {major }}= & \frac{S_{\phi \phi}+S_{\eta \eta}+\sqrt{\left(S_{\phi \phi}-S_{\eta \eta}\right)^{2}+4 S_{\eta \phi}^{2}}}{2} \\
S_{\text {minor }}= & \frac{S_{\phi \phi}+S_{\eta \eta}-\sqrt{\left(S_{\phi \phi}-S_{\eta \eta}\right)^{2}+4 S_{\eta \phi}^{2}}}{2}
\end{aligned}
$$

where $S_{\phi \phi}, S_{\eta \eta}$, and $S_{\eta \phi}$ are the second central moments of the spatial distribution of the energy deposits in the ECAL in $\eta-\phi$ coordinates, and are proportional to the squared lengths of the semimajor and semiminor axes of the elliptical shower shape. The full set of criteria for the OOT photon selection additionally includes requirements on the transverse shower width and isolation and was obtained through a separate optimization that maximizes the discrimination between displaced signal photons and background photons associated with the primary vertex.

Hadronic jets are reconstructed by clustering PF candidates using the anti- $k_{\mathrm{T}}$ algorithm with a distance parameter of $0.4[43,44]$. Further details of the performance of the jet reconstruction can be found in Ref. [46]. Jets used in any selection of this analysis are required to have $p_{\mathrm{T}}>30 \mathrm{GeV}$ and $|\eta|<3.0$.

The negative vector $p_{\mathrm{T}}$ sum of all the PF candidates in an event is defined as $\vec{p}_{\mathrm{T}}^{\text {miss }}$, and its magnitude is denoted as $p_{\mathrm{T}}^{\text {miss }}$ [47]. The $\vec{p}_{\mathrm{T}}^{\text {miss }}$ is modified to account for corrections to the energy scale of the reconstructed jets in the event. 
Because OOT photons are not part of the standard PF candidate reconstruction used to compute the $\vec{p}_{\mathrm{T}}^{\text {miss }}$, we correct the $\vec{p}_{\mathrm{T}}^{\text {miss }}$ by adding the negative momentum of an OOT photon if it is selected in the event. Anomalous high$p_{\mathrm{T}}^{\text {miss }}$ events can arise because of a variety of reconstruction failures, detector malfunctions, or noncollision backgrounds. Filters for vetoing such anomalous events are applied [47].

\section{Photon time reconstruction}

Photons from signal events tend to arrive at the ECAL up to $10 \mathrm{~ns}$ later than particles produced at the primary vertex. Therefore measuring the photon time of arrival delay with respect to a photon produced at the primary vertex and traveling at the speed of light helps to discriminate between signal and background. The time of arrival of a photon at the ECAL, $t_{\mathrm{ECAL}}$, is calculated based on a weighted sum of the arrival times reconstructed from the signal pulse in each ECAL crystal comprising the photon cluster:

$$
t_{\mathrm{ECAL}}=\frac{\sum_{i} \frac{t_{\mathrm{ECAL}}^{i}}{\sigma_{i}^{2}}}{\sum_{i} \frac{1}{\sigma_{i}^{2}}},
$$

where $t_{\mathrm{ECAL}}^{i}$ is the timestamp of the signal pulse in crystal $i$ [48]. The estimated time resolution of the signal pulse in crystal $i$ is $\sigma_{i}$ and is parametrized as:

$$
\sigma_{i}^{2}=\left(\frac{N}{A_{i} / \sigma_{\mathrm{N}_{i}}}\right)^{2}+C^{2},
$$

where $A_{i}$ is the amplitude of the signal detected by crystal $i$, $\sigma_{\mathrm{N}_{i}}$ is the pedestal noise for crystal $i$, and $N$ and $C$ are constants fitted from a dedicated measurement of the time resolution of the crystal sensors.

To measure the crystal sensor time resolution, we follow a procedure similar to that described in Refs. $[48,49]$. We first apply a very loose selection on photons using $S_{\text {major }}$ and $S_{\text {minor }}$ in order to reject jets. Pairs of crystals from the same photon cluster are selected by requiring that their energies are within $20 \%$ of each other, are nearest neighbors either in the $\eta$ or $\phi$ directions, and are within the same $5 \times 5$ grid of crystals defining a trigger tower. The distributions of time differences measured in such crystal pairs are fitted using Gaussian functions in bins of the effective amplitude $A_{\text {eff }} / \sigma_{\mathrm{N}}$, and the standard deviation of each fitted Gaussian function is trended as a function of $A_{\text {eff }} / \sigma_{\mathrm{N}}$. The effective amplitude is obtained combining the signals in the two crystals and is denoted by:

$$
A_{\mathrm{eff}} / \sigma_{\mathrm{N}}=\frac{\left(A_{1} / \sigma_{\mathrm{N}_{1}}\right)\left(A_{2} / \sigma_{\mathrm{N}_{2}}\right)}{\sqrt{\left(A_{1} / \sigma_{\mathrm{N}_{1}}\right)^{2}+\left(A_{2} / \sigma_{\mathrm{N}_{2}}\right)^{2}}} .
$$

The results for the 2016 and 2017 data sets are shown in Fig. 2. These resolution measurements are fitted with the

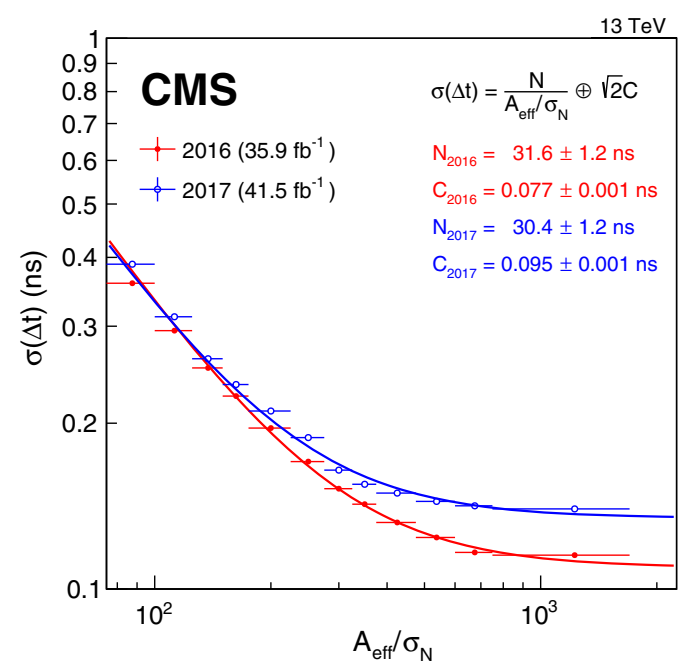

FIG. 2. The time resolution between two neighboring ECAL crystals as a function of the effective amplitudes of the signals in the two crystals for the 2016 and 2017 data sets. The lines shown reflect the fits described in the text. The horizontal bars on the data represent the bin widths, which are treated as uncertainties in the fit.

functional form given by Eq. (3), and the $N$ and $C$ parameters are extracted and summarized in Table I. These parameters are then used to calculate the weights for the photon timestamp in Eq. (2). The observed worsening of the constant term to the time resolution in 2017 may be due to a progressive loss of transparency of the crystals from radiation damage.

To calibrate the photon timestamp response, electrons from $Z \rightarrow e^{+} e^{-}$decays with an invariant mass between 60 and $150 \mathrm{GeV}$ are reconstructed as photons. For each such photon candidate, the $t_{\mathrm{ECAL}}$ is adjusted for the time-offlight between the primary vertex and the location of the impact of the photon on the front face of ECAL. The timestamp for each photon is recorded, and the mean and RMS parameters of the resulting distribution are extracted as a function of the photon energy. The time response mean is adjusted to zero for both data and simulation, and the timestamps in the simulated events are smeared by an additional Gaussian-distributed random variable such that the resolution in simulation matches that measured in data. The calibrated photon arrival time is denoted as $t_{\gamma}$. These calibrations are applied to simulated signal samples in order to accurately predict the signal response, and their uncertainties are propagated to the predicted shape of the $t_{\gamma}$

TABLE I. The fitted ECAL timing resolution parameters for the 2016 and 2017 data sets.

\begin{tabular}{lcc}
\hline \hline Parameters & 2016 Data set & 2017 Data set \\
\hline $\mathrm{N}$ & $31.6 \pm 1.2 \mathrm{~ns}$ & $30.4 \pm 1.2 \mathrm{~ns}$ \\
$\mathrm{C}$ & $0.077 \pm 0.001 \mathrm{~ns}$ & $0.095 \pm 0.001 \mathrm{~ns}$ \\
\hline \hline
\end{tabular}



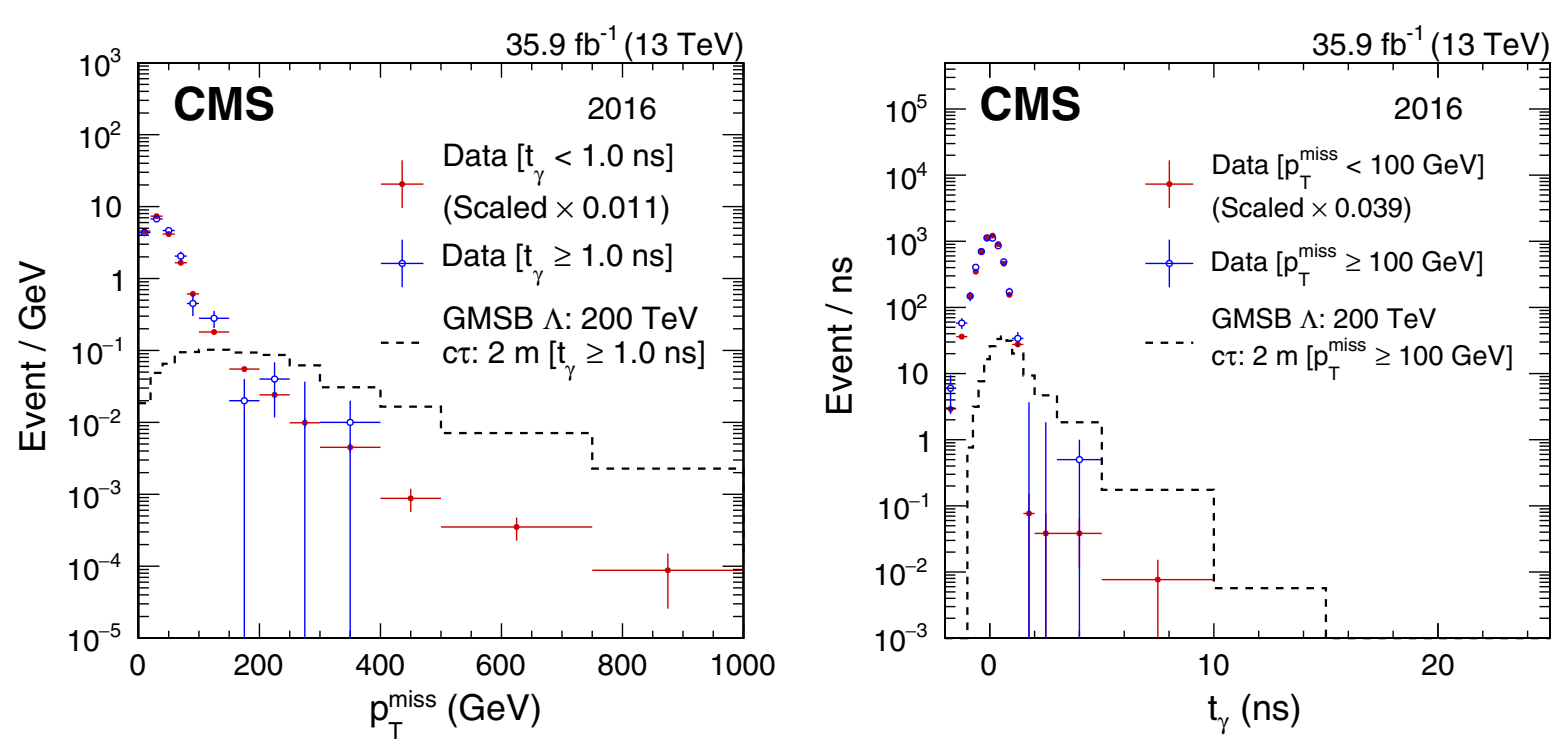

FIG. 3. The $p_{\mathrm{T}}^{\text {miss }}$ (left) and $t_{\gamma}$ (right) distributions for the 2016 event selection, shown for data and a representative signal benchmark (GMSB: $\Lambda=200 \mathrm{TeV}, c \tau=2 \mathrm{~m}$ ). The $p_{\mathrm{T}}^{\text {miss }}$ distribution for data is separated into events with $t_{\gamma} \geq 1 \mathrm{~ns}$ (blue, darker) and $t_{\gamma}<1 \mathrm{~ns}$ (red, lighter), scaled to match the total number of events with $t_{\gamma} \geq 1 \mathrm{~ns}$. The $t_{\gamma}$ distribution for data is separated into events with $p_{\mathrm{T}}^{\text {miss }} \geq 100 \mathrm{GeV}$ (blue, darker) and $p_{\mathrm{T}}^{\text {miss }}<100 \mathrm{GeV}$ (red, lighter), scaled to match the total number of events with $p_{\mathrm{T}}^{\text {miss }} \geq 100 \mathrm{GeV}$. The signal (black, dotted) is shown in the left plot only for events with $t_{\gamma} \geq 1 \mathrm{~ns}$, and in the right plot only for events with $p_{\mathrm{T}}^{\text {miss }} \geq 100 \mathrm{GeV}$. The entries in each bin are normalized by the bin width. The horizontal bars on data indicate the bin boundaries. The last bin in each plot includes overflow events.

distribution for the signal as a systematic uncertainty. The time resolution of a single photon candidate is roughly $400 \mathrm{ps}$. The resolution is constant up to a photon timestamp of $25 \mathrm{~ns}$, the upper boundary of $t_{\gamma}$ used during the signal extraction.

\section{Event selection}

Events with at least one photon in the barrel region of the detector $(|\eta|<1.444)$ with $p_{\mathrm{T}}$ larger than $70 \mathrm{GeV}$ are selected. Standard photons [45] and OOT photons are required to pass the "tight" working points. Both photon identifications are tuned to have an average efficiency of about $70 \%$. Furthermore, a displaced photon identification requirement based on the $S_{\text {major }}$ and $S_{\text {minor }}$ variables is imposed. The calibrated arrival time of this tight photon, $t_{\gamma}$, is used as one of the final discriminating observables to distinguish signal from background. For the dominant squark-pair and gluino-pair production modes shown in Fig. 1, the NLSP is generally produced in association with several jets, and therefore we also require events to have three or more jets with $p_{\mathrm{T}}$ larger than $30 \mathrm{GeV}$.

In order to remain compatible with the respective HLT selection, slightly different event selection criteria are imposed on the 2016 and 2017 data sets. For the 2016 data set, triggered by a diphoton HLT, a second photon with $p_{\mathrm{T}}$ larger than $40 \mathrm{GeV}$ is required to match the analogous HLT requirement. For the 2017 data set, the first category, referred to as the $2017 \gamma$ category, requires events with no subleading photon or events where the subleading photon does not pass the photon identification criteria. The second category requires events to have a subleading photon satisfying the photon identification criteria, and is referred to as the $2017 \gamma \gamma$ category. The second-photon requirement helps to reduce background by one to two orders of magnitude, while the signal yield remains high for low to intermediate lifetimes. Finally, for the 2017 data set, the $H_{\mathrm{T}}$ is required to be larger than $400 \mathrm{GeV}$ in order to match the requirements of the HLT and to reach the plateau of the trigger efficiency.

For the 2016 and $2017 \gamma \gamma$ analyses, for a given neutralino proper decay length, the signal yield increases as a function of the SUSY breaking scale, $\Lambda$, by roughly a factor of two over the range considered for this analysis ( $\Lambda$ from 100 to $400 \mathrm{TeV}$ ). The product of signal efficiency and acceptance for the lowest $\Lambda$ is roughly $10.0 \pm 0.1 \%$ and $0.15 \pm 0.01 \%$ for neutralino proper decay lengths of 0.1 and $100 \mathrm{~m}$, respectively. For the $2017 \gamma$ analysis, the product of signal efficiency and acceptance varies as a function of $\Lambda$ from $5.5 \pm 0.1$ to $10.4 \pm 0.2 \%$ for a neutralino proper decay length of $0.1 \mathrm{~m}$, and from $0.22 \pm 0.03$ to $0.65 \pm 0.05 \%$ for a neutralino proper decay length of $100 \mathrm{~m}$. These trends can be explained by the harder photon spectrum and increase in jet activity that result from an increase in $\Lambda$, while an increase in the neutralino proper decay length results in either one or both of the NLSPs decaying outside the fiducial region of ECAL.

Figures 3 and 4 show the $p_{\mathrm{T}}^{\text {miss }}\left(t_{\gamma}\right)$ distribution in data for low and high $t_{\gamma}$ (low and high $p_{\mathrm{T}}^{\text {miss }}$ ), for the 2016, 2017 , 

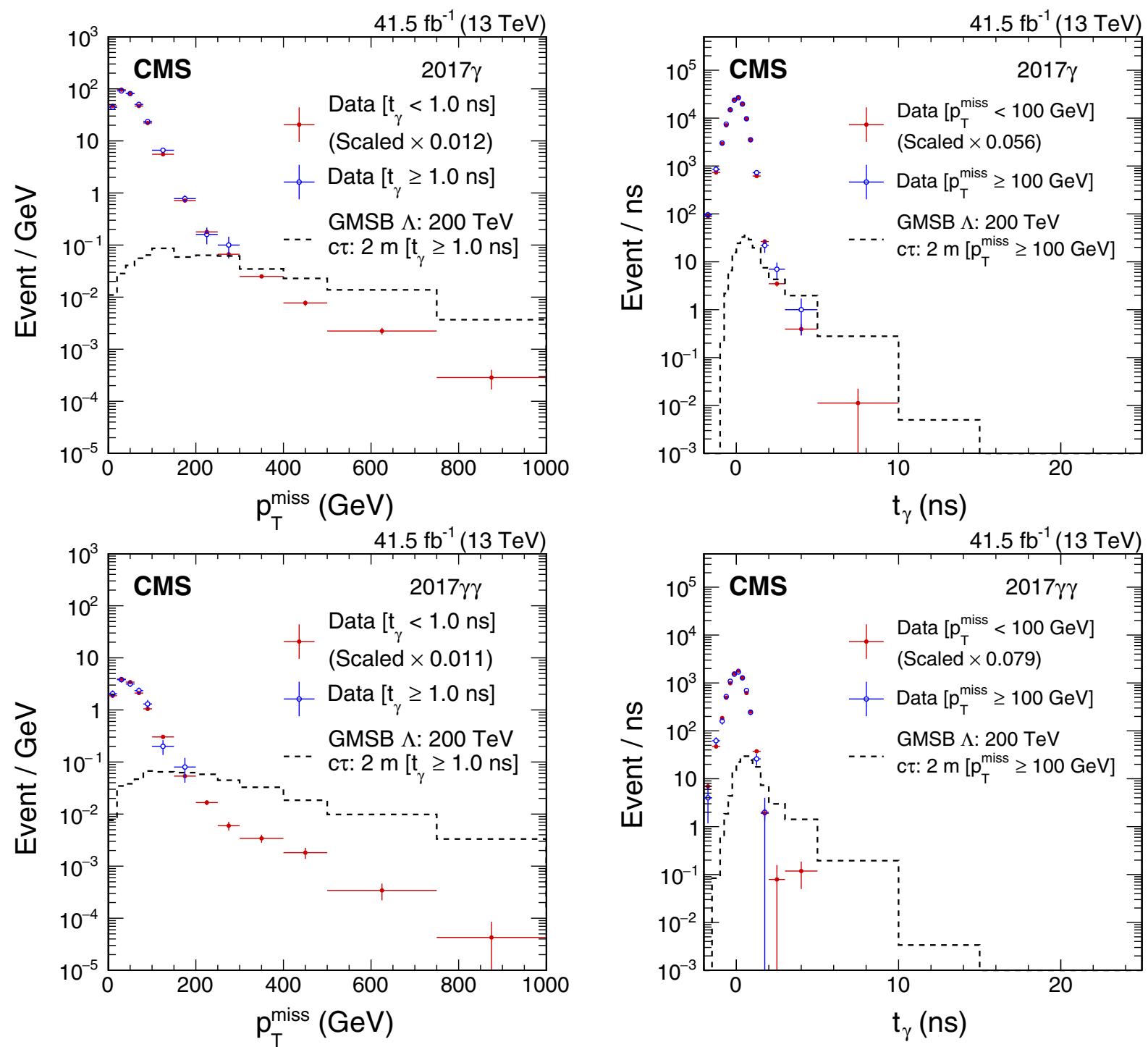

FIG. 4. The $p_{\mathrm{T}}^{\text {miss }}$ (left) and $t_{\gamma}$ (right) distributions for the $2017 \gamma$ (upper row) and $2017 \gamma \gamma$ (lower row) event selections shown for data and a representative signal benchmark (GMSB: $\Lambda=200 \mathrm{TeV}, c \tau=2 \mathrm{~m}$ ). The $p_{\mathrm{T}}^{\text {miss }}$ distribution for data is separated into events with $t_{\gamma} \geq 1 \mathrm{~ns}$ (blue, darker) and $t_{\gamma}<1 \mathrm{~ns}$ (red, lighter), scaled to match the total number of events with $t_{\gamma} \geq 1 \mathrm{~ns}$. The $t_{\gamma}$ distribution for data is separated into events with $p_{\mathrm{T}}^{\text {miss }} \geq 100 \mathrm{GeV}$ (blue, darker) and $p_{\mathrm{T}}^{\text {miss }}<100 \mathrm{GeV}$ (red, lighter), scaled to match the total number of events with $p_{\mathrm{T}}^{\text {miss }} \geq 100 \mathrm{GeV}$. The signal (black, dotted) is shown in the left plots only for events with $t_{\gamma} \geq 1 \mathrm{~ns}$, and in the right plots only for events with $p_{\mathrm{T}}^{\text {miss }} \geq 100 \mathrm{GeV}$. The entries in each bin are normalized by the bin width. The horizontal bars on data indicate the bin boundaries. The last bin in each plot includes overflow events.

and $2017 \gamma \gamma$ event selections. In addition, the distribution of events for a representative signal point (GMSB: $\Lambda=200 \mathrm{TeV}, c \tau=2 \mathrm{~m}$ ) is also shown, scaled by the product of the production cross section and the integrated luminosity in the regions most sensitive to this signal benchmark: large $p_{\mathrm{T}}^{\text {miss }}$ and $t_{\gamma}$.

\section{SIGNAL EXTRACTION AND BACKGROUND ESTIMATION}

The $p_{\mathrm{T}}^{\text {miss }}$ and $t_{\gamma}$ variables are used as the final discriminating observables to distinguish signal from background.
Standard model background events can populate the signalenriched regions with large values of $p_{\mathrm{T}}^{\text {miss }}$ and $t_{\gamma}$ because of imperfect resolution. Four bins are defined based on the values of the $p_{\mathrm{T}}^{\text {miss }}$ and $t_{\gamma}$ observables. Bin $\mathrm{A}$ has low $p_{\mathrm{T}}^{\text {miss }}$ and low $t_{\gamma}$; bin $\mathrm{B}$ has high $p_{\mathrm{T}}^{\text {miss }}$ and low $t_{\gamma}$; bin $\mathrm{C}$ has high $p_{\mathrm{T}}^{\text {miss }}$ and high $t_{\gamma}$; and bin $\mathrm{D}$ has low $p_{\mathrm{T}}^{\text {miss }}$ and high $t_{\gamma}$. Signals with large lifetimes are concentrated in bin C, while signals with shorter lifetimes tend to occupy bin B. In contrast, backgrounds are concentrated in bin A. In general, bin $\mathrm{C}$ is the most sensitive, with largest signal to background ratio. After the offline selection is applied, the main 
TABLE II. The optimized bin boundaries for $t_{\gamma}$ (first number, in units of ns) and $p_{\mathrm{T}}^{\text {miss }}$ (second number, in units of GeV), for different GMSB SPS8 signal model benchmark points considered in the search and for each data set category.

\begin{tabular}{|c|c|c|c|c|c|c|}
\hline \multirow[b]{2}{*}{$c \tau(\mathrm{m})$} & \multicolumn{3}{|c|}{$\Lambda \leq 300 \mathrm{TeV}$} & \multicolumn{3}{|c|}{$\Lambda>300 \mathrm{TeV}$} \\
\hline & 2016 & $2017 \gamma$ & $2017 \gamma \gamma$ & 2016 & $2017 \gamma$ & $2017 \gamma \gamma$ \\
\hline$\overline{(0,0.1)}$ & 0,250 & $0.5,300$ & $0.5,150$ & 0,250 & $0.5,300$ & $0.5,200$ \\
\hline$(0.1,100)$ & $1.5,100$ & $1.5,200$ & $1.5,150$ & $1.5,150$ & $1.5,300$ & $1.5,200$ \\
\hline
\end{tabular}

background contribution is from $p p$ collision processes with high $p_{\mathrm{T}}^{\mathrm{miss}}$, which have the same timing distribution as low- $p_{\mathrm{T}}^{\text {miss }}$ collider data, ensuring that the two discriminating variables are independent for background processes. This includes proton collisions from satellite bunches spaced $\sim 2.5 \mathrm{~ns}$ apart from the main bunches. The noncollision backgrounds, which include cosmic ray muons, beam halo muons, and electronic noise deposits, are reduced to a negligible level by the jet multiplicity requirement and the photon selections.

As the $p_{\mathrm{T}}^{\text {miss }}$ and $t_{\gamma}$ observables are statistically independent for background processes, the background distribution can be factorized into the product of the distributions of these two observables. This permits the use of the so called "ABCD" method to predict the background yield in the signal-enriched bin $\mathrm{C}$ as $N_{\mathrm{C}}=\left(N_{\mathrm{D}} N_{\mathrm{B}}\right) / N_{\mathrm{A}}$, where $N_{\mathrm{X}}$ is the number of background events. In order to account for potential signal contamination in bins $\mathrm{A}, \mathrm{B}$, and $\mathrm{D}$, a modified ABCD method is used where a binned maximum likelihood fit is performed simultaneously in the four bins, with the signal strength included as a floating parameter that scales the signal yield uniformly in each bin. The background component of the fit is constrained to obey the standard ABCD relationship, within the bounds of a small systematic uncertainty derived from a validation check of the method in a control region (CR). Systematic uncertainties that impact the signal and background yields are treated as nuisance parameters with log-normal probability density functions.

For each point in the signal model parameter space $(\Lambda$ and $c \tau$ in Table II), the boundaries in $p_{\mathrm{T}}^{\text {miss }}$ and $t_{\gamma}$ that define the A, B, C, and D bins are chosen to yield optimal expected sensitivity. For the optimization procedure, in order to remain unbiased by the observed data in the signalenriched regions, we estimate the background yields using only the observed yield in data for bin $\mathrm{A}\left(N_{\mathrm{A}}\right)$ as follows. Template shapes for the observable $p_{\mathrm{T}}^{\text {miss }}\left(t_{\gamma}\right)$ are derived from data requiring that $\left|t_{\gamma}\right|<1 \mathrm{~ns}\left(p_{\mathrm{T}}^{\text {miss }}<100 \mathrm{GeV}\right)$. These regions are defined to have negligible signal yield. We obtain the ratios $r_{\mathrm{B} / \mathrm{A}}\left(r_{\mathrm{D} / \mathrm{A}}\right)$ by dividing the number of events with $p_{\mathrm{T}}^{\text {miss }}\left(\left|t_{\gamma}\right|\right)$ larger than the given bin boundary by the number of events with $p_{\mathrm{T}}^{\text {miss }}\left(\left|t_{\gamma}\right|\right)$ smaller than the bin boundary. The background yields in bins $\mathrm{B}, \mathrm{D}$, and $\mathrm{C}$ are calculated as $N_{\mathrm{A}} r_{\mathrm{B} / \mathrm{A}}, N_{\mathrm{A}} r_{\mathrm{D} / \mathrm{A}}$, and $N_{\mathrm{A}} r_{\mathrm{B} / \mathrm{A}} r_{\mathrm{D} / \mathrm{A}}$, respectively. The resulting optimized bin boundaries in $t_{\gamma}$ and $p_{\mathrm{T}}^{\text {miss }}$ are obtained by choosing the bin boundaries that yield the best expected limit and are summarized in Table II for all the SPS8 model parameter space points considered. To simplify the analysis, groups of similar signal model parameters share the same optimized bin boundaries.

It should be noted that we set the lower and upper boundaries in $t_{\gamma}$ to be $-2 \mathrm{~ns}$ and $25 \mathrm{~ns}$, respectively. The lower boundary is set by five times the single photon candidate time resolution, while the upper boundary is set to avoid contamination from the next LHC bunch crossing.

To verify that the $p_{\mathrm{T}}^{\text {miss }}$ and $t_{\gamma}$ observables are independent, we define CRs that isolate different SM processes that are similar to the backgrounds expected in the signal region (SR). The $\gamma+$ jets CR, dominated by the $\gamma+$ jets process, is defined as events satisfying the same requirements as the SR, but having fewer than three jets. The multijet CR, dominated by QCD multijet production, comprises events satisfying the same requirements as the SR, but with an inverted isolation requirement on the leading photon. We measure the correlation coefficients between $p_{\mathrm{T}}^{\text {miss }}$ and $t_{\gamma}$ to be less than $1 \%$ for both the $\gamma+$ jets CR and multijet CR, supporting their independence. A closure test on the predicted background yield in these CRs is propagated as a systematic uncertainty, as discussed further in Sec. VI.

\section{SYSTEMATIC UNCERTAINTIES}

The dominant uncertainty in the search is the statistical uncertainty in the background prediction of the modified ABCD method. There are several subdominant systematic uncertainties that affect the prediction of the signal yield in all four bins. These systematic uncertainties include the uncertainty in the integrated luminosity measurement [50,51], in the energy scale and resolution of the photons and jets, and in the trigger and photon identification efficiencies. For all these cases, dedicated measurements are performed that evaluate corrections and uncertainties in the efficiencies and energy scales in simulated signal events, and these uncertainties are propagated to the signal yield predictions as an uncertainty in the predicted shapes of the distributions of the discriminating observables $p_{\mathrm{T}}^{\text {miss }}$ and $t_{\gamma}$. The calibration of the timestamp discussed in Sec. IV C has associated uncertainties that affect both the offset and the resolution in $t_{\gamma}$, and are propagated in the shape prediction for the $t_{\gamma}$ distribution for the signal 
TABLE III. Summary of systematic uncertainties in the analysis. Also included are notes on whether each source affects signal yields (Sig) or background (Bkg) estimates, to which bins each uncertainty applies, and how the correlations of the uncertainties between the different data sets are treated. We assign different values for the uncertainty in the closure of the background prediction for short and long lifetime signal models. The column labeled 2017 includes both the $2017 \gamma$ and $2017 \gamma \gamma$ categories.

\begin{tabular}{|c|c|c|c|c|c|}
\hline Systematic uncertainty & $\mathrm{Sig} / \mathrm{Bkg}$ & Bins & 2016 & 2017 & Correlation \\
\hline Integrated luminosity & Sig & $\mathrm{A}, \mathrm{B}, \mathrm{C}, \mathrm{D}$ & $2.5 \%$ & $2.3 \%$ & Uncorrelated \\
\hline Photon energy scale & Sig & $\mathrm{A}, \mathrm{B}, \mathrm{C}, \mathrm{D}$ & $1 \%$ & $2 \%$ & Correlated \\
\hline Photon energy resolution & Sig & $\mathrm{A}, \mathrm{B}, \mathrm{C}, \mathrm{D}$ & $1 \%$ & $1 \%$ & Correlated \\
\hline Jet energy scale & Sig & $\mathrm{A}, \mathrm{B}, \mathrm{C}, \mathrm{D}$ & $1.5 \%$ & $2 \%$ & Correlated \\
\hline Jet energy resolution & Sig & A,B,C,D & $1.5 \%$ & $1.5 \%$ & Uncorrelated \\
\hline Photon time bias & Sig & $\mathrm{A}, \mathrm{B}, \mathrm{C}, \mathrm{D}$ & $1.5 \%$ & $1 \%$ & Correlated \\
\hline Photon time resolution & Sig & $\mathrm{A}, \mathrm{B}, \mathrm{C}, \mathrm{D}$ & $0.5 \%$ & $0.5 \%$ & Correlated \\
\hline Trigger efficiency & Sig & $\mathrm{A}, \mathrm{B}, \mathrm{C}, \mathrm{D}$ & $2 \%$ & $<1 \%$ & Uncorrelated \\
\hline Photon identification & Sig & $\mathrm{A}, \mathrm{B}, \mathrm{C}, \mathrm{D}$ & $2 \%$ & $3 \%$ & Correlated \\
\hline Closure in bin $\mathrm{C}(c \tau \leq 0.1 \mathrm{~m})$ & $\mathrm{Bkg}$ & $\mathrm{C}$ & $2 \%$ & $3.5 \%$ & Correlated \\
\hline Closure in bin $\mathrm{C}(c \tau>0.1 \mathrm{~m})$ & $\mathrm{Bkg}$ & $\mathrm{C}$ & $90 \%$ & $90 \%$ & Correlated \\
\hline
\end{tabular}

benchmarks. As we use $Z \rightarrow e^{+} e^{-}$events to measure the photon identification efficiency, the corresponding systematic uncertainty includes the impact of the difference in detector response between an electron and a photon. Table III provides a summary of the systematic uncertainties in the analysis and their assigned values for each data set, as well as additional information about the correlations between the uncertainties.

As the modified ABCD method for estimating the background requires that the discriminating observables $p_{\mathrm{T}}^{\text {miss }}$ and $t_{\gamma}$ are independent, we propagate a systematic uncertainty for any potential interdependence of these observables. We select events in the $\gamma+$ jets and multijet $\mathrm{CR}$ and separate events into the same A, B, C, and D bins defined for the signal region. We compare the background yield in bin $\mathrm{C}$ predicted by the $\mathrm{ABCD}$ method with the observed yield, and propagate the difference as a systematic uncertainty. This systematic uncertainty is referred to as "the closure" in Table III. For the cases with neutralino proper decay length smaller than $0.1 \mathrm{~m}$, this systematic uncertainty is relatively small, at $4 \%$ or less. For the cases with neutralino proper decay length larger than $0.1 \mathrm{~m}$, the data yields in bin $\mathrm{C}$ of the CRs are small and are limited by statistical uncertainty. As a result, a relatively large systematic uncertainty of $90 \%$ of the predicted background yield is propagated.

\section{RESULTS AND INTERPRETATION}

Tables IV and V list the yields and postfit background predictions for the background-only fit in each of the

TABLE IV. Observed number of events $\left(N_{\mathrm{obs}}^{\text {data }}\right)$ and predicted background yields from the background-only fit $\left(N_{\mathrm{bkg}}^{\text {postfit }}\right)$ in bins $\mathrm{A}, \mathrm{B}, \mathrm{C}$, and $\mathrm{D}$ in data for the 2016 category and for the different $t_{\gamma}$ and $p_{\mathrm{T}}^{\text {miss }}$ bin boundaries summarized in Table II. In addition, the predicted postfit yields from the background-only fit not including bin $\mathrm{C}\left(N_{\mathrm{bkg}(\mathrm{noC})}^{\text {postit }}\right)$ are provided as a test of the closure. Uncertainties in the $N_{\mathrm{bkg}}^{\text {postfit }}$ and $N_{\mathrm{bkg} \text { (no C) }}^{\text {postfit }}$ values are the postfit uncertainties. The propagation of the systematic uncertainties is handled during the fit and therefore they are included in the postfit uncertainties.

\begin{tabular}{|c|c|c|c|c|c|}
\hline \multicolumn{6}{|c|}{2016 category } \\
\hline \multicolumn{2}{|c|}{ Bin boundary $\left[t_{\gamma}(\mathrm{ns}), p_{\mathrm{T}}^{\text {miss }}(\mathrm{GeV})\right]$} & A & $\mathrm{B}$ & $\mathrm{C}$ & $\mathrm{D}$ \\
\hline \multirow[t]{3}{*}{$(0,250)$} & $N_{\text {obs }}^{\text {data }}$ & 16139 & 41 & 62 & 18826 \\
\hline & $N_{\text {bkg }}^{\text {postfit }}$ & $16130 \pm 110$ & $47.5 \pm 4.8$ & $55.6 \pm 5.6$ & $18830 \pm 130$ \\
\hline & $N_{\mathrm{bkg}(\text { no C })}^{\text {postfit }}$ & $16140 \pm 110$ & $41.0 \pm 6.5$ & $47.8 \pm 7.7$ & $18830 \pm 130$ \\
\hline \multirow[t]{3}{*}{$(1.5,100)$} & $N_{\mathrm{obs}}^{\text {data }}$ & 33760 & 1302 & 1 & 5 \\
\hline & $N_{\text {bkg }}^{\text {postfit }}$ & $33760 \pm 160$ & $1303 \pm 37$ & $0.29 \pm 0.28$ & $5.7 \pm 2.2$ \\
\hline & $N_{\mathrm{bkg}(\text { no C })}^{\text {postfit }}$ & $33760 \pm 160$ & $1302 \pm 37$ & $0.19 \pm 0.21$ & $5.0 \pm 2.1$ \\
\hline \multirow[t]{3}{*}{$(1.5,150)$} & $N_{\text {obs }}^{\text {data }}$ & 34595 & 467 & 0 & 6 \\
\hline & $N_{\text {bkg }}^{\text {postfit }}$ & $34600 \pm 170$ & $467 \pm 22$ & $0.08 \pm 0.08$ & $5.9 \pm 2.3$ \\
\hline & $N_{\text {bkg(noC) }}^{\text {postfit }}$ & $34600 \pm 170$ & $467 \pm 22$ & $0.08 \pm 0.09$ & $6.0 \pm 2.3$ \\
\hline
\end{tabular}


TABLE V. Observed number of events $\left(N_{\mathrm{obs}}^{\text {data }}\right)$ and predicted background yields from the background-only fit $\left(N_{\mathrm{bkg}}^{\mathrm{postit}}\right)$ in bins A, B, C, and $\mathrm{D}$ in data for the $2017 \gamma$ (upper table) and $2017 \gamma \gamma$ (lower table) categories and for the different $t_{\gamma}$ and $p_{\mathrm{T}}^{\text {miss }}$ bin boundaries summarized in Table II. Additional details are described in the caption of Table IV.

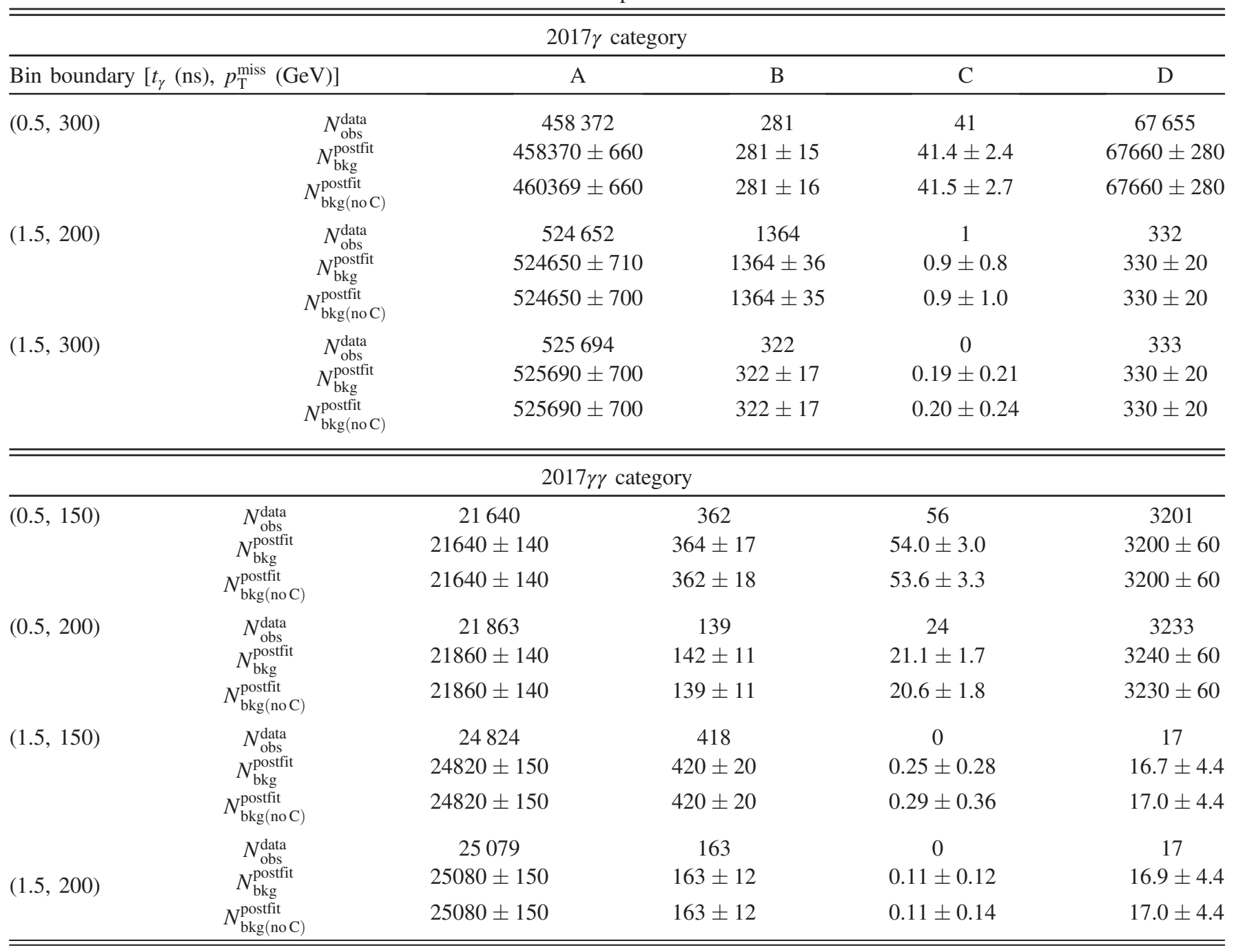

four bins of the 2016, 2017 , and $2017 \gamma \gamma$ categories, respectively, for all the $t_{\gamma}-p_{\mathrm{T}}^{\text {miss }}$ bin boundaries used. No statistically significant deviation from the background expectation is observed. The search result is interpreted in terms of limits on the neutralino production cross section for scenarios in the GMSB SPS8 signal model set.

The modified frequentist criterion $\mathrm{CL}_{\mathrm{s}}$ [52-54] with the profile likelihood ratio test statistic determined by toy experiments is used to evaluate the observed and expected limits at 95\% confidence level (C.L.) on the signal production cross sections. The limits are shown in Fig. 5 as functions of the mass of the neutralino NLSP $\tilde{\chi}_{1}^{0}$ (linearly related to the SUSY breaking scale, $\Lambda$ ) and the proper decay length of the neutralino. The two-photon category (2016 and 2017 $\gamma \gamma$ ) and the one-photon category (2017 $\gamma$ ) are complementary as the sensitivity at small proper decay length is better for the 2016 and $2017 \gamma \gamma$ categories because of the extra background suppression from requiring two photons, while the sensitivity at large proper decay lengths is better for the $2017 \gamma$ analysis because of the significantly improved signal acceptance from the dedicated displaced single-photon trigger. As a result, the sensitivity to signal models with proper lifetimes greater than the ECAL timing resolution for a single photon candidate is improved compared to previous results. For the neutralino proper decay lengths $c \tau$ of $0.1,1,10$, and $100 \mathrm{~m}$, masses up to about $320,525,360$, and $215 \mathrm{GeV}$ are excluded at 95\% C.L., respectively.

\section{SUMMARY}

A search for long-lived particles that decay to a photon and a weakly interacting particle has been presented. The search is based on proton-proton collisions at a 


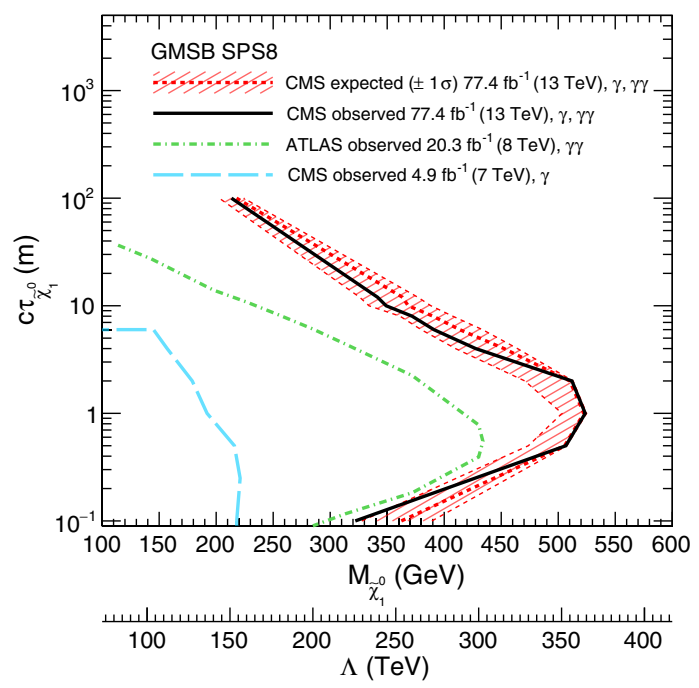

FIG. 5. The $95 \%$ C.L. exclusion contours for the GMSB neutralino production cross section, shown as functions of the neutralino mass, or equivalently the SUSY breaking scale, $\Lambda$, in the GMSB SPS8 model, and the neutralino proper decay length, $c \tau_{\tilde{\chi}_{1}^{0}}$.

center-of-mass energy of $13 \mathrm{TeV}$ collected by the CMS experiment in 2016-2017. The photon from this particle's decay would enter the electromagnetic calorimeter at nonnormal impact angles and with delayed times, and this striking combination of features is exploited to suppress backgrounds. The search is performed using a combination of the 2016 and 2017 data sets, corresponding to a total integrated luminosity of $77.4 \mathrm{fb}^{-1}$. Both single-photon and diphoton event samples are used for the search, with each sample providing a complementary sensitivity at larger and smaller long-lived particle proper decay lengths, respectively. The results are interpreted in the context of supersymmetry with gauge-mediated supersymmetry breaking, using the SPS8 benchmark model. For neutralino proper decay lengths of $0.1,1,10$, and $100 \mathrm{~m}$, masses up to about $320,525,360$, and $215 \mathrm{GeV}$ are excluded at 95\% confidence level, respectively. The previous best limits are extended by one order of magnitude in the neutralino proper decay length and by $100 \mathrm{GeV}$ in the mass reach.

\section{ACKNOWLEDGMENTS}

We congratulate our colleagues in the CERN accelerator departments for the excellent performance of the LHC and thank the technical and administrative staffs at CERN and at other CMS institutes for their contributions to the success of the CMS effort. In addition, we gratefully acknowledge the computing centers and personnel of the Worldwide LHC Computing Grid for delivering so effectively the computing infrastructure essential to our analyses. Finally, we acknowledge the enduring support for the construction and operation of the LHC and the CMS detector provided by the following funding agencies: BMBWF and FWF (Austria); FNRS and FWO (Belgium); CNPq, CAPES, FAPERJ, FAPERGS, and FAPESP (Brazil); MES (Bulgaria); CERN; CAS, MoST, and NSFC (China); COLCIENCIAS (Colombia); MSES and CSF (Croatia); RPF (Cyprus); SENESCYT (Ecuador); MoER, ERC IUT, PUT and ERDF (Estonia); Academy of Finland, MEC, and HIP (Finland); CEA and CNRS/IN2P3 (France); BMBF, DFG, and HGF (Germany); GSRT (Greece); NKFIA (Hungary); DAE and DST (India); IPM (Iran); SFI (Ireland); INFN (Italy); MSIP and NRF (Republic of Korea); MES (Latvia); LAS (Lithuania); MOE and UM (Malaysia); BUAP, CINVESTAV, CONACYT, LNS, SEP, and UASLP-FAI (Mexico); MOS (Montenegro); MBIE (New Zealand); PAEC (Pakistan); MSHE and NSC (Poland); FCT (Portugal); JINR (Dubna); MON, RosAtom, RAS, RFBR, and NRC KI (Russia); MESTD (Serbia); SEIDI, CPAN, PCTI, and FEDER (Spain); MOSTR (Sri Lanka); Swiss Funding Agencies (Switzerland); MST (Taipei); ThEPCenter, IPST, STAR, and NSTDA (Thailand); TUBITAK and TAEK (Turkey); NASU and SFFR (Ukraine); STFC (United Kingdom); DOE and NSF (USA). Individuals have received support from the Marie-Curie program and the European Research Council and Horizon 2020 Grant, contract Nos. 675440, 752730, and 765710 (European Union); the Leventis Foundation; the A.P. Sloan Foundation; the Alexander von Humboldt Foundation; the Belgian Federal Science Policy Office; the Fonds pour la Formation à la Recherche dans l'Industrie et dans l'Agriculture (FRIA-Belgium); the Agentschap voor Innovatie door Wetenschap en Technologie (IWT-Belgium); the F. R. S.-FNRS and FWO (Belgium) under the "Excellence of Science-EOS"-be.h project n. 30820817; the Beijing Municipal Science \& Technology Commission, No. Z181100004218003; the Ministry of Education, Youth and Sports (MEYS) of the Czech Republic; the Lendület ("Momentum") Program and the János Bolyai Research Scholarship of the Hungarian Academy of Sciences, the New National Excellence Program ÚNKP, the NKFIA research grants No. 123842, No. 123959 , No. 124845 , No. 124850 , No. 125105, No. 128713, No. 128786, and No. 129058 (Hungary); the Council of Science and Industrial Research, India; the HOMING PLUS program of the Foundation for Polish Science, cofinanced from European Union, Regional Development Fund, the Mobility Plus program of the Ministry of Science and Higher Education, the National Science Center (Poland), contracts Harmonia 2014/14/M/ ST2/00428, Opus 2014/13/B/ST2/02543, 2014/15/B/ST2/ 03998, and 2015/19/B/ST2/02861, Sonata-bis 2012/07/E/ ST2/01406; the National Priorities Research Program by Qatar National Research Fund; the Ministry of Science and Education, grant no. 3.2989.2017 (Russia); the Programa Estatal de Fomento de la Investigación Científica y Técnica de Excelencia María de Maeztu, grant MDM-2015-0509 
and the Programa Severo Ochoa del Principado de Asturias; the Thalis and Aristeia programs cofinanced by EU-ESF and the Greek NSRF; the Rachadapisek Sompot Fund for Postdoctoral Fellowship, Chulalongkorn University and the Chulalongkorn Academic into Its 2nd Century Project Advancement Project (Thailand); the Nvidia Corporation; the Welch Foundation, contract C-1845; and the Weston Havens Foundation (USA).
[1] P. Ramond, Dual theory for free fermions, Phys. Rev. D 3, 2415 (1971).

[2] P. Ramond, An interpretation of dual theories, Nuovo Cimento A 4, 544 (1971).

[3] Y. A. Gol'fand and E. P. Likhtman, Extension of the algebra of Poincaré group generators and violation of $P$ invariance, JETP Lett. 13, 323 (1971).

[4] D. V. Volkov and V. P. Akulov, Possible universal neutrino interaction, JETP Lett. 16, 438 (1972).

[5] J. Wess and B. Zumino, Supergauge transformations in fourdimensions, Nucl. Phys. B70, 39 (1974).

[6] D. Z. Freedman, P. van Nieuwenhuizen, and S. Ferrara, Progress toward a theory of supergravity, Phys. Rev. D 13, 3214 (1976).

[7] S. Deser and B. Zumino, Consistent supergravity, Phys. Lett. 62B, 335 (1976).

[8] D. Z. Freedman and P. van Nieuwenhuizen, Properties of supergravity theory, Phys. Rev. D 14, 912 (1976).

[9] S. Ferrara and P. van Nieuwenhuizen, Consistent Supergravity with Complex Spin 3/2 Gauge Fields, Phys. Rev. Lett. 37, 1669 (1976).

[10] P. Fayet, Supergauge invariant extension of the Higgs mechanism and a model for the electron and its neutrino, Nucl. Phys. B90, 104 (1975).

[11] A. H. Chamseddine, R. L. Arnowitt, and P. Nath, Locally Supersymmetric Grand Unification, Phys. Rev. Lett. 49, 970 (1982).

[12] R. Barbieri, S. Ferrara, and C. A. Savoy, Gauge models with spontaneously broken local supersymmetry, Phys. Lett. 119B, 343 (1982).

[13] L. J. Hall, J. D. Lykken, and S. Weinberg, Supergravity as the messenger of supersymmetry breaking, Phys. Rev. D 27, 2359 (1983).

[14] G. L. Kane, C. F. Kolda, L. Roszkowski, and J. D. Wells, Study of constrained minimal supersymmetry, Phys. Rev. D 49, 6173 (1994).

[15] G. F. Giudice and R. Rattazzi, Theories with gauge mediated supersymmetry breaking, Phys. Rep. 322, 419 (1999).

[16] S. Dimopoulos, M. Dine, S. Raby, and S. D. Thomas, Experimental Signatures of Low-Energy Gauge Mediated Supersymmetry Breaking, Phys. Rev. Lett. 76, 3494 (1996).

[17] P. Fayet, Mixing between gravitational and weak interactions through the massive gravitino, Phys. Lett. B 70, 461 (1977).

[18] H. Baer, M. Brhlik, C. H. Chen, and X. Tata, Signals for the minimal gauge-mediated supersymmetry breaking model at the Fermilab Tevatron collider, Phys. Rev. D 55, 4463 (1997).
[19] H. Baer, P. G. Mercadante, X. Tata, and Y. L Wang, Reach of Tevatron upgrades in gauge-mediated supersymmetry breaking models, Phys. Rev. D 60, 055001 (1999).

[20] S. Dimopoulos, S. Thomas, and J. D. Wells, Sparticle spectroscopy and electroweak symmetry breaking with gauge-mediated supersymmetry breaking, Nucl. Phys. B488, 39 (1997).

[21] J. R. Ellis, J. L. Lopez, and D. V. Nanopoulos, Analysis of LEP constraints on supersymmetric models with a light gravitino, Phys. Lett. B 394, 354 (1997).

[22] M. Dine, A. E. Nelson, Y. Nir, and Y. Shirman, New tools for low energy dynamical supersymmetry breaking, Phys. Rev. D 53, 2658 (1996).

[23] G. F. Giudice and R. Rattazzi, Gauge-mediated supersymmetry breaking, in Perspectives on Supersymmetry (World Scientific, Singapore, 1998), p. 355.

[24] B. C. Allanach et al., The Snowmass points and slopes: Benchmarks for SUSY searches, Eur. Phys. J. C 25, 113 (2002).

[25] C. H. Chen and J. F. Gunion, Maximizing hadron collider sensitivity to gauge mediated supersymmetry breaking models, Phys. Lett. B 420, 77 (1998).

[26] CMS Collaboration, Search for long-lived particles decaying to photons and missing energy in protonproton collisions at $\sqrt{s}=7 \mathrm{TeV}$, Phys. Lett. B 722, 273 (2013).

[27] ATLAS Collaboration, Search for nonpointing and delayed photons in the diphoton and missing transverse momentum final state in $8 \mathrm{TeV} p p$ collisions at the LHC using the ATLAS detector, Phys. Rev. D 90, 112005 (2014).

[28] CMS Collaboration, Search for exotic decays of a Higgs boson into undetectable particles and one or more photons, Phys. Lett. B 753, 363 (2016).

[29] CMS Collaboration, The CMS experiment at the CERN LHC, J. Instrum. 3, S08004 (2008).

[30] CMS Collaboration, The CMS trigger system, J. Instrum. 12, P01020 (2017).

[31] J. Alwall, S. Höche, F. Krauss, N. Lavesson, L. Lönnblad, F. Maltoni, M. L. Mangano, M. Moretti, C. G. Papadopoulos, F. Piccinini, S. Schumann, M. Treccani, J. Winter, and M. Worek, Comparative study of various algorithms for the merging of parton showers and matrix elements in hadronic collisions, Eur. Phys. J. C 53, 473 (2008).

[32] E. Bothmann et al., Event generation with SHERPA 2.2, SciPost Phys. 7, 034 (2019).

[33] T. Gleisberg, S. Hoeche, F. Krauss, M. Schonherr, S. Schumann, F. Siegert, and J. Winter, Event generation with SHERPA 1.1, J. High Energy Phys. 02 (2009) 007. 
[34] F. E. Paige, S. D. Protopopescu, H. Baer, and X. Tata, ISAJET 7.69: A Monte Carlo event generator for $p p$, $\bar{p} p$, and $e^{+} e^{-}$reactions, arXiv:hep-ph/0312045.

[35] T. Sjöstrand, S. Ask, J. R. Christiansen, R. Corke, N. Desai, P. Ilten, S. Mrenna, S. Prestel, C. O. Rasmussen, and P. Z. Skands, An introduction to PYTHIA 8.2, Comput. Phys. Commun. 191, 159 (2015).

[36] P. Skands, S. Carrazza, and J. Rojo, Tuning PYTHIA 8.1: The Monash 2013 tune, Eur. Phys. J. C 74, 3024 (2014).

[37] CMS Collaboration, Underlying event tunes and double parton scattering, CMS Physics Analysis Summary Report No. CMS-PAS-GEN-14-001, 2018, http://cds.cern.ch/record/ 1697700.

[38] CMS Collaboration, Extraction and validation of a new set of CMS PYTHIA8 tunes from underlying-event measurements, arXiv:1903.12179 [Eur. Phys. J. C (to be published)].

[39] R. D. Ball et al. (NNPDF Collaboration), Parton distributions for the LHC Run II, J. High Energy Phys. 04 (2015) 040.

[40] R. D. Ball et al. (NNPDF Collaboration), Parton distributions from high-precision collider data, Eur. Phys. J. C 77, 663 (2017).

[41] S. Agostinelli et al. (GEANT4 Collaboration), GEANT4-a simulation toolkit, Nucl. Instrum. Methods Phys. Res., Sect. A 506, 250 (2003).

[42] CMS Collaboration, Particle-flow reconstruction and global event description with the CMS detector, J. Instrum. 12, P10003 (2017).

[43] M. Cacciari, G. P. Salam, and G. Soyez, The anti- $k_{t}$ jet clustering algorithm, J. High Energy Phys. 04 (2008) 063.

[44] M. Cacciari, G. P. Salam, and G. Soyez, FastJet user manual, Eur. Phys. J. C 72, 1896 (2012).
[45] CMS Collaboration, Performance of photon reconstruction and identification with the CMS detector in proton-proton collisions at $\sqrt{s}=8 \mathrm{TeV}$, J. Instrum. 10, P08010 (2015).

[46] CMS Collaboration, Jet algorithms performance in $13 \mathrm{TeV}$ data, CMS Physics Analysis Summary Report No. CMS-PASJME-16-003, 2017, https://cds.cern.ch/record/2256875.

[47] CMS Collaboration, Performance of missing transverse momentum reconstruction in proton-proton collisions at $\sqrt{\mathrm{s}}=$ 13 TeV using the CMS detector, J. Instrum. 14, P07004 (2019).

[48] CMS Collaboration, Time reconstruction and performance of the CMS electromagnetic calorimeter, J. Instrum. 5, T03011 (2010).

[49] D. del Re, Timing performance of the CMS ECAL and prospects for the future, J. Phys. 587, 012003 (2015).

[50] CMS Collaboration, CMS Luminosity Measurements for the 2016 data-taking period, CMS Physics Analysis Summary Report No. CMS-PAS-LUM-17-001, 2017, https://cds .cern.ch/record/2257069.

[51] CMS Collaboration, CMS luminosity measurement for the 2017 data-taking period at $\sqrt{s}=13 \mathrm{TeV}$, CMS Physics Analysis Summary Report No. CMS-PAS-LUM-17-004, 2018, https://cds.cern.ch/record/2621960.

[52] T. Junk, Confidence level computation for combining searches with small statistics, Nucl. Instrum. Methods Phys. Res., Sect. A 434, 435 (1999).

[53] A. L. Read, Presentation of search results: The $C L_{s}$ technique, J. Phys. G 28, 2693 (2002).

[54] The ATLAS Collaboration, The CMS Collaboration, The LHC higgs combination group, Procedure for the LHC Higgs Boson Search Combination in Summer 2011, CMS Note, (Tech. Rep. CMS-NOTE-2011-005, ATL-PHYSPUB-2011-11, 2011).

A. M. Sirunyan, ${ }^{1, a}$ A. Tumasyan, ${ }^{1}$ W. Adam, ${ }^{2}$ F. Ambrogi, ${ }^{2}$ T. Bergauer, ${ }^{2}$ J. Brandstetter, ${ }^{2}$ M. Dragicevic, ${ }^{2}$ J. Erö, ${ }^{2}$ A. Escalante Del Valle, ${ }^{2}$ M. Flechl, ${ }^{2}$ R. Frühwirth, ${ }^{2, b}$ M. Jeitler, ${ }^{2, b}$ N. Krammer, ${ }^{2}$ I. Krätschmer, ${ }^{2}$ D. Liko, ${ }^{2}$ T. Madlener, ${ }^{2}$ I. Mikulec, ${ }^{2}$ N. Rad, ${ }^{2}$ J. Schieck, ${ }^{2, b}$ R. Schöfbeck, ${ }^{2}$ M. Spanring, ${ }^{2}$ D. Spitzbart, ${ }^{2}$ W. Waltenberger, ${ }^{2}$ C.-E. Wulz, ${ }^{2, b}$ M. Zarucki, ${ }^{2}$ V. Drugakov, ${ }^{3}$ V. Mossolov, ${ }^{3}$ J. Suarez Gonzalez, ${ }^{3}$ M. R. Darwish, ${ }^{4}$ E. A. De Wolf, ${ }^{4}$ D. Di Croce, ${ }^{4}$ X. Janssen, ${ }^{4}$ A. Lelek, ${ }^{4}$ M. Pieters, ${ }^{4}$ H. Rejeb Sfar, ${ }^{4}$ H. Van Haevermaet, ${ }^{4}$ P. Van Mechelen, ${ }^{4}$ S. Van Putte, ${ }^{4}$ N. Van Remortel, ${ }^{4}$ F. Blekman, ${ }^{5}$ E. S. Bols, ${ }^{5}$ S. S. Chhibra, ${ }^{5}$ J. D'Hondt, ${ }^{5}$ J. De Clercq,${ }^{5}$ D. Lontkovskyi, ${ }^{5}$ S. Lowette, ${ }^{5}$ I. Marchesini, ${ }^{5}$ S. Moortgat, ${ }^{5}$ Q. Python, ${ }^{5}$ K. Skovpen, ${ }^{5}$ S. Tavernier, ${ }^{5}$ W. Van Doninck, ${ }^{5}$ P. Van Mulders, ${ }^{5}$ D. Beghin, ${ }^{6}$ B. Bilin, ${ }^{6}$ H. Brun, ${ }^{6}$ B. Clerbaux, ${ }^{6}$ G. De Lentdecker, ${ }^{6}$ H. Delannoy, ${ }^{6}$ B. Dorney, ${ }^{6}$ L. Favart, ${ }^{6}$ A. Grebenyuk, ${ }^{6}$ A. K. Kalsi, ${ }^{6}$ A. Popov, ${ }^{6}$ N. Postiau, ${ }^{6}$ E. Starling, ${ }^{6}$ L. Thomas, ${ }^{6}$ C. Vander Velde, ${ }^{6}$ P. Vanlaer, ${ }^{6}$ D. Vannerom, ${ }^{6}$ T. Cornelis, ${ }^{7}$ D. Dobur, ${ }^{7}$ I. Khvastunov, ${ }^{7, \mathrm{c}}$ M. Niedziela, ${ }^{7}$ C. Roskas, ${ }^{7}$ D. Trocino, ${ }^{7}$ M. Tytgat, ${ }^{7}$ W. Verbeke, ${ }^{7}$ B. Vermassen, ${ }^{7}$ M. Vit, ${ }^{7}$ O. Bondu, ${ }^{8}$ G. Bruno, ${ }^{8}$ C. Caputo, ${ }^{8}$ P. David,${ }^{8}$ C. Delaere,${ }^{8}$ M. Delcourt, ${ }^{8}$ A. Giammanco, ${ }^{8}$ V. Lemaitre, ${ }^{8}$ J. Prisciandaro, ${ }^{8}$ A. Saggio, ${ }^{8}$ M. Vidal Marono, ${ }^{8}$ P. Vischia, ${ }^{8}$ J. Zobec ${ }^{8}$ F. L. Alves, ${ }^{9}$ G. A. Alves, ${ }^{9}$ G. Correia Silva, ${ }^{9}$ C. Hensel, ${ }^{9}$ A. Moraes, ${ }^{9}$ P. Rebello Teles, ${ }^{9}$ E. Belchior Batista Das Chagas, ${ }^{10}$ W. Carvalho, ${ }^{10}$ J. Chinellato, ${ }^{10, d}$ E. Coelho, ${ }^{10}$ E. M. Da Costa, ${ }^{10}$ G. G. Da Silveira, ${ }^{10, e}$ D. De Jesus Damiao, ${ }^{10}$ C. De Oliveira Martins, ${ }^{10}$ S. Fonseca De Souza, ${ }^{10}$ L. M. Huertas Guativa, ${ }^{10}$ H. Malbouisson, ${ }^{10}$ J. Martins, ${ }^{10, f}$ D. Matos Figueiredo, ${ }^{10}$ M. Medina Jaime ${ }^{10, g}$ M. Melo De Almeida, ${ }^{10}$ C. Mora Herrera, ${ }^{10}$ L. Mundim, ${ }^{10}$ H. Nogima, ${ }^{10}$ W. L. Prado Da Silva, ${ }^{10}$ L. J. Sanchez Rosas, ${ }^{10}$ A. Santoro, ${ }^{10}$ A. Sznajder, ${ }^{10}$ M. Thiel, ${ }^{10}$ E. J. Tonelli Manganote, ${ }^{10, d}$ F. Torres Da Silva De Araujo, ${ }^{10}$ A. Vilela Pereira, ${ }^{10}$ C. A. Bernardes, ${ }^{11 a}$ L. Calligaris, ${ }^{11 a}$ T. R. Fernandez Perez Tomei, ${ }^{11 \mathrm{a}}$ E. M. Gregores, ${ }^{11 \mathrm{a}, 1 \mathrm{~b}}$ D. S. Lemos, ${ }^{11 \mathrm{a}}$ P. G. Mercadante, ${ }^{11 \mathrm{a}, 1 \mathrm{~b}}$ S. F. Novaes, ${ }^{11 \mathrm{a}}$ Sandra S. Padula, ${ }^{11 a}$ A. Aleksandrov, ${ }^{12}$ G. Antchev, ${ }^{12}$ R. Hadjiiska, ${ }^{12}$ P. Iaydjiev, ${ }^{12}$ M. Misheva, ${ }^{12}$ M. Rodozov, ${ }^{12}$ M. Shopova, ${ }^{12}$ G. Sultanov, ${ }^{12}$ M. Bonchev, ${ }^{13}$ A. Dimitrov, ${ }^{13}$ T. Ivanov, ${ }^{13}$ L. Litov, ${ }^{13}$ B. Pavlov, ${ }^{13}$ P. Petkov, ${ }^{13}$ W. Fang, ${ }^{14, h}$ 
X. Gao, ${ }^{14, h}$ L. Yuan, ${ }^{14}$ G. M. Chen, ${ }^{15}$ H. S. Chen, ${ }^{15}$ M. Chen, ${ }^{15}$ C. H. Jiang, ${ }^{15}$ D. Leggat, ${ }^{15}$ H. Liao, ${ }^{15}$ Z. Liu, ${ }^{15}$ A. Spiezia, ${ }^{15}$ J. Tao, ${ }^{15}$ E. Yazgan, ${ }^{15}$ H. Zhang, ${ }^{15}$ S. Zhang, ${ }^{15, i}$ J. Zhao, ${ }^{15}$ A. Agapitos, ${ }^{16}$ Y. Ban, ${ }^{16}$ G. Chen, ${ }^{16}$ A. Levin, ${ }^{16} \mathrm{~J} . \mathrm{Li},{ }^{16} \mathrm{~L} . \mathrm{Li},{ }^{16}$ Q. Li ${ }^{16}$ Y. Mao, ${ }^{16}$ S. J. Qian, ${ }^{16}$ D. Wang, ${ }^{16}$ Q. Wang, ${ }^{16}$ M. Ahmad, ${ }^{17}$ Z. Hu, ${ }^{17}$ Y. Wang, ${ }^{17}$ M. Xiao, ${ }^{18}$ C. Avila, ${ }^{19}$ A. Cabrera ${ }^{19}$ C. Florez, ${ }^{19}$ C. F. González Hernández, ${ }^{19}$ M. A. Segura Delgado, ${ }^{19}$ J. Mejia Guisao, ${ }^{20}$ J. D. Ruiz Alvarez, ${ }^{20}$ C. A. Salazar González, ${ }^{20}$ N. Vanegas Arbelaez, ${ }^{20}$ D. Giljanović, ${ }^{21}$ N. Godinovic, ${ }^{21}$ D. Lelas, ${ }^{21}$ I. Puljak, ${ }^{21}$ T. Sculac, ${ }^{21}$ Z. Antunovic, ${ }^{22}$ M. Kovac, ${ }^{22}$ V. Brigljevic, ${ }^{23}$ D. Ferencek, ${ }^{23}$ K. Kadija ${ }^{23}$ B. Mesic, ${ }^{23}$ M. Roguljic, ${ }^{23}$ A. Starodumov, ${ }^{23, j}$ T. Susa, ${ }^{23}$ M. W. Ather, ${ }^{24}$ A. Attikis, ${ }^{24}$ E. Erodotou, ${ }^{24}$ A. Ioannou, ${ }^{24}$ M. Kolosova, ${ }^{24}$ S. Konstantinou, ${ }^{24}$

G. Mavromanolakis, ${ }^{24}$ J. Mousa, ${ }^{24}$ C. Nicolaou, ${ }^{24}$ F. Ptochos,${ }^{24}$ P. A. Razis, ${ }^{24}$ H. Rykaczewski, ${ }^{24}$ D. Tsiakkouri, ${ }^{24}$ M. Finger, ${ }^{25, k}$ M. Finger Jr., ${ }^{25, k}$ A. Kveton, ${ }^{25}$ J. Tomsa, ${ }^{25}$ E. Ayala, ${ }^{26}$ E. Carrera Jarrin, ${ }^{27}$ Y. Assran, ${ }^{28,1, m}$ S. Elgammal, ${ }^{28,1}$ S. Bhowmik, ${ }^{29}$ A. Carvalho Antunes De Oliveira, ${ }^{29}$ R. K. Dewanjee, ${ }^{29}$ K. Ehataht, ${ }^{29}$ M. Kadastik,${ }^{29}$ M. Raidal, ${ }^{29}$ C. Veelken, ${ }^{29}$ P. Eerola ${ }^{30}$ L. Forthomme, ${ }^{30}$ H. Kirschenmann, ${ }^{30}$ K. Osterberg, ${ }^{30}$ M. Voutilainen, ${ }^{30}$ F. Garcia, ${ }^{31}$ J. Havukainen, ${ }^{31}$ J. K. Heikkilä, ${ }^{31}$ V. Karimäki, ${ }^{31}$ M. S. Kim, ${ }^{31}$ R. Kinnunen, ${ }^{31}$ T. Lampén, ${ }^{31}$ K. Lassila-Perini, ${ }^{31}$ S. Laurila, ${ }^{31}$ S. Lehti, ${ }^{31}$ T. Lindén, ${ }^{31}$ P. Luukka, ${ }^{31}$ T. Mäenpää, ${ }^{31}$ H. Siikonen, ${ }^{31}$ E. Tuominen, ${ }^{31}$ J. Tuominiemi,${ }^{31}$ T. Tuuva, ${ }^{32}$ M. Besancon, ${ }^{33}$ F. Couderc, ${ }^{33}$ M. Dejardin, ${ }^{33}$ D. Denegri, ${ }^{33}$ B. Fabbro, ${ }^{33}$ J. L. Faure, ${ }^{33}$ F. Ferri, ${ }^{33}$ S. Ganjour, ${ }^{33}$ A. Givernaud, ${ }^{33}$ P. Gras, ${ }^{33}$ G. Hamel de Monchenault, ${ }^{33}$ P. Jarry, ${ }^{33}$ C. Leloup,${ }^{33}$ E. Locci, ${ }^{33}$ J. Malcles, ${ }^{33}$ J. Rander, ${ }^{33}$ A. Rosowsky, ${ }^{33}$ M. Ö. Sahin, ${ }^{33}$ A. Savoy-Navarro, ${ }^{33, n}$ M. Titov, ${ }^{33}$ S. Ahuja,${ }^{34}$ C. Amendola,${ }^{34}$ F. Beaudette,${ }^{34}$ P. Busson, ${ }^{34}$ C. Charlot, ${ }^{34}$ B. Diab, ${ }^{34}$ G. Falmagne,${ }^{34}$ R. Granier de Cassagnac, ${ }^{34}$ I. Kucher, ${ }^{34}$ A. Lobanov, ${ }^{34}$ C. Martin Perez,${ }^{34}$ M. Nguyen, ${ }^{34}$ C. Ochando, ${ }^{34}$ P. Paganini, ${ }^{34}$ J. Rembser, ${ }^{34}$ R. Salerno, ${ }^{34}$ J. B. Sauvan, ${ }^{34}$ Y. Sirois, ${ }^{34}$ A. Zabi,${ }^{34}$ A. Zghiche, ${ }^{34}$ J.-L. Agram, ${ }^{35,0}$ J. Andrea ${ }^{35}$ D. Bloch,${ }^{35}$ G. Bourgatte, ${ }^{35}$ J.-M. Brom,${ }^{35}$ E. C. Chabert, ${ }^{35}$ C. Collard,${ }^{35}$ E. Conte,,${ }^{35,0}$ J.-C. Fontaine, ${ }^{35,0}$ D. Gelé, ${ }^{35}$ U. Goerlach, ${ }^{35}$ M. Jansová, ${ }^{35}$ A.-C. Le Bihan, ${ }^{35}$ N. Tonon, ${ }^{35}$ P. Van Hove,${ }^{35}$ S. Gadrat,${ }^{36}$ S. Beauceron, ${ }^{37}$ C. Bernet,${ }^{37}$ G. Boudoul,${ }^{37}$ C. Camen, ${ }^{37}$ A. Carle,${ }^{37}$ N. Chanon, ${ }^{37}$ R. Chierici,${ }^{37}$ D. Contardo, ${ }^{37}$ P. Depasse, ${ }^{37}$ H. El Mamouni, ${ }^{37}$ J. Fay, ${ }^{37}$ S. Gascon, ${ }^{37}$ M. Gouzevitch, ${ }^{37}$ B. Ille, ${ }^{37}$ Sa. Jain, ${ }^{37}$ F. Lagarde, ${ }^{37}$ I. B. Laktineh, ${ }^{37}$ H. Lattaud,${ }^{37}$ A. Lesauvage, ${ }^{37}$ M. Lethuillier, ${ }^{37}$ L. Mirabito, ${ }^{37}$ S. Perries,${ }^{37}$ V. Sordini,${ }^{37}$ L. Torterotot, ${ }^{37}$ G. Touquet, ${ }^{37}$ M. Vander Donckt, ${ }^{37}$ S. Viret, ${ }^{37}$ T. Toriashvili, ${ }^{38, p}$ Z. Tsamalaidze, ${ }^{39, k}$ C. Autermann, ${ }^{40}$ L. Feld, ${ }^{40}$ M. K. Kiesel, ${ }^{40}$ K. Klein, ${ }^{40}$ M. Lipinski, ${ }^{40}$ D. Meuser, ${ }^{40}$ A. Pauls, ${ }^{40}$ M. Preuten, ${ }^{40}$ M. P. Rauch, ${ }^{40}$ J. Schulz,${ }^{40}$ M. Teroerde,${ }^{40}$ B. Wittmer, ${ }^{40}$ M. Erdmann, ${ }^{41}$ B. Fischer, ${ }^{41}$ S. Ghosh, ${ }^{41}$ T. Hebbeker, ${ }^{41}$ K. Hoepfner, ${ }^{41}$ H. Keller, ${ }^{41}$ L. Mastrolorenzo, ${ }^{41}$ M. Merschmeyer, ${ }^{41}$ A. Meyer, ${ }^{41}$ P. Millet ${ }^{41}$ G. Mocellin, ${ }^{41}$ S. Mondal,${ }^{41}$ S. Mukherjee, ${ }^{41}$ D. Noll, ${ }^{41}$ A. Novak, ${ }^{41}$ T. Pook, ${ }^{41}$ A. Pozdnyakov, ${ }^{41}$ T. Quast, ${ }^{41}$ M. Radziej, ${ }^{41}$ Y. Rath, ${ }^{41}$ H. Reithler, ${ }^{41}$ J. Roemer, ${ }^{41}$ A. Schmidt, ${ }^{41}$ S. C. Schuler, ${ }^{41}$ A. Sharma, ${ }^{41}$ S. Wiedenbeck,${ }^{41}$ S. Zaleski, ${ }^{41}$ G. Flügge, ${ }^{42}$ W. Haj Ahmad, ${ }^{42, q}$ O. Hlushchenko, ${ }^{42}$ T. Kress ${ }^{42}$ T. Müller, ${ }^{42}$ A. Nowack, ${ }^{42}$ C. Pistone ${ }^{42}$ O. Pooth, ${ }^{42}$ D. Roy, ${ }^{42}$ H. Sert ${ }^{42}$ A. Stahl,${ }^{42, \mathrm{r}}$ M. Aldaya Martin, ${ }^{43}$ P. Asmuss, ${ }^{43}$ I. Babounikau, ${ }^{43}$ H. Bakhshiansohi ${ }^{43}$ K. Beernaert, ${ }^{43}$ O. Behnke, ${ }^{43}$ A. Bermúdez Martínez, ${ }^{43}$ D. Bertsche, ${ }^{43}$ A. A. Bin Anuar, ${ }^{43}$ K. Borras, ${ }^{43, s}$ V. Botta, ${ }^{43}$ A. Campbell, ${ }^{43}$ A. Cardini, ${ }^{43}$ P. Connor, ${ }^{43}$ S. Consuegra Rodríguez, ${ }^{43}$ C. Contreras-Campana, ${ }^{43}$ V. Danilov, ${ }^{43}$ A. De Wit, ${ }^{43}$ M. M. Defranchis, ${ }^{43}$ C. Diez Pardos, ${ }^{43}$ D. Domínguez Damiani, ${ }^{43}$ G. Eckerlin, ${ }^{43}$ D. Eckstein, ${ }^{43}$ T. Eichhorn, ${ }^{43}$ A. Elwood, ${ }^{43}$ E. Eren, ${ }^{43}$ E. Gallo, ${ }^{43, t}$ A. Geiser, ${ }^{43}$ A. Grohsjean, ${ }^{43}$ M. Guthoff, ${ }^{43}$ M. Haranko, ${ }^{43}$ A. Harb, ${ }^{43}$ A. Jafari, ${ }^{43}$ N. Z. Jomhari, ${ }^{43}$ H. Jung, ${ }^{43}$ A. Kasem, ${ }^{43,5}$ M. Kasemann, ${ }^{43}$ H. Kaveh, ${ }^{43}$ J. Keaveney, ${ }^{43}$ C. Kleinwort, ${ }^{43}$ J. Knolle, ${ }^{43}$ D. Krücker, ${ }^{43}$ W. Lange, ${ }^{43}$ T. Lenz, ${ }^{43}$ J. Lidrych, ${ }^{43}$ K. Lipka, ${ }^{43}$ W. Lohmann, ${ }^{43, u}$ R. Mankel, ${ }^{43}$ I.-A. Melzer-Pellmann, ${ }^{43}$ A. B. Meyer, ${ }^{43}$ M. Meyer, ${ }^{43}$ M. Missiroli, ${ }^{43}$ G. Mittag, ${ }^{43}$ J. Mnich, ${ }^{43}$ A. Mussgiller, ${ }^{43}$ V. Myronenko, ${ }^{43}$ D. Pérez Adán, ${ }^{43}$ S. K. Pflitsch, ${ }^{43}$ D. Pitzl, ${ }^{43}$ A. Raspereza, ${ }^{43}$ A. Saibel,${ }^{43}$ M. Savitskyi ${ }^{43}$ V. Scheurer, ${ }^{43}$ P. Schütze,${ }^{43}$ C. Schwanenberger, ${ }^{43}$ R. Shevchenko, ${ }^{43}$ A. Singh ${ }^{43}$ H. Tholen, ${ }^{43}$ O. Turkot, ${ }^{43}$ A. Vagnerini, ${ }^{43}$ M. Van De Klundert, ${ }^{43}$ R. Walsh,${ }^{43}$ Y. Wen, ${ }^{43}$ K. Wichmann, ${ }^{43}$ C. Wissing, ${ }^{43}$ O. Zenaiev, ${ }^{43}$ R. Zlebcik, ${ }^{43}$ R. Aggleton, ${ }^{44}$ S. Bein, ${ }^{44}$ L. Benato, ${ }^{44}$ A. Benecke, ${ }^{44}$ V. Blobel, ${ }^{44}$ T. Dreyer,${ }^{44}$ A. Ebrahimi, ${ }^{44}$ F. Feindt, ${ }^{44}$ A. Fröhlich, ${ }^{44}$ C. Garbers, ${ }^{44}$ E. Garutti, ${ }^{44}$ D. Gonzalez, ${ }^{44}$ P. Gunnellini, ${ }^{44}$ J. Haller, ${ }^{44}$ A. Hinzmann, ${ }^{44}$ A. Karavdina, ${ }^{44}$ G. Kasieczka, ${ }^{44}$ R. Klanner,${ }^{44}$ R. Kogler, ${ }^{44}$ N. Kovalchuk, ${ }^{44}$ S. Kurz, ${ }^{44}$ V. Kutzner, ${ }^{44}$ J. Lange,${ }^{44}$ T. Lange, ${ }^{44}$ A. Malara, ${ }^{44}$ J. Multhaup, ${ }^{44}$ C. E. N. Niemeyer, ${ }^{44}$ A. Perieanu, ${ }^{44}$ A. Reimers,${ }^{44}$ O. Rieger, ${ }^{44}$ C. Scharf ${ }^{44}$ P. Schleper, ${ }^{44}$ S. Schumann, ${ }^{44}$ J. Schwandt, ${ }^{44}$ J. Sonneveld, ${ }^{44}$ H. Stadie, ${ }^{44}$ G. Steinbrück, ${ }^{44}$ F. M. Stober, ${ }^{44}$ B. Vormwald, ${ }^{44}$ I. Zoi, ${ }^{44}$ M. Akbiyik, ${ }^{45}$ C. Barth,${ }^{45}$ M. Baselga, ${ }^{45}$ S. Baur, ${ }^{45}$ T. Berger ${ }^{45}$ E. Butz,${ }^{45}$ R. Caspart, ${ }^{45}$ T. Chwalek, ${ }^{45}$ W. De Boer, ${ }^{45}$ A. Dierlamm, ${ }^{45}$ K. El Morabit, ${ }^{45}$ N. Faltermann, ${ }^{45}$ M. Giffels, ${ }^{45}$ P. Goldenzweig, ${ }^{45}$ A. Gottmann, ${ }^{45}$ M. A. Harrendorf, ${ }^{45}$ F. Hartmann, ${ }^{45, \mathrm{r}}$ U. Husemann, ${ }^{45}$ S. Kudella, ${ }^{45}$ S. Mitra ${ }^{45}$ M. U. Mozer, ${ }^{45}$ D. Müller, ${ }^{45}$ Th. Müller, ${ }^{45}$ M. Musich, ${ }^{45}$ A. Nürnberg, ${ }^{45}$ G. Quast, ${ }^{45}$ K. Rabbertz, ${ }^{45}$ M. Schröder, ${ }^{45}$ I. Shvetsov, ${ }^{45}$ H. J. Simonis, ${ }^{45}$ R. Ulrich, ${ }^{45}$ M. Wassmer, ${ }^{45}$ M. Weber, ${ }^{45}$ C. Wöhrmann, ${ }^{45}$ R. Wolf, ${ }^{45}$ G. Anagnostou, ${ }^{46}$ P. Asenov, ${ }^{46}$ G. Daskalakis, ${ }^{46}$ T. Geralis, ${ }^{46}$ A. Kyriakis, ${ }^{46}$ 
D. Loukas, ${ }^{46}$ G. Paspalaki, ${ }^{46}$ M. Diamantopoulou, ${ }^{47}$ G. Karathanasis, ${ }^{47}$ P. Kontaxakis, ${ }^{47}$ A. Manousakis-katsikakis, ${ }^{47}$ A. Panagiotou, ${ }^{47}$ I. Papavergou, ${ }^{47}$ N. Saoulidou, ${ }^{47}$ A. Stakia, ${ }^{47}$ K. Theofilatos, ${ }^{47}$ K. Vellidis, ${ }^{47}$ E. Vourliotis, ${ }^{47}$ G. Bakas, ${ }^{48}$ K. Kousouris, ${ }^{48}$ I. Papakrivopoulos, ${ }^{48}$ G. Tsipolitis, ${ }^{48}$ I. Evangelou, ${ }^{49}$ C. Foudas, ${ }^{49}$ P. Gianneios, ${ }^{49}$ P. Katsoulis, ${ }^{49}$ P. Kokkas, ${ }^{49}$ S. Mallios, ${ }^{49}$ K. Manitara, ${ }^{49}$ N. Manthos, ${ }^{49}$ I. Papadopoulos, ${ }^{49}$ J. Strologas, ${ }^{49}$ F. A. Triantis, ${ }^{49}$ D. Tsitsonis, ${ }^{49}$ M. Bartók, ${ }^{50, v}$ R. Chudasama, ${ }^{50}$ M. Csanad, ${ }^{50}$ P. Major, ${ }^{50}$ K. Mandal, ${ }^{50}$ A. Mehta, ${ }^{50}$ M. I. Nagy, ${ }^{50}$ G. Pasztor, ${ }^{50}$ O. Surányi, ${ }^{50}$ G. I. Veres, ${ }^{50}$ G. Bencze, ${ }^{51}$ C. Hajdu, ${ }^{51}$ D. Horvath, ${ }^{51, w}$ F. Sikler, ${ }^{51}$ T. Á. Vámi, ${ }^{51}$ V. Veszpremi, ${ }^{51}$ G. Vesztergombi, ${ }^{51, a, x}$ N. Beni, ${ }^{52}$ S. Czellar, ${ }^{52}$ J. Karancsi, ${ }^{52, v}$ A. Makovec, ${ }^{52}$ J. Molnar, ${ }^{52}$ Z. Szillasi, ${ }^{52}$ P. Raics, ${ }^{53}$ D. Teyssier, ${ }^{53}$ Z. L. Trocsanyi, ${ }^{53}$ B. Ujvari, ${ }^{53}$ T. Csorgo, ${ }^{54}$ W. J. Metzger, ${ }^{54}$ F. Nemes, ${ }^{54}$ T. Novak, ${ }^{54}$ S. Choudhury, ${ }^{55}$ J. R. Komaragiri, ${ }^{55}$ P. C. Tiwari, ${ }^{55}$ S. Bahinipati, ${ }^{56, y}$ C. Kar, ${ }^{56}$ G. Kole, ${ }^{56}$ P. Mal, ${ }^{56}$ V. K. Muraleedharan Nair Bindhu, ${ }^{56}$ A. Nayak, ${ }^{56, z}$ D. K. Sahoo, ${ }^{56, y}$ S. K. Swain, ${ }^{56}$ S. Bansal, ${ }^{57}$ S. B. Beri, ${ }^{57}$ V. Bhatnagar, ${ }^{57}$ S. Chauhan, ${ }^{57}$ R. Chawla, ${ }^{57}$ N. Dhingra, ${ }^{57}$ R. Gupta, ${ }^{57}$ A. Kaur, ${ }^{57}$ M. Kaur, ${ }^{57}$ S. Kaur, ${ }^{57}$ P. Kumari, ${ }^{57}$ M. Lohan, ${ }^{57}$ M. Meena, ${ }^{57}$ K. Sandeep, ${ }^{57}$ S. Sharma, ${ }^{57}$ J. B. Singh, ${ }^{57}$ A. K. Virdi, ${ }^{57}$ G. Walia, ${ }^{57}$ A. Bhardwaj, ${ }^{58}$ B. C. Choudhary, ${ }^{58}$ R. B. Garg, ${ }^{58}$ M. Gola, ${ }^{58}$ S. Keshri, ${ }^{58}$ Ashok Kumar, ${ }^{58}$ M. Naimuddin, ${ }^{58}$

P. Priyanka, ${ }^{58}$ K. Ranjan, ${ }^{58}$ Aashaq Shah, ${ }^{58}$ R. Sharma, ${ }^{58}$ R. Bhardwaj, ${ }^{59, \text { aa }}$ M. Bharti, ${ }^{59, \text { aa }}$ R. Bhattacharya, ${ }^{59}$ S. Bhattacharya, ${ }^{59}$ U. Bhawandeep, ${ }^{59, \text { aa }}$ D. Bhowmik, ${ }^{59}$ S. Dutta, ${ }^{59}$ S. Ghosh, ${ }^{59}$ M. Maity, ${ }^{59, b b}$ K. Mondal, ${ }^{59}$ S. Nandan, ${ }^{59}$ A. Purohit, ${ }^{59}$ P. K. Rout, ${ }^{59}$ G. Saha, ${ }^{59}$ S. Sarkar, ${ }^{59}$ T. Sarkar, ${ }^{59, \text { bb }}$ M. Sharan, ${ }^{59}$ B. Singh, ${ }^{59, \text { aa }}$ S. Thakur, ${ }^{59, \text { aa }}$ P. K. Behera, ${ }^{60}$

P. Kalbhor, ${ }^{60}$ A. Muhammad, ${ }^{60}$ P. R. Pujahari, ${ }^{60}$ A. Sharma, ${ }^{60}$ A. K. Sikdar, ${ }^{60}$ D. Dutta, ${ }^{61}$ V. Jha, ${ }^{61}$ V. Kumar, ${ }^{61}$

D. K. Mishra, ${ }^{61}$ P. K. Netrakanti, ${ }^{61}$ L. M. Pant, ${ }^{61}$ P. Shukla, ${ }^{61}$ T. Aziz, ${ }^{62}$ M. A. Bhat, ${ }^{62}$ S. Dugad, ${ }^{62}$ G. B. Mohanty, ${ }^{62}$ N. Sur, ${ }^{62}$

Ravindra Kumar Verma, ${ }^{62}$ S. Banerjee, ${ }^{63}$ S. Bhattacharya, ${ }^{63}$ S. Chatterjee, ${ }^{63}$ P. Das, ${ }^{63}$ M. Guchait, ${ }^{63}$ S. Karmakar, ${ }^{63}$ S. Kumar, ${ }^{63}$ G. Majumder, ${ }^{63}$ K. Mazumdar, ${ }^{63}$ N. Sahoo, ${ }^{63}$ S. Sawant, ${ }^{63}$ S. Dube, ${ }^{64}$ V. Hegde, ${ }^{64}$ B. Kansal, ${ }^{64}$ A. Kapoor, ${ }^{64}$

K. Kothekar, ${ }^{64}$ S. Pandey, ${ }^{64}$ A. Rane, ${ }^{64}$ A. Rastogi, ${ }^{64}$ S. Sharma, ${ }^{64}$ S. Chenarani, ${ }^{65, c c}$ E. Eskandari Tadavani, ${ }^{65}$

S. M. Etesami, ${ }^{65, c c}$ M. Khakzad, ${ }^{65}$ M. Mohammadi Najafabadi, ${ }^{65}$ M. Naseri, ${ }^{65}$ F. Rezaei Hosseinabadi, ${ }^{65}$ M. Felcini, ${ }^{66}$ M. Grunewald, ${ }^{66}$ M. Abbrescia, ${ }^{67 a, 67 b}$ R. Aly, ${ }^{67 a, 67 b, d d}$ C. Calabria, ${ }^{67 a, 67 b}$ A. Colaleo, ${ }^{67 a}$ D. Creanza, ${ }^{67 a, 67 c}$ L. Cristella, ${ }^{67 a, 67 b}$ N. De Filippis, ${ }^{67 a, 67 c}$ M. De Palma, ${ }^{67 a, 67 b}$ A. Di Florio, ${ }^{67 a, 67 b}$ W. Elmetenawee, ${ }^{67 a, 67 b}$ L. Fiore, ${ }^{67 a}$ A. Gelmi, ${ }^{67 a, 67 b}$ G. Iaselli, ${ }^{67 a, 67 c}$ M. Ince, ${ }^{67 a, 67 b}$ S. Lezki, ${ }^{67 a, 67 b}$ G. Maggi, ${ }^{67 a, 67 c}$ M. Maggi, ${ }^{67 a}$ G. Miniello, ${ }^{67 a, 67 b}$ S. My, ${ }^{67 a, 67 b}$ S. Nuzzo, ${ }^{67 a, 67 b}$ A. Pompili, ${ }^{67 a, 67 b}$ G. Pugliese, ${ }^{67 a, 67 c}$ R. Radogna, ${ }^{67 a}$ A. Ranieri, ${ }^{67 a}$ G. Selvaggi, ${ }^{67 a, 67 b}$ L. Silvestris, ${ }^{67 a}$ F. M. Simone, ${ }^{67 a, 67 b}$ R. Venditti, ${ }^{67 a}$ P. Verwilligen, ${ }^{67 a}$ G. Abbiendi, ${ }^{68 a}$ C. Battilana, ${ }^{68 a, 68 b}$ D. Bonacorsi, ${ }^{68 a, 68 b}$ L. Borgonovi, ${ }^{68,68 b}$

S. Braibant-Giacomelli, ${ }^{68 a, 68 b}$ R. Campanini, ${ }^{68 a, 68 b}$ P. Capiluppi, ${ }^{68 a, 68 b}$ A. Castro, ${ }^{68 a, 68 b}$ F. R. Cavallo, ${ }^{68 a}$ C. Ciocca, ${ }^{68 a}$

G. Codispoti, ${ }^{68 a, 68 b}$ M. Cuffiani, ${ }^{68 a, 68 b}$ G. M. Dallavalle, ${ }^{68 a}$ F. Fabbri, ${ }^{68 a}$ A. Fanfani, ${ }^{68 a, 68 b}$ E. Fontanesi, ${ }^{68 a, 68 b}$

P. Giacomelli, ${ }^{68 a}$ C. Grandi, ${ }^{68 a}$ L. Guiducci, ${ }^{68 a, 68 b}$ F. Iemmi, ${ }^{68 a, 68 b}$ S. Lo Meo, ${ }^{68 a, e e}$ S. Marcellini, ${ }^{68 a}$ G. Masetti, ${ }^{68 a}$ F. L. Navarria, ${ }^{68 a, 68 b}$ A. Perrotta, ${ }^{68 a}$ F. Primavera, ${ }^{68 a, 68 b}$ A. M. Rossi, ${ }^{68 a, 68 b}$ T. Rovelli, ${ }^{68 a, 68 b}$ G. P. Siroli, ${ }^{68 a, 68 b}$ N. Tosi, ${ }^{68 a}$ S. Albergo, ${ }^{69 a, 69 b, f f}$ S. Costa, ${ }^{69 a, 69 b}$ A. Di Mattia, ${ }^{69 a}$ R. Potenza, ${ }^{69 a, 69 b}$ A. Tricomi, ${ }^{69 a, 69 b, f f}$ C. Tuve, ${ }^{69 a, 69 b}$ G. Barbagli, ${ }^{70 a}$ A. Cassese, ${ }^{70 a}$ R. Ceccarelli, ${ }^{70 a}$ V. Ciulli, ${ }^{70 a, 70 b}$ C. Civinini, ${ }^{70 a}$ R. D’Alessandro, ${ }^{70 a, 70 b}$ E. Focardi, ${ }^{70 a, 70 b}$ G. Latino, ${ }^{70 a, 70 b}$ P. Lenzi, ${ }^{70 a, 70 b}$ M. Meschini, ${ }^{70 a}$ S. Paoletti, ${ }^{70 a}$ G. Sguazzoni, ${ }^{70 a}$ L. Viliani, ${ }^{70 a}$ L. Benussi, ${ }^{71}$ S. Bianco, ${ }^{71}$ D. Piccolo, ${ }^{71}$ M. Bozzo, ${ }^{72 a, 72 b}$ F. Ferro, ${ }^{72 a}$ R. Mulargia, ${ }^{72 a, 72 b}$ E. Robutti, ${ }^{72 a}$ S. Tosi, ${ }^{72 a, 72 b}$ A. Benaglia, ${ }^{73 a}$ A. Beschi, ${ }^{73 a, 73 b}$ F. Brivio, ${ }^{73 a, 73 b}$ V. Ciriolo, ${ }^{73 a, 73 b, r}$ S. Di Guida, ${ }^{73 a, 73 b, r}$ M. E. Dinardo, ${ }^{73 a, 73 b}$ P. Dini, ${ }^{73 a}$ S. Gennai, ${ }^{73 a}$ A. Ghezzi, ${ }^{73 a, 73 b}$ P. Govoni, ${ }^{73 a, 73 b}$ L. Guzzi, ${ }^{73 a, 73 b}$ M. Malberti, ${ }^{73 a}$ S. Malvezzi, ${ }^{73 a}$ D. Menasce, ${ }^{73 a}$ F. Monti, ${ }^{73 a, 73 b}$ L. Moroni, ${ }^{73 a}$ M. Paganoni, ${ }^{73 a, 73 b}$

D. Pedrini, ${ }^{73 a}$ S. Ragazzi, ${ }^{73 a, 73 b}$ T. Tabarelli de Fatis, ${ }^{73 a, 73 b}$ D. Zuolo, ${ }^{73 a, 73 b}$ S. Buontempo, ${ }^{74 a}$ N. Cavallo, ${ }^{74 a, 74 c}$

A. De Iorio, ${ }^{74 a, 74 b}$ A. Di Crescenzo, ${ }^{74 a, 74 b}$ F. Fabozzi, ${ }^{74 a, 74 c}$ F. Fienga, ${ }^{74 a}$ G. Galati, ${ }^{74 a}$ A. O. M. Iorio, ${ }^{74 a, 74 b}$ L. Lista, $^{74 a, 74 b}$ S. Meola, ${ }^{74 a, 74 d, r}$ P. Paolucci, ${ }^{74 a, r}$ B. Rossi, ${ }^{74 a}$ C. Sciacca, ${ }^{74 a, 74 b}$ E. Voevodina, ${ }^{74 a, 74 b}$ P. Azzi, ${ }^{75 a}$ N. Bacchetta, ${ }^{75 a}$ D. Bisello, ${ }^{75 a, 75 b}$ A. Boletti, ${ }^{75 a, 75 b}$ A. Bragagnolo, ${ }^{75 a, 75 b}$ R. Carlin, ${ }^{75 a, 75 b}$ P. Checchia, ${ }^{75 a}$ P. De Castro Manzano, ${ }^{75 a}$ T. Dorigo, ${ }^{75 a}$ U. Dosselli, ${ }^{75 a}$ F. Gasparini, ${ }^{75 a, 75 b}$ U. Gasparini, ${ }^{75 a, 75 b}$ A. Gozzelino, ${ }^{75 a}$ S. Y. Hoh, ${ }^{75 a, 75 b}$ P. Lujan, ${ }^{75 a}$ M. Margoni, ${ }^{75 a, 75 b}$ A. T. Meneguzzo, ${ }^{75 a, 75 b}$ J. Pazzini, ${ }^{75 a, 75 b}$ M. Presilla, ${ }^{75 a, 75 b}$ P. Ronchese, ${ }^{75 a, 75 b}$ R. Rossin, ${ }^{75 a, 75 b}$ F. Simonetto, ${ }^{75 a, 75 b}$ A. Tiko, ${ }^{75 a}$ M. Tosi, ${ }^{75 a, 75 b}$ M. Zanetti, ${ }^{75 a, 75 b}$ P. Zotto, ${ }^{75 a, 75 b}$ G. Zumerle, ${ }^{75 a, 75 b}$ A. Braghieri, ${ }^{76 a}$ D. Fiorina, ${ }^{76 a, 76 b}$ P. Montagna, ${ }^{76 a, 76 b}$ S. P. Ratti, ${ }^{76 a, 76 b}$ V. Re, ${ }^{76 a}$ M. Ressegotti, ${ }^{76 a, 76 b}$ C. Riccardi, ${ }^{76 a, 76 b}$ P. Salvini, ${ }^{76 a}$ I. Vai, $^{76 a}$ P. Vitulo, ${ }^{76 a, 76 b}$ M. Biasini, ${ }^{77 a, 77 b}$ G. M. Bilei, ${ }^{77 a}$ D. Ciangottini, ${ }^{77 a, 77 b}$ L. Fanò, ${ }^{77 a, 77 b}$ P. Lariccia, ${ }^{77 a, 77 b}$ R. Leonardi, ${ }^{77 a, 77 b}$ E. Manoni, ${ }^{77 a}$ G. Mantovani, ${ }^{77 a, 77 b}$ V. Mariani, ${ }^{77 a, 77 b}$ M. Menichelli, ${ }^{77 a}$ A. Rossi, ${ }^{77 a, 77 b}$ A. Santocchia, ${ }^{77 a, 77 b}$ D. Spiga, ${ }^{77 a}$

K. Androsov, ${ }^{78 a}$ P. Azzurri, ${ }^{78 a}$ G. Bagliesi, ${ }^{78 a}$ V. Bertacchi, ${ }^{78 a, 78 c}$ L. Bianchini, ${ }^{78 a}$ T. Boccali, ${ }^{78 a}$ R. Castaldi, ${ }^{78 a}$

M. A. Ciocci, ${ }^{78 a, 78 b}$ R. Dell'Orso, ${ }^{78 a}$ G. Fedi, ${ }^{78 a}$ L. Giannini, ${ }^{78 a, 78 c}$ A. Giassi, ${ }^{78 a}$ M. T. Grippo, ${ }^{78 a}$ F. Ligabue, ${ }^{78 a, 78 c}$ E. Manca, ${ }^{78 a, 78 c}$ G. Mandorli, ${ }^{78 a, 78 c}$ A. Messineo, ${ }^{78 a, 78 b}$ F. Palla, ${ }^{78 a}$ A. Rizzi, ${ }^{78 a, 78 b}$ G. Rolandi, ${ }^{78 a, g g}$ S. Roy Chowdhury, ${ }^{78 a}$ 
A. Scribano, ${ }^{78 \mathrm{a}}$ P. Spagnolo, ${ }^{78 \mathrm{a}}$ R. Tenchini, ${ }^{78 \mathrm{a}}$ G. Tonelli, ${ }^{78 a, 78 \mathrm{~b}}$ N. Turini ${ }^{78 \mathrm{a}}$ A. Venturi, ${ }^{78 \mathrm{a}}$ P. G. Verdini, ${ }^{78 \mathrm{a}}$ F. Cavallari, ${ }^{79 a}$ M. Cipriani, ${ }^{79 a, 79 b}$ D. Del Re,${ }^{79 a, 79 b}$ E. Di Marco, ${ }^{79 a, 79 b}$ M. Diemoz, ${ }^{79 a}$ E. Longo, ${ }^{79 a, 79 b}$ P. Meridiani, ${ }^{79 a}$ G. Organtini, ${ }^{79 a, 79 b}$ F. Pandolfi, ${ }^{79 a}$ R. Paramatti, ${ }^{79,79 b}$ C. Quaranta, ${ }^{79 a, 79 b}$ S. Rahatlou, ${ }^{79 a, 79 b}$ C. Rovelli, ${ }^{79 a}$ F. Santanastasio, ${ }^{79 a, 79 b}$ L. Soffi, ${ }^{79 a, 79 b}$ N. Amapane, ${ }^{80 a, 80 b}$ R. Arcidiacono, ${ }^{80 a, 80 c}$ S. Argiro, ${ }^{80 a, 80 b}$ M. Arneodo, ${ }^{80 a, 80 \mathrm{c}}$ N. Bartosik, ${ }^{80 \mathrm{a}}$ R. Bellan, ${ }^{80 \mathrm{a}, 80 \mathrm{~b}}$ A. Bellora, ${ }^{80 \mathrm{a}}$ C. Biino, ${ }^{80 a}$ A. Cappati, ${ }^{80 a, 80 b}$ N. Cartiglia, ${ }^{80 a}$ S. Cometti, ${ }^{80 a}$ M. Costa, ${ }^{80 a, 80 b}$ R. Covarelli, ${ }^{80 a, 80 b}$ N. Demaria, ${ }^{80 a}$

B. Kiani ${ }^{80 \mathrm{a}, 80 \mathrm{~b}}$ F. Legger, ${ }^{80 \mathrm{a}}$ C. Mariotti ${ }^{80 \mathrm{a}}$ S. Maselli, ${ }^{80 \mathrm{a}}$ E. Migliore, ${ }^{80 \mathrm{a}, 80 \mathrm{~b}}$ V. Monaco, ${ }^{80 \mathrm{a}, 80 \mathrm{~b}}$ E. Monteil, ${ }^{80 \mathrm{a}, 80 \mathrm{~b}}$ M. Monteno, ${ }^{80 \mathrm{a}}$ M. M. Obertino,${ }^{80 a, 80 \mathrm{~b}}$ G. Ortona, ${ }^{80 \mathrm{a}, 80 \mathrm{~b}}$ L. Pacher ${ }^{80 \mathrm{a}, 80 \mathrm{~b}}$ N. Pastrone, ${ }^{80 \mathrm{a}}$ M. Pelliccioni,${ }^{80 \mathrm{a}}$ G. L. Pinna Angioni, ${ }^{80 a, 80 b}$ A. Romero, ${ }^{80 a, 80 b}$ M. Ruspa,${ }^{80 a, 80 \mathrm{c}}$ R. Salvatico, ${ }^{80 a, 80 b}$ V. Sola, ${ }^{80 \mathrm{a}}$ A. Solano, ${ }^{80 \mathrm{a}, 80 \mathrm{~b}}$ D. Soldi, ${ }^{80 \mathrm{a}, 80 \mathrm{~b}}$ A. Staiano, ${ }^{80 a}$ S. Belforte, ${ }^{81 a}$ V. Candelise,${ }^{81 a, 81 b}$ M. Casarsa, ${ }^{81 a}$ F. Cossutti, ${ }^{81 a}$ A. Da Rold, ${ }^{81 a, 81 b}$ G. Della Ricca, ${ }^{81 a, 81 b}$ F. Vazzoler, ${ }^{81 a, 81 b}$ A. Zanetti, ${ }^{81 a}$ B. Kim, ${ }^{82}$ D. H. Kim, ${ }^{82}$ G. N. Kim, ${ }^{82}$ J. Lee, ${ }^{82}$ S. W. Lee, ${ }^{82}$ C. S. Moon, ${ }^{82}$ Y. D. Oh, ${ }^{82}$ S. I. Pak, ${ }^{82}$ S. Sekmen, ${ }^{82}$ D. C. Son, ${ }^{82}$ Y. C. Yang, ${ }^{82}$ H. Kim,${ }^{83}$ D. H. Moon, ${ }^{83}$ G. Oh, ${ }^{83}$ B. Francois, ${ }^{84}$ T. J. Kim, ${ }^{84}$ J. Park, ${ }^{84}$ S. Cho, ${ }^{85}$ S. Choi, ${ }^{85}$ Y. Go, ${ }^{85}$ S. Ha ${ }^{85}$ B. Hong, ${ }^{85}$ K. Lee, ${ }^{85}$ K. S. Lee ${ }^{85}$ J. Lim,${ }^{85}$ J. Park ${ }^{85}$ S. K. Park, ${ }^{85}$ Y. Roh,${ }^{85}$ J. Yoo, ${ }^{85}$ J. Goh, ${ }^{86}$ H. S. Kim,${ }^{87}$ J. Almond, ${ }^{88}$ J. H. Bhyun, ${ }^{88}$ J. Choi, ${ }^{88}$ S. Jeon, ${ }^{88}$ J. Kim,${ }^{88}$ J. S. Kim, ${ }^{88}$ H. Lee, ${ }^{88}$ K. Lee, ${ }^{88}$ S. Lee,${ }^{88}$ K. Nam ${ }^{88}$ M. Oh, ${ }^{88}$ S. B. Oh, ${ }^{88}$ B. C. Radburn-Smith, ${ }^{88}$ U. K. Yang, ${ }^{88}$ H. D. Yoo, ${ }^{88}$ I. Yoon, ${ }^{88}$ G. B. Yu, ${ }^{88}$ D. Jeon, ${ }^{89}$ H. Kim, ${ }^{89}$ J. H. Kim, ${ }^{89}$ J. S. H. Lee, ${ }^{89}$ I. C. Park,${ }^{89}$ I. J. Watson, ${ }^{89}$ Y. Choi,${ }^{90}$ C. Hwang, ${ }^{90}$ Y. Jeong, ${ }^{90}$ J. Lee, ${ }^{90}$ Y. Lee, ${ }^{90}$ I. Yu, ${ }^{90}$ V. Veckalns,${ }^{91, \text { hh }}$ V. Dudenas, ${ }^{92}$ A. Juodagalvis, ${ }^{92}$ A. Rinkevicius, ${ }^{92}$ G. Tamulaitis, ${ }^{92}$ J. Vaitkus, ${ }^{92}$ Z. A. Ibrahim, ${ }^{93}$ F. Mohamad Idris, ${ }^{93, \text { ii }}$ W. A. T. Wan Abdullah, ${ }^{93}$ M. N. Yusli, ${ }^{93}$ Z. Zolkapli,${ }^{93}$ J. F. Benitez, ${ }^{94}$ A. Castaneda Hernandez, ${ }^{94}$ J. A. Murillo Quijada, ${ }^{94}$ L. Valencia Palomo, ${ }^{94}$ H. Castilla-Valdez, ${ }^{95}$ E. De La Cruz-Burelo, ${ }^{95}$ I. Heredia-De La Cruz, ${ }^{95, j j}$ R. Lopez-Fernandez ${ }^{95}$ A. Sanchez-Hernandez, ${ }^{95}$ S. Carrillo Moreno, ${ }^{96}$ C. Oropeza Barrera, ${ }^{96}$ M. Ramirez-Garcia, ${ }^{96}$ F. Vazquez Valencia, ${ }^{96}$ J. Eysermans,${ }^{97}$ I. Pedraza,${ }^{97}$ H. A. Salazar Ibarguen,${ }^{97}$ C. Uribe Estrada, ${ }^{97}$ A. Morelos Pineda, ${ }^{98}$ J. Mijuskovic, ${ }^{99}$ N. Raicevic, ${ }^{99}$ D. Krofcheck, ${ }^{100}$ S. Bheesette, ${ }^{101}$ P. H. Butler, ${ }^{101}$ A. Ahmad, ${ }^{102}$ M. Ahmad, ${ }^{102}$ Q. Hassan, ${ }^{102}$ H. R. Hoorani, ${ }^{102}$ W. A. Khan, ${ }^{102}$ M. A. Shah, ${ }^{102}$ M. Shoaib, ${ }^{102}$ M. Waqas, ${ }^{102}$ V. Avati, ${ }^{103}$ L. Grzanka, ${ }^{103}$ M. Malawski, ${ }^{103}$ H. Bialkowska, ${ }^{104}$ M. Bluj, ${ }^{104}$ B. Boimska, ${ }^{104}$ M. Górski, ${ }^{104}$ M. Kazana, ${ }^{104}$ M. Szleper, ${ }^{104}$ P. Zalewski, ${ }^{104}$ K. Bunkowski, ${ }^{105}$ A. Byszuk, ${ }^{105, \mathrm{kk}}$ K. Doroba, ${ }^{105}$ A. Kalinowski, ${ }^{105}$ M. Konecki, ${ }^{105}$ J. Krolikowski, ${ }^{105}$ M. Misiura, ${ }^{105}$ M. Olszewski, ${ }^{105}$ M. Walczak, ${ }^{105}$ M. Araujo, ${ }^{106}$ P. Bargassa, ${ }^{106}$ D. Bastos, ${ }^{106}$ A. Di Francesco, ${ }^{106}$ P. Faccioli, ${ }^{106}$ B. Galinhas, ${ }^{106}$ M. Gallinaro, ${ }^{106}$ J. Hollar, ${ }^{106}$ N. Leonardo, ${ }^{106}$ T. Niknejad, ${ }^{106}$ J. Seixas, ${ }^{106}$ K. Shchelina, ${ }^{106}$ G. Strong, ${ }^{106}$ O. Toldaiev, ${ }^{106}$ J. Varela ${ }^{106}$ S. Afanasiev, ${ }^{107}$ P. Bunin,${ }^{107}$ M. Gavrilenko, ${ }^{107}$ I. Golutvin, ${ }^{107}$ I. Gorbunov, ${ }^{107}$ A. Kamenev, ${ }^{107}$ V. Karjavine, ${ }^{107}$ A. Lanev ${ }^{107}$ A. Malakhov, ${ }^{107}$ V. Matveev,${ }^{107,11, m m}$ P. Moisenz, ${ }^{107}$ V. Palichik, ${ }^{107}$ V. Perelygin, ${ }^{107}$ M. Savina,${ }^{107}$ S. Shmatov ${ }^{107}$ S. Shulha, ${ }^{107}$ N. Skatchkov, ${ }^{107}$ V. Smirnov, ${ }^{107}$ N. Voytishin, ${ }^{107}$ A. Zarubin, ${ }^{107}$ L. Chtchipounov, ${ }^{108}$ V. Golovtcov, ${ }^{108}$ Y. Ivanov, ${ }^{108}$ V. Kim, ${ }^{108, \text { nn }}$ E. Kuznetsova, ${ }^{108,00}$ P. Levchenko, ${ }^{108}$ V. Murzin, ${ }^{108}$ V. Oreshkin, ${ }^{108}$ I. Smirnov, ${ }^{108}$ D. Sosnov, ${ }^{108}$ V. Sulimov, ${ }^{108}$ L. Uvarov, ${ }^{108}$ A. Vorobyev, ${ }^{108}$ Yu. Andreev, ${ }^{109}$ A. Dermenev, ${ }^{109}$ S. Gninenko, ${ }^{109}$ N. Golubev, ${ }^{109}$ A. Karneyeu, ${ }^{109}$ M. Kirsanov, ${ }^{109}$ N. Krasnikov, ${ }^{109}$ A. Pashenkov, ${ }^{109}$ D. Tlisov, ${ }^{109}$ A. Toropin, ${ }^{109}$ V. Epshteyn, ${ }^{110}$ V. Gavrilov, ${ }^{110}$ N. Lychkovskaya, ${ }^{110}$ A. Nikitenko, ${ }^{110, p p}$ V. Popov, ${ }^{110}$ I. Pozdnyakov, ${ }^{110}$ G. Safronov, ${ }^{110}$ A. Spiridonov, ${ }^{110}$ A. Stepennov, ${ }^{110}$ M. Toms, ${ }^{110}$ E. Vlasov, ${ }^{110}$ A. Zhokin, ${ }^{110}$ T. Aushev, ${ }^{111}$ O. Bychkova, ${ }^{112}$ R. Chistov, ${ }^{112, q q}$ M. Danilov, ${ }^{112, q q}$ S. Polikarpov, ${ }^{112, q q}$ E. Tarkovskii, ${ }^{112}$ V. Andreev, ${ }^{113}$ M. Azarkin, ${ }^{113}$ I. Dremin, ${ }^{113}$ M. Kirakosyan, ${ }^{113}$ A. Terkulov, ${ }^{113}$ A. Baskakov, ${ }^{114}$ A. Belyaev, ${ }^{114}$ E. Boos, ${ }^{114}$ V. Bunichev, ${ }^{114}$ M. Dubinin, ${ }^{114, r r}$ L. Dudko, ${ }^{114}$ A. Ershov, ${ }^{114}$ V. Klyukhin, ${ }^{114}$ O. Kodolova, ${ }^{114}$ I. Lokhtin, ${ }^{114}$ S. Obraztsov, ${ }^{114}$ S. Petrushanko, ${ }^{114}$ V. Savrin, ${ }^{114}$ A. Barnyakov, ${ }^{115, \text { ss }}$ V. Blinov, ${ }^{115, s s}$ T. Dimova, ${ }^{115, \text { ss }}$ L. Kardapoltsev, ${ }^{115, \text { ss }}$ Y. Skovpen, ${ }^{115, \text { ss }}$ I. Azhgirey, ${ }^{116}$ I. Bayshev, ${ }^{116}$ S. Bitioukov, ${ }^{116}$ V. Kachanov, ${ }^{116}$ D. Konstantinov, ${ }^{116}$ P. Mandrik, ${ }^{116}$ V. Petrov ${ }^{116}$ R. Ryutin, ${ }^{116}$ S. Slabospitskii, ${ }^{116}$ A. Sobol, ${ }^{116}$ S. Troshin, ${ }^{116}$ N. Tyurin, ${ }^{116}$ A. Uzunian, ${ }^{116}$ A. Volkov, ${ }^{116}$ A. Babaev, ${ }^{117}$ A. Iuzhakov, ${ }^{117}$ V. Okhotnikov, ${ }^{117}$ V. Borchsh, ${ }^{118}$ V. Ivanchenko, ${ }^{118}$ E. Tcherniaev, ${ }^{118}$ P. Adzic, ${ }^{119, \text { tt }}$ P. Cirkovic, ${ }^{119}$ M. Dordevic, ${ }^{119}$ P. Milenovic, ${ }^{119}$ J. Milosevic, ${ }^{119}$ M. Stojanovic, ${ }^{119}$ M. Aguilar-Benitez, ${ }^{120}$ J. Alcaraz Maestre, ${ }^{120}$ A. Álvarez Fernández, ${ }^{120}$ I. Bachiller, ${ }^{120}$ M. Barrio Luna, ${ }^{120}$ J. A. Brochero Cifuentes, ${ }^{120}$ C. A. Carrillo Montoya, ${ }^{120}$ M. Cepeda, ${ }^{120}$ M. Cerrada, ${ }^{120}$ N. Colino, ${ }^{120}$ B. De La Cruz, ${ }^{120}$ A. Delgado Peris, ${ }^{120}$ C. Fernandez Bedoya, ${ }^{120}$ J. P. Fernández Ramos, ${ }^{120}$ J. Flix, ${ }^{120}$ M. C. Fouz, ${ }^{120}$ O. Gonzalez Lopez, ${ }^{120}$ S. Goy Lopez, ${ }^{120}$ J. M. Hernandez, ${ }^{120}$ M. I. Josa, ${ }^{120}$ D. Moran, ${ }^{120}$ Á. Navarro Tobar, ${ }^{120}$ A. Pérez-Calero Yzquierdo, ${ }^{120}$ J. Puerta Pelayo, ${ }^{120}$ I. Redondo, ${ }^{120}$ L. Romero, ${ }^{120}$ S. Sánchez Navas, ${ }^{120}$ M. S. Soares, ${ }^{120}$ A. Triossi, ${ }^{120}$ C. Willmott, ${ }^{120}$ C. Albajar, ${ }^{121}$ J. F. de Trocóniz, ${ }^{121}$ R. Reyes-Almanza, ${ }^{121}$ B. Alvarez Gonzalez, ${ }^{122}$ J. Cuevas, ${ }^{122}$ C. Erice, ${ }^{122}$ J. Fernandez Menendez, ${ }^{122}$ S. Folgueras, ${ }^{122}$ I. Gonzalez Caballero, ${ }^{122}$ J. R. González Fernández, ${ }^{122}$ E. Palencia Cortezon, ${ }^{122}$ V. Rodríguez Bouza, ${ }^{122}$ S. Sanchez Cruz, ${ }^{122}$ I. J. Cabrillo, ${ }^{123}$ A. Calderon, ${ }^{123}$ B. Chazin Quero, ${ }^{123}$ J. Duarte Campderros, ${ }^{123}$ 
M. Fernandez, ${ }^{123}$ P. J. Fernández Manteca, ${ }^{123}$ A. García Alonso, ${ }^{123}$ G. Gomez, ${ }^{123}$ C. Martinez Rivero, ${ }^{123}$ P. Martinez Ruiz del Arbol, ${ }^{123}$ F. Matorras, ${ }^{123}$ J. Piedra Gomez, ${ }^{123}$ C. Prieels, ${ }^{123}$ T. Rodrigo, ${ }^{123}$ A. Ruiz-Jimeno, ${ }^{123}$ L. Russo, ${ }^{123, \text { uu }}$ L. Scodellaro, ${ }^{123}$ I. Vila, ${ }^{123}$ J. M. Vizan Garcia, ${ }^{123}$ K. Malagalage, ${ }^{124}$ W. G. D. Dharmaratna, ${ }^{125}$ N. Wickramage, ${ }^{125}$ D. Abbaneo, ${ }^{126}$ B. Akgun, ${ }^{126}$ E. Auffray, ${ }^{126}$ G. Auzinger, ${ }^{126}$ J. Baechler, ${ }^{126}$ P. Baillon, ${ }^{126}$ A. H. Ball, ${ }^{126}$ D. Barney, ${ }^{126}$ J. Bendavid, ${ }^{126}$ M. Bianco, ${ }^{126}$ A. Bocci, ${ }^{126}$ P. Bortignon, ${ }^{126}$ E. Bossini, ${ }^{126}$ C. Botta, ${ }^{126}$ E. Brondolin, ${ }^{126}$ T. Camporesi, ${ }^{126}$ A. Caratelli, ${ }^{126}$ G. Cerminara, ${ }^{126}$ E. Chapon, ${ }^{126}$ G. Cucciati, ${ }^{126}$ D. d'Enterria, ${ }^{126}$ A. Dabrowski, ${ }^{126}$ N. Daci, ${ }^{126}$ V. Daponte, ${ }^{126}$ A. David, ${ }^{126}$ O. Davignon, ${ }^{126}$ A. De Roeck, ${ }^{126}$ M. Deile,${ }^{126}$ M. Dobson, ${ }^{126}$ M. Dünser, ${ }^{126}$ N. Dupont, ${ }^{126}$ A. Elliott-Peisert, ${ }^{126}$ N. Emriskova, ${ }^{126}$ F. Fallavollita, ${ }^{126, v v}$ D. Fasanella, ${ }^{126}$ S. Fiorendi, ${ }^{126}$ G. Franzoni, ${ }^{126}$ J. Fulcher, ${ }^{126}$ W. Funk, ${ }^{126}$ S. Giani, ${ }^{126}$ D. Gigi, ${ }^{126}$ A. Gilbert, ${ }^{126}$ K. Gill, ${ }^{126}$ F. Glege, ${ }^{126}$ L. Gouskos, ${ }^{126}$ M. Gruchala, ${ }^{126}$ M. Guilbaud, ${ }^{126}$ D. Gulhan, ${ }^{126}$ J. Hegeman, ${ }^{126}$ C. Heidegger, ${ }^{126}$ Y. Iiyama, ${ }^{126}$ V. Innocente, ${ }^{126}$ T. James, ${ }^{126}$ P. Janot, ${ }^{126}$ O. Karacheban, ${ }^{126, u}$ J. Kaspar, ${ }^{126}$ J. Kieseler, ${ }^{126}$ M. Krammer, ${ }^{126, b}$ N. Kratochwil, ${ }^{126}$ C. Lange, ${ }^{126}$ P. Lecoq, ${ }^{126}$ C. Lourenço, ${ }^{126}$ L. Malgeri, ${ }^{126}$ M. Mannelli, ${ }^{126}$ A. Massironi, ${ }^{126}$ F. Meijers, ${ }^{126}$ J. A. Merlin, ${ }^{126}$ S. Mersi, ${ }^{126}$ E. Meschi, ${ }^{126}$ F. Moortgat, ${ }^{126}$ M. Mulders, ${ }^{126}$ J. Ngadiuba, ${ }^{126}$ J. Niedziela, ${ }^{126}$ S. Nourbakhsh, ${ }^{126}$ S. Orfanelli, ${ }^{126}$ L. Orsini, ${ }^{126}$ F. Pantaleo, ${ }^{126, \mathrm{r}}$ L. Pape, ${ }^{126}$ E. Perez, ${ }^{126}$ M. Peruzzi, ${ }^{126}$ A. Petrilli, ${ }^{126}$ G. Petrucciani, ${ }^{126}$ A. Pfeiffer, ${ }^{126}$ M. Pierini, ${ }^{126}$ F. M. Pitters, ${ }^{126}$ D. Rabady, ${ }^{126}$ A. Racz, ${ }^{126}$ M. Rieger, ${ }^{126}$ M. Rovere, ${ }^{126}$ H. Sakulin, ${ }^{126}$ C. Schäfer, ${ }^{126}$ C. Schwick, ${ }^{126}$ M. Selvaggi, ${ }^{126}$ A. Sharma, ${ }^{126}$ P. Silva, ${ }^{126}$ W. Snoeys, ${ }^{126}$ P. Sphicas, ${ }^{126, w w}$ J. Steggemann, ${ }^{126}$ S. Summers, ${ }^{126}$ V. R. Tavolaro, ${ }^{126}$ D. Treille, ${ }^{126}$ A. Tsirou, ${ }^{126}$ G. P. Van Onsem, ${ }^{126}$ A. Vartak, ${ }^{126}$ M. Verzetti, ${ }^{126}$ W. D. Zeuner, ${ }^{126}$ L. Caminada, ${ }^{127, x x}$ K. Deiters, ${ }^{127}$ W. Erdmann, ${ }^{127}$ R. Horisberger, ${ }^{127}$ Q. Ingram, ${ }^{127}$ H. C. Kaestli, ${ }^{127}$ D. Kotlinski, ${ }^{127}$ U. Langenegger, ${ }^{127}$ T. Rohe, ${ }^{127}$ S. A. Wiederkehr, ${ }^{127}$ M. Backhaus, ${ }^{128}$ P. Berger, ${ }^{128}$ N. Chernyavskaya, ${ }^{128}$ G. Dissertori, ${ }^{128}$ M. Dittmar, ${ }^{128}$ M. Donegà, ${ }^{128}$ C. Dorfer, ${ }^{128}$ T. A. Gómez Espinosa, ${ }^{128}$ C. Grab, ${ }^{128}$ D. Hits, ${ }^{128}$ T. Klijnsma, ${ }^{128}$ W. Lustermann, ${ }^{128}$ R. A. Manzoni, ${ }^{128}$ M. T. Meinhard, ${ }^{128}$ F. Micheli, ${ }^{128}$ P. Musella, ${ }^{128}$ F. Nessi-Tedaldi, ${ }^{128}$ F. Pauss, ${ }^{128}$ G. Perrin, ${ }^{128}$ L. Perrozzi, ${ }^{128}$ S. Pigazzini, ${ }^{128}$ M. G. Ratti, ${ }^{128}$ M. Reichmann, ${ }^{128}$ C. Reissel, ${ }^{128}$ T. Reitenspiess, ${ }^{128}$ D. Ruini, ${ }^{128}$ D. A. Sanz Becerra, ${ }^{128}$ M. Schönenberger, ${ }^{128}$ L. Shchutska, ${ }^{128}$ M. L. Vesterbacka Olsson, ${ }^{128}$ R. Wallny, ${ }^{128}$ D. H. Zhu, ${ }^{128}$ T. K. Aarrestad, ${ }^{129}$ C. Amsler, ${ }^{129, \text { yy }}$ D. Brzhechko, ${ }^{129}$ M. F. Canelli, ${ }^{129}$ A. De Cosa, ${ }^{129}$ R. Del Burgo, ${ }^{129}$ S. Donato, ${ }^{129}$ B. Kilminster, ${ }^{129}$ S. Leontsinis, ${ }^{129}$ V. M. Mikuni, ${ }^{129}$ I. Neutelings, ${ }^{129}$ G. Rauco, ${ }^{129}$ P. Robmann, ${ }^{129}$ K. Schweiger, ${ }^{129}$ C. Seitz, ${ }^{129}$ Y. Takahashi, ${ }^{129}$ S. Wertz, ${ }^{129}$ A. Zucchetta, ${ }^{129}$ T. H. Doan, ${ }^{130}$ C. M. Kuo, ${ }^{130}$ W. Lin, ${ }^{130}$ A. Roy, ${ }^{130}$ S. S. Yu, ${ }^{130}$ P. Chang, ${ }^{131}$ Y. Chao, ${ }^{131}$ K. F. Chen, ${ }^{131}$ P. H. Chen, ${ }^{131}$ W.-S. Hou, ${ }^{131}$ Y. y. Li, ${ }^{131}$ R.-S. Lu, ${ }^{131}$ E. Paganis, ${ }^{131}$ A. Psallidas, ${ }^{131}$ A. Steen, ${ }^{131}$ B. Asavapibhop, ${ }^{132}$ C. Asawatangtrakuldee, ${ }^{132}$ N. Srimanobhas, ${ }^{132}$ N. Suwonjandee, ${ }^{132}$ A. Bat, ${ }^{133}$ F. Boran, ${ }^{133}$ A. Celik, ${ }^{133, z z}$ S. Cerci, ${ }^{133, \text { aaa }}$ S. Damarseckin, ${ }^{133, \text { bbb }}$ Z. S. Demiroglu, ${ }^{133}$ F. Dolek, ${ }^{133}$ C. Dozen, ${ }^{133, c c c}$ I. Dumanoglu, ${ }^{133}$ G. Gokbulut, ${ }^{133}$ Emine Gurpinar Guler, ${ }^{133, \text { ddd }}$ Y. Guler, ${ }^{133}$ I. Hos, ${ }^{133 \text {,eee }}$ C. Isik, ${ }^{133}$ E. E. Kangal, ${ }^{133, f f f}$ O. Kara, ${ }^{133}$

A. Kayis Topaksu, ${ }^{133}$ U. Kiminsu, ${ }^{133}$ G. Onengut, ${ }^{133}$ K. Ozdemir, ${ }^{133, g g g}$ S. Ozturk, ${ }^{133, \text { hhh }}$ A. E. Simsek, ${ }^{133}$

D. Sunar Cerci, ${ }^{133, \text { aaa }}$ U. G. Tok, ${ }^{133}$ S. Turkcapar, ${ }^{133}$ I. S. Zorbakir, ${ }^{133}$ C. Zorbilmez, ${ }^{133}$ B. Isildak, ${ }^{134, \text { iii }}$ G. Karapinar, ${ }^{134, j j j}$ M. Yalvac, ${ }^{134}$ I. O. Atakisi, ${ }^{135}$ E. Gülmez, ${ }^{135}$ M. Kaya, ${ }^{135, k k k}$ O. Kaya, ${ }^{135,111}$ Ö. Özçelik, ${ }^{135}$ S. Tekten, ${ }^{135}$ E. A. Yetkin, ${ }^{135, m m m}$ A. Cakir, ${ }^{136}$ K. Cankocak, ${ }^{136}$ Y. Komurcu, ${ }^{136}$ S. Sen, ${ }^{136, n n n}$ B. Kaynak, ${ }^{137}$ S. Ozkorucuklu, ${ }^{137}$ B. Grynyov, ${ }^{138}$ L. Levchuk, ${ }^{139}$ E. Bhal, ${ }^{140}$ S. Bologna, ${ }^{140}$ J. J. Brooke, ${ }^{140}$ D. Burns, ${ }^{140,000}$ E. Clement, ${ }^{140}$ D. Cussans, ${ }^{140}$ H. Flacher, ${ }^{140}$ J. Goldstein, ${ }^{140}$

G. P. Heath, ${ }^{140}$ H. F. Heath, ${ }^{140}$ L. Kreczko, ${ }^{140}$ B. Krikler, ${ }^{140}$ S. Paramesvaran, ${ }^{140}$ B. Penning, ${ }^{140}$ T. Sakuma, ${ }^{140}$

S. Seif El Nasr-Storey, ${ }^{140}$ V. J. Smith, ${ }^{140}$ J. Taylor, ${ }^{140}$ A. Titterton, ${ }^{140}$ K. W. Bell, ${ }^{141}$ A. Belyaev, ${ }^{141, p p p}$ C. Brew, ${ }^{141}$ R. M. Brown, ${ }^{141}$ D. J. A. Cockerill, ${ }^{141}$ J. A. Coughlan, ${ }^{141}$ K. Harder, ${ }^{141}$ S. Harper, ${ }^{141}$ J. Linacre, ${ }^{141}$ K. Manolopoulos, ${ }^{141}$ D. M. Newbold, ${ }^{141}$ E. Olaiya, ${ }^{141}$ D. Petyt, ${ }^{141}$ T. Reis, ${ }^{141}$ T. Schuh, ${ }^{141}$ C. H. Shepherd-Themistocleous, ${ }^{141}$ A. Thea, ${ }^{141}$ I. R. Tomalin, ${ }^{141}$ T. Williams, ${ }^{141}$ W. J. Womersley, ${ }^{141}$ R. Bainbridge, ${ }^{142}$ P. Bloch, ${ }^{142}$ J. Borg, ${ }^{142}$ S. Breeze, ${ }^{142}$ O. Buchmuller, ${ }^{142}$ A. Bundock, ${ }^{142}$ Gurpreet Singh CHAHAL, ${ }^{142, q q q}$ D. Colling, ${ }^{142}$ P. Dauncey, ${ }^{142}$ G. Davies, ${ }^{142}$ M. Della Negra, ${ }^{142}$ R. Di Maria, ${ }^{142}$ P. Everaerts, ${ }^{142}$ G. Hall, ${ }^{142}$ G. Iles, ${ }^{142}$ M. Komm, ${ }^{142}$ C. Laner, ${ }^{142}$ L. Lyons, ${ }^{142}$ A.-M. Magnan, ${ }^{142}$ S. Malik, ${ }^{142}$ A. Martelli, ${ }^{142}$ V. Milosevic, ${ }^{142}$ A. Morton, ${ }^{142}$ J. Nash, ${ }^{142, r r}$ V. Palladino, ${ }^{142}$ M. Pesaresi, ${ }^{142}$ D. M. Raymond, ${ }^{142}$ A. Richards, ${ }^{142}$ A. Rose, ${ }^{142}$ E. Scott, ${ }^{142}$ C. Seez, ${ }^{142}$ A. Shtipliyski, ${ }^{142}$ M. Stoye, ${ }^{142}$ T. Strebler, ${ }^{142}$ A. Tapper, ${ }^{142}$ K. Uchida, ${ }^{142}$ T. Virdee, ${ }^{142, r}$ N. Wardle, ${ }^{142}$ D. Winterbottom, ${ }^{142}$ J. Wright, ${ }^{142}$ A. G. Zecchinelli, ${ }^{142}$ S. C. Zenz, ${ }^{142}$ J. E. Cole, ${ }^{143}$ P. R. Hobson, ${ }^{143}$ A. Khan, ${ }^{143}$ P. Kyberd, ${ }^{143}$ C. K. Mackay, ${ }^{143}$ I. D. Reid, ${ }^{143}$ L. Teodorescu, ${ }^{143}$ S. Zahid, ${ }^{143}$ K. Call, ${ }^{144}$ B. Caraway, ${ }^{144}$ J. Dittmann, ${ }^{144}$ K. Hatakeyama, ${ }^{144}$ C. Madrid, ${ }^{144}$ B. McMaster, ${ }^{144}$ N. Pastika, ${ }^{144}$ C. Smith, ${ }^{144}$ R. Bartek, ${ }^{145}$ A. Dominguez, ${ }^{145}$ R. Uniyal, ${ }^{145}$ A. M. Vargas Hernandez, ${ }^{145}$ A. Buccilli, ${ }^{146}$ S. I. Cooper, ${ }^{146}$ C. Henderson, ${ }^{146}$ P. Rumerio, ${ }^{146}$ C. West, ${ }^{146}$ A. Albert, ${ }^{147}$ D. Arcaro, ${ }^{147}$ Z. Demiragli, ${ }^{147}$ D. Gastler, ${ }^{147}$ C. Richardson, ${ }^{147}$ 
J. Rohlf, ${ }^{147}$ D. Sperka, ${ }^{147}$ I. Suarez, ${ }^{147}$ L. Sulak, ${ }^{147}$ D. Zou, ${ }^{147}$ G. Benelli, ${ }^{148}$ B. Burkle, ${ }^{148}$ X. Coubez, ${ }^{148, s}$ D. Cutts, ${ }^{148}$ Y. t. Duh, ${ }^{148}$ M. Hadley, ${ }^{148}$ U. Heintz, ${ }^{148}$ J. M. Hogan, ${ }^{148, \text { sss }}$ K. H. M. Kwok, ${ }^{148}$ E. Laird, ${ }^{148}$ G. Landsberg, ${ }^{148}$ K. T. Lau, ${ }^{148}$ J. Lee, ${ }^{148}$ Z. Mao, ${ }^{148}$ M. Narain, ${ }^{148}$ S. Sagir, ${ }^{148, \text { ttt }}$ R. Syarif, ${ }^{148}$ E. Usai, ${ }^{148}$ D. Yu, ${ }^{148}$ W. Zhang, ${ }^{148}$ R. Band, ${ }^{149}$ C. Brainerd, ${ }^{149}$ R. Breedon, ${ }^{149}$ M. Calderon De La Barca Sanchez, ${ }^{149}$ M. Chertok, ${ }^{149}$ J. Conway, ${ }^{149}$ R. Conway, ${ }^{149}$ P. T. Cox, ${ }^{149}$ R. Erbacher, ${ }^{149}$ C. Flores, ${ }^{149}$ G. Funk, ${ }^{149}$ F. Jensen, ${ }^{149}$ W. Ko, ${ }^{149}$ O. Kukral, ${ }^{149}$ R. Lander, ${ }^{149}$ M. Mulhearn, ${ }^{149}$ D. Pellett, ${ }^{149}$ J. Pilot, ${ }^{149}$ M. Shi, ${ }^{149}$ D. Taylor, ${ }^{149}$ K. Tos, ${ }^{149}$ M. Tripathi, ${ }^{149}$ Z. Wang, ${ }^{149}$ F. Zhang, ${ }^{149}$ M. Bachtis, ${ }^{150}$ C. Bravo, ${ }^{150}$ R. Cousins, ${ }^{150}$ A. Dasgupta, ${ }^{150}$ A. Florent, ${ }^{150}$ J. Hauser, ${ }^{150}$ M. Ignatenko, ${ }^{150}$ N. Mccoll, ${ }^{150}$ W. A. Nash, ${ }^{150}$ S. Regnard, ${ }^{150}$ D. Saltzberg, ${ }^{150}$ C. Schnaible, ${ }^{150}$ B. Stone, ${ }^{150}$ V. Valuev, ${ }^{150}$ K. Burt, ${ }^{151}$ Y. Chen, ${ }^{151}$ R. Clare, ${ }^{151}$ J. W. Gary, ${ }^{151}$ S. M. A. Ghiasi Shirazi, ${ }^{151}$ G. Hanson, ${ }^{151}$ G. Karapostoli, ${ }^{151}$ E. Kennedy, ${ }^{151}$ O. R. Long, ${ }^{151}$ M. Olmedo Negrete, ${ }^{151}$ M. I. Paneva, ${ }^{151}$ W. Si, ${ }^{151}$ L. Wang, ${ }^{151}$ S. Wimpenny, ${ }^{151}$ B. R. Yates, ${ }^{151}$ Y. Zhang, ${ }^{151}$ J. G. Branson, ${ }^{152}$ P. Chang, ${ }^{152}$ S. Cittolin, ${ }^{152}$ S. Cooperstein, ${ }^{152}$ N. Deelen, ${ }^{152}$ M. Derdzinski, ${ }^{152}$ R. Gerosa, ${ }^{152}$ D. Gilbert, ${ }^{152}$ B. Hashemi, ${ }^{152}$ D. Klein, ${ }^{152}$ V. Krutelyov, ${ }^{152}$ J. Letts, ${ }^{152}$ M. Masciovecchio, ${ }^{152}$ S. May, ${ }^{152}$ S. Padhi, ${ }^{152}$ M. Pieri, ${ }^{152}$ V. Sharma, ${ }^{152}$ M. Tadel, ${ }^{152}$ F. Würthwein, ${ }^{152}$ A. Yagil, ${ }^{152}$ G. Zevi Della Porta, ${ }^{152}$ N. Amin, ${ }^{153}$ R. Bhandari, ${ }^{153}$ C. Campagnari, ${ }^{153}$ M. Citron, ${ }^{153}$ V. Dutta, ${ }^{153}$ M. Franco Sevilla, ${ }^{153}$ J. Incandela, ${ }^{153}$ B. Marsh, ${ }^{153}$ H. Mei, ${ }^{153}$ A. Ovcharova, ${ }^{153}$ H. Qu, ${ }^{153}$ J. Richman, ${ }^{153}$ U. Sarica, ${ }^{153}$ D. Stuart, ${ }^{153}$ S. Wang, ${ }^{153}$ D. Anderson, ${ }^{154}$ A. Bornheim, ${ }^{154}$ O. Cerri, ${ }^{154}$ I. Dutta, ${ }^{154}$ J. M. Lawhorn, ${ }^{154}$ N. Lu, ${ }^{154}$ J. Mao, ${ }^{154}$ H. B. Newman, ${ }^{154}$ T. Q. Nguyen, ${ }^{154}$ J. Pata, ${ }^{154}$ M. Spiropulu, ${ }^{154}$ J. R. Vlimant, ${ }^{154}$ S. Xie, ${ }^{154}$ Z. Zhang, ${ }^{154}$ R. Y. Zhu, ${ }^{154}$ M. B. Andrews, ${ }^{155}$ T. Ferguson, ${ }^{155}$ T. Mudholkar, ${ }^{155}$ M. Paulini, ${ }^{155}$ M. Sun, ${ }^{155}$ I. Vorobiev, ${ }^{155}$ M. Weinberg, ${ }^{155}$ J. P. Cumalat, ${ }^{156}$ W. T. Ford, ${ }^{156}$ E. MacDonald, ${ }^{156}$ T. Mulholland, ${ }^{156}$ R. Patel, ${ }^{156}$ A. Perloff, ${ }^{156}$ K. Stenson, ${ }^{156}$ K. A. Ulmer, ${ }^{156}$ S. R. Wagner, ${ }^{156}$ J. Alexander, ${ }^{157}$ Y. Cheng, ${ }^{157}$ J. Chu, ${ }^{157}$ A. Datta, ${ }^{157}$ A. Frankenthal, ${ }^{157}$ K. Mcdermott, ${ }^{157}$ J. R. Patterson, ${ }^{157}$ D. Quach, ${ }^{157}$ M. Reid, ${ }^{157}$ A. Ryd, ${ }^{157}$ S. M. Tan, ${ }^{157}$ Z. Tao, ${ }^{157}$ J. Thom, ${ }^{157}$ P. Wittich, ${ }^{157}$ M. Zientek, ${ }^{157}$ S. Abdullin, ${ }^{158}$ M. Albrow, ${ }^{158}$ M. Alyari, ${ }^{158}$ G. Apollinari, ${ }^{158}$ A. Apresyan, ${ }^{158}$ A. Apyan, ${ }^{158}$ S. Banerjee, ${ }^{158}$ L. A. T. Bauerdick, ${ }^{158}$ A. Beretvas, ${ }^{158}$ D. Berry, ${ }^{158}$ J. Berryhill, ${ }^{158}$ P. C. Bhat, ${ }^{158}$ K. Burkett, ${ }^{158}$ J. N. Butler, ${ }^{158}$ A. Canepa, ${ }^{158}$ G. B. Cerati, ${ }^{158}$ H. W. K. Cheung, ${ }^{158}$ F. Chlebana, ${ }^{158}$ M. Cremonesi, ${ }^{158}$ J. Duarte, ${ }^{158}$ V. D. Elvira, ${ }^{158}$ J. Freeman, ${ }^{158}$ Z. Gecse, ${ }^{158}$ E. Gottschalk, ${ }^{158}$ L. Gray, ${ }^{158}$ D. Green, ${ }^{158}$ S. Grünendahl, ${ }^{158}$ O. Gutsche, ${ }^{158}$ Allison Reinsvold Hall, ${ }^{158} \mathrm{~J}$ Hanlon, ${ }^{158}$ R. M. Harris, ${ }^{158}$ S. Hasegawa, ${ }^{158}$ R. Heller, ${ }^{158}$ J. Hirschauer, ${ }^{158}$ B. Jayatilaka, ${ }^{158}$ S. Jindariani, ${ }^{158}$ M. Johnson, ${ }^{158}$ U. Joshi, ${ }^{158}$ B. Klima, ${ }^{158}$ M. J. Kortelainen, ${ }^{158}$ B. Kreis, ${ }^{158}$ S. Lammel, ${ }^{158}$ J. Lewis, ${ }^{158}$ D. Lincoln, ${ }^{158}$ R. Lipton, ${ }^{158}$ M. Liu, ${ }^{158}$ T. Liu, ${ }^{158}$ J. Lykken, ${ }^{158}$ K. Maeshima, ${ }^{158}$ J. M. Marraffino, ${ }^{158}$ D. Mason, ${ }^{158}$ P. McBride, ${ }^{158}$ P. Merkel, ${ }^{158}$ S. Mrenna, ${ }^{158}$ S. Nahn, ${ }^{158}$ V. O’Dell, ${ }^{158}$ V. Papadimitriou, ${ }^{158}$ K. Pedro, ${ }^{158}$ C. Pena, ${ }^{158}$ G. Rakness, ${ }^{158}$ F. Ravera, ${ }^{158}$ L. Ristori, ${ }^{158}$ B. Schneider, ${ }^{158}$ E. Sexton-Kennedy, ${ }^{158}$ N. Smith, ${ }^{158}$ A. Soha, ${ }^{158}$ W. J. Spalding, ${ }^{158}$ L. Spiegel, ${ }^{158}$ S. Stoynev, ${ }^{158}$ J. Strait, ${ }^{158}$ N. Strobbe, ${ }^{158}$ L. Taylor, ${ }^{158}$ S. Tkaczyk, ${ }^{158}$ N. V. Tran, ${ }^{158}$ L. Uplegger, ${ }^{158}$ E. W. Vaandering, ${ }^{158}$ C. Vernieri, ${ }^{158}$ R. Vidal, ${ }^{158}$ M. Wang, ${ }^{158}$ H. A. Weber, ${ }^{158}$ D. Acosta, ${ }^{159}$ P. Avery, ${ }^{159}$ D. Bourilkov, ${ }^{159}$ A. Brinkerhoff, ${ }^{159}$ L. Cadamuro, ${ }^{159}$ A. Carnes, ${ }^{159}$ V. Cherepanov, ${ }^{159}$ F. Errico, ${ }^{159}$ R. D. Field, ${ }^{159}$ S. V. Gleyzer, ${ }^{159}$ B. M. Joshi, ${ }^{159}$ M. Kim, ${ }^{159}$ J. Konigsberg, ${ }^{159}$ A. Korytov, ${ }^{159}$ K. H. Lo, ${ }^{159}$ P. Ma, ${ }^{159}$ K. Matchev, ${ }^{159}$ N. Menendez, ${ }^{159}$ G. Mitselmakher, ${ }^{159}$ D. Rosenzweig, ${ }^{159}$ K. Shi, ${ }^{159}$ J. Wang, ${ }^{159}$ S. Wang, ${ }^{159}$ X. Zuo, ${ }^{159}$ Y. R. Joshi, ${ }^{160}$ T. Adams, ${ }^{161}$ A. Askew, ${ }^{161}$ S. Hagopian, ${ }^{161}$ V. Hagopian, ${ }^{161}$ K. F. Johnson, ${ }^{161}$ R. Khurana, ${ }^{161}$ T. Kolberg, ${ }^{161}$ G. Martinez, ${ }^{161}$ T. Perry, ${ }^{161}$ H. Prosper, ${ }^{161}$ C. Schiber, ${ }^{161}$ R. Yohay, ${ }^{161}$ J. Zhang, ${ }^{161}$ M. M. Baarmand, ${ }^{162}$ M. Hohlmann, ${ }^{162}$ D. Noonan, ${ }^{162}$ M. Rahmani, ${ }^{162}$ M. Saunders, ${ }^{162}$ F. Yumiceva, ${ }^{162}$ M. R. Adams, ${ }^{163}$ L. Apanasevich, ${ }^{163}$ R. R. Betts, ${ }^{163}$ R. Cavanaugh, ${ }^{163}$ X. Chen, ${ }^{163}$ S. Dittmer, ${ }^{163}$ O. Evdokimov, ${ }^{163}$ C. E. Gerber, ${ }^{163}$ D. A. Hangal, ${ }^{163}$ D. J. Hofman, ${ }^{163}$ K. Jung, ${ }^{163}$ C. Mills, ${ }^{163}$ T. Roy, ${ }^{163}$ M. B. Tonjes, ${ }^{163}$ N. Varelas, ${ }^{163}$ J. Viinikainen, ${ }^{163}$

H. Wang, ${ }^{163}$ X. Wang, ${ }^{163}$ Z. Wu, ${ }^{163}$ M. Alhusseini, ${ }^{164}$ B. Bilki, ${ }^{164, d d d}$ W. Clarida, ${ }^{164}$ K. Dilsiz, ${ }^{164, u u u}$ S. Durgut, ${ }^{164}$ R. P. Gandrajula, ${ }^{164}$ M. Haytmyradov, ${ }^{164}$ V. Khristenko, ${ }^{164}$ O. K. Köseyan, ${ }^{164}$ J.-P. Merlo, ${ }^{164}$ A. Mestvirishvili, ${ }^{164, v v v}$ A. Moeller, ${ }^{164}$ J. Nachtman, ${ }^{164}$ H. Ogul, ${ }^{164, w w w}$ Y. Onel, ${ }^{164}$ F. Ozok, ${ }^{164, x x x}$ A. Penzo, ${ }^{164}$ C. Snyder, ${ }^{164}$ E. Tiras, ${ }^{164}$ J. Wetzel, ${ }^{164}$ B. Blumenfeld, ${ }^{165}$ A. Cocoros, ${ }^{165}$ N. Eminizer, ${ }^{165}$ A. V. Gritsan, ${ }^{165}$ W. T. Hung, ${ }^{165}$ S. Kyriacou, ${ }^{165}$ P. Maksimovic, ${ }^{165}$ J. Roskes, ${ }^{165}$ M. Swartz, ${ }^{165}$ C. Baldenegro Barrera, ${ }^{166}$ P. Baringer, ${ }^{166}$ A. Bean, ${ }^{166}$ S. Boren, ${ }^{166}$ J. Bowen, ${ }^{166}$ A. Bylinkin, ${ }^{166}$ T. Isidori, ${ }^{166}$ S. Khalil, ${ }^{166}$ J. King, ${ }^{166}$ G. Krintiras, ${ }^{166}$ A. Kropivnitskaya, ${ }^{166}$ C. Lindsey, ${ }^{166}$ D. Majumder, ${ }^{166}$ W. Mcbrayer, ${ }^{166}$ N. Minafra, ${ }^{166}$ M. Murray, ${ }^{166}$ C. Rogan, ${ }^{166}$ C. Royon, ${ }^{166}$ S. Sanders, ${ }^{166}$ E. Schmitz, ${ }^{166}$ J. D. Tapia Takaki, ${ }^{166}$ Q. Wang, ${ }^{166}$ J. Williams, ${ }^{166}$ G. Wilson, ${ }^{166}$ S. Duric, ${ }^{167}$ A. Ivanov, ${ }^{167}$ K. Kaadze, ${ }^{167}$ D. Kim, ${ }^{167}$ Y. Maravin, ${ }^{167}$ D. R. Mendis, ${ }^{167}$ T. Mitchell, ${ }^{167}$ A. Modak, ${ }^{167}$ A. Mohammadi, ${ }^{167}$ F. Rebassoo, ${ }^{168}$ D. Wright, ${ }^{168}$ A. Baden, ${ }^{169}$ O. Baron, ${ }^{169}$ A. Belloni, ${ }^{169}$ S. C. Eno, ${ }^{169}$ Y. Feng, ${ }^{169}$ N. J. Hadley, ${ }^{169}$ S. Jabeen, ${ }^{169}$ G. Y. Jeng, ${ }^{169}$ R. G. Kellogg, ${ }^{169}$ J. Kunkle, ${ }^{169}$ A. C. Mignerey, ${ }^{169}$ S. Nabili, ${ }^{169}$ F. Ricci-Tam, ${ }^{169}$ M. Seidel, ${ }^{169}$ Y. H. Shin, ${ }^{169}$ A. Skuja, ${ }^{169}$ S. C. Tonwar, ${ }^{169}$ K. Wong, ${ }^{169}$ D. Abercrombie, ${ }^{170}$ 
B. Allen, ${ }^{170}$ A. Baty, ${ }^{170}$ R. Bi, ${ }^{170}$ S. Brandt, ${ }^{170}$ W. Busza, ${ }^{170}$ I. A. Cali, ${ }^{170}$ M. D’ Alfonso, ${ }^{170}$ G. Gomez Ceballos, ${ }^{170}$ M. Goncharov, ${ }^{170}$ P. Harris, ${ }^{170}$ D. Hsu, ${ }^{170}$ M. Hu, ${ }^{170}$ M. Klute, ${ }^{170}$ D. Kovalskyi, ${ }^{170}$ Y.-J. Lee, ${ }^{170}$ P. D. Luckey, ${ }^{170}$ B. Maier, ${ }^{170}$ A. C. Marini, ${ }^{170}$ C. Mcginn, ${ }^{170}$ C. Mironov, ${ }^{170}$ S. Narayanan, ${ }^{170}$ X. Niu, ${ }^{170}$ C. Paus, ${ }^{170}$ D. Rankin, ${ }^{170}$ C. Roland, ${ }^{170}$ G. Roland, ${ }^{170}$ Z. Shi, ${ }^{170}$ G. S. F. Stephans, ${ }^{170}$ K. Sumorok, ${ }^{170}$ K. Tatar, ${ }^{170}$ D. Velicanu, ${ }^{170}$ J. Wang, ${ }^{170}$ T. W. Wang, ${ }^{170}$ B. Wyslouch, ${ }^{170}$ R. M. Chatterjee, ${ }^{171}$ A. Evans, ${ }^{171}$ S. Guts, ${ }^{171, a}$ P. Hansen, ${ }^{171}$ J. Hiltbrand, ${ }^{171}$ Y. Kubota, ${ }^{171}$ Z. Lesko, ${ }^{171}$ J. Mans, ${ }^{171}$ R. Rusack, ${ }^{171}$ M. A. Wadud, ${ }^{171}$ J. G. Acosta, ${ }^{172}$ S. Oliveros, ${ }^{172}$ K. Bloom, ${ }^{173}$ S. Chauhan, ${ }^{173}$ D. R. Claes, ${ }^{173}$ C. Fangmeier, ${ }^{173}$ L. Finco, ${ }^{173}$ F. Golf, ${ }^{173}$ R. Kamalieddin, ${ }^{173}$ I. Kravchenko, ${ }^{173}$ J. E. Siado, ${ }^{173}$ G. R. Snow, ${ }^{173, a}$ B. Stieger, ${ }^{173}$ W. Tabb, ${ }^{173}$ G. Agarwal, ${ }^{174}$ C. Harrington, ${ }^{174}$ I. Iashvili, ${ }^{174}$ A. Kharchilava, ${ }^{174}$ C. McLean, ${ }^{174}$ D. Nguyen, ${ }^{174}$ A. Parker, ${ }^{174}$ J. Pekkanen, ${ }^{174}$ S. Rappoccio, ${ }^{174}$ B. Roozbahani, ${ }^{174}$ G. Alverson, ${ }^{175}$ E. Barberis, ${ }^{175}$ C. Freer, ${ }^{175}$ Y. Haddad, ${ }^{175}$ A. Hortiangtham, ${ }^{175}$ G. Madigan, ${ }^{175}$ B. Marzocchi, ${ }^{175}$ D. M. Morse, ${ }^{175}$ T. Orimoto, ${ }^{175}$ L. Skinnari, ${ }^{175}$ A. Tishelman-Charny, ${ }^{175}$ T. Wamorkar, ${ }^{175}$ B. Wang, ${ }^{175}$ A. Wisecarver, ${ }^{175}$ D. Wood, ${ }^{175}$ S. Bhattacharya, ${ }^{176}$ J. Bueghly, ${ }^{176}$ T. Gunter, ${ }^{176}$ K. A. Hahn, ${ }^{176}$ N. Odell, ${ }^{176}$ M. H. Schmitt, ${ }^{176}$ K. Sung, ${ }^{176}$ M. Trovato, ${ }^{176}$ M. Velasco, ${ }^{176}$ R. Bucci, ${ }^{177}$ N. Dev, ${ }^{177}$ R. Goldouzian, ${ }^{177}$ M. Hildreth, ${ }^{177}$ K. Hurtado Anampa, ${ }^{177}$ C. Jessop, ${ }^{177}$ D. J. Karmgard, ${ }^{177}$ K. Lannon, ${ }^{177}$ W. Li, ${ }^{177}$ N. Loukas, ${ }^{177}$ N. Marinelli, ${ }^{177}$ I. Mcalister, ${ }^{177}$ F. Meng, ${ }^{177}$ C. Mueller, ${ }^{177}$ Y. Musienko, ${ }^{177,11}$ M. Planer, ${ }^{177}$ R. Ruchti, ${ }^{177}$ P. Siddireddy, ${ }^{177}$ G. Smith, ${ }^{177}$ S. Taroni, ${ }^{177}$ M. Wayne, ${ }^{177}$ A. Wightman, ${ }^{177}$ M. Wolf, ${ }^{177}$ A. Woodard, ${ }^{177}$ J. Alimena, ${ }^{178}$ B. Bylsma, ${ }^{178}$ L. S. Durkin, ${ }^{178}$ B. Francis, ${ }^{178}$ C. Hill, ${ }^{178}$ W. Ji, ${ }^{178}$ A. Lefeld, ${ }^{178}$ T. Y. Ling, ${ }^{178}$ B. L. Winer, ${ }^{178}$ G. Dezoort, ${ }^{179}$ P. Elmer, ${ }^{179}$ J. Hardenbrook, ${ }^{179}$ N. Haubrich, ${ }^{179}$ S. Higginbotham, ${ }^{179}$ A. Kalogeropoulos, ${ }^{179}$ S. Kwan, ${ }^{179}$ D. Lange, ${ }^{179}$ M. T. Lucchini, ${ }^{179}$ J. Luo, ${ }^{179}$ D. Marlow, ${ }^{179}$ K. Mei, ${ }^{179}$ I. Ojalvo, ${ }^{179}$ J. Olsen, ${ }^{179}$ C. Palmer, ${ }^{179}$ P. Piroué, ${ }^{179}$

J. Salfeld-Nebgen, ${ }^{179}$ D. Stickland, ${ }^{179}$ C. Tully, ${ }^{179}$ Z. Wang, ${ }^{179}$ S. Malik, ${ }^{180}$ S. Norberg, ${ }^{180}$ A. Barker, ${ }^{181}$ V. E. Barnes, ${ }^{181}$ S. Das, ${ }^{181}$ L. Gutay, ${ }^{181}$ M. Jones, ${ }^{181}$ A. W. Jung, ${ }^{181}$ A. Khatiwada, ${ }^{181}$ B. Mahakud, ${ }^{181}$ D. H. Miller, ${ }^{181}$ G. Negro, ${ }^{181}$ N. Neumeister, ${ }^{181}$ C. C. Peng, ${ }^{181}$ S. Piperov, ${ }^{181}$ H. Qiu, ${ }^{181}$ J. F. Schulte, ${ }^{181}$ N. Trevisani, ${ }^{181}$ F. Wang, ${ }^{181}$ R. Xiao, ${ }^{181}$ W. Xie, ${ }^{181}$ T. Cheng, ${ }^{182}$ J. Dolen, ${ }^{182}$ N. Parashar, ${ }^{182}$ U. Behrens, ${ }^{183}$ K. M. Ecklund, ${ }^{183}$ S. Freed, ${ }^{183}$ F. J. M. Geurts, ${ }^{183}$ M. Kilpatrick, ${ }^{183}$ Arun Kumar, ${ }^{183}$ W. Li, ${ }^{183}$ B. P. Padley, ${ }^{183}$ R. Redjimi, ${ }^{183}$ J. Roberts, ${ }^{183}$ J. Rorie, ${ }^{183}$ W. Shi, ${ }^{183}$ A. G. Stahl Leiton, ${ }^{183}$ Z. Tu, ${ }^{183}$ A. Zhang, ${ }^{183}$ A. Bodek, ${ }^{184}$ P. de Barbaro, ${ }^{184}$ R. Demina, ${ }^{184}$ J. L. Dulemba, ${ }^{184}$ C. Fallon, ${ }^{184}$ T. Ferbel, ${ }^{184}$ M. Galanti, ${ }^{184}$ A. Garcia-Bellido, ${ }^{184}$ O. Hindrichs, ${ }^{184}$ A. Khukhunaishvili, ${ }^{184}$ E. Ranken, ${ }^{184}$ R. Taus, ${ }^{184}$ B. Chiarito, ${ }^{185}$ J. P. Chou, ${ }^{185}$ A. Gandrakota, ${ }^{185}$ Y. Gershtein, ${ }^{185}$ E. Halkiadakis, ${ }^{185}$ A. Hart, ${ }^{185}$ M. Heindl, ${ }^{185}$ E. Hughes, ${ }^{185}$ S. Kaplan, ${ }^{185}$ I. Laflotte, ${ }^{185}$ A. Lath, ${ }^{185}$ R. Montalvo, ${ }^{185}$ K. Nash, ${ }^{185}$ M. Osherson, ${ }^{185}$ H. Saka, ${ }^{185}$ S. Salur, ${ }^{185}$ S. Schnetzer, ${ }^{185}$ S. Somalwar, ${ }^{185}$

R. Stone, ${ }^{185}$ S. Thomas, ${ }^{185}$ H. Acharya, ${ }^{186}$ A. G. Delannoy, ${ }^{186}$ S. Spanier, ${ }^{186}$ O. Bouhali, ${ }^{187, y y y}$ M. Dalchenko, ${ }^{187}$ M. De Mattia, ${ }^{187}$ A. Delgado, ${ }^{187}$ S. Dildick, ${ }^{187}$ R. Eusebi, ${ }^{187}$ J. Gilmore, ${ }^{187}$ T. Huang, ${ }^{187}$ T. Kamon, ${ }^{187, z z z}$ S. Luo, ${ }^{187}$ S. Malhotra, ${ }^{187}$ D. Marley, ${ }^{187}$ R. Mueller, ${ }^{187}$ D. Overton, ${ }^{187}$ L. Perniè, ${ }^{187}$ D. Rathjens, ${ }^{187}$ A. Safonov, ${ }^{187}$ N. Akchurin, ${ }^{188}$ J. Damgov, ${ }^{188}$ F. De Guio, ${ }^{188}$ S. Kunori, ${ }^{188}$ K. Lamichhane, ${ }^{188}$ S. W. Lee, ${ }^{188}$ T. Mengke, ${ }^{188}$ S. Muthumuni, ${ }^{188}$ T. Peltola, ${ }^{188}$ S. Undleeb, ${ }^{188}$ I. Volobouev, ${ }^{188}$ Z. Wang, ${ }^{188}$ A. Whitbeck, ${ }^{188}$ S. Greene, ${ }^{189}$ A. Gurrola, ${ }^{189}$ R. Janjam, ${ }^{189}$ W. Johns, ${ }^{189}$ C. Maguire, ${ }^{189}$ A. Melo, ${ }^{189}$ H. Ni, ${ }^{189}$ K. Padeken, ${ }^{189}$ F. Romeo, ${ }^{189}$ P. Sheldon, ${ }^{189}$ S. Tuo, ${ }^{189}$ J. Velkovska, ${ }^{189}$ M. Verweij, ${ }^{189}$ M. W. Arenton, ${ }^{190}$ P. Barria, ${ }^{190}$ B. Cox, ${ }^{190}$ G. Cummings, ${ }^{190}$ J. Hakala, ${ }^{190}$ R. Hirosky, ${ }^{190}$ M. Joyce, ${ }^{190}$ A. Ledovskoy, ${ }^{190}$ C. Neu, ${ }^{190}$ B. Tannenwald, ${ }^{190}$ Y. Wang, ${ }^{190}$ E. Wolfe, ${ }^{190}$ F. Xia, ${ }^{190}$ R. Harr, ${ }^{191}$ P. E. Karchin, ${ }^{191}$ N. Poudyal, ${ }^{191}$ J. Sturdy, ${ }^{191}$ P. Thapa, ${ }^{191}$ T. Bose, ${ }^{192}$ J. Buchanan, ${ }^{192}$ C. Caillol, ${ }^{192}$ D. Carlsmith, ${ }^{192}$ S. Dasu, ${ }^{192}$ I. De Bruyn, ${ }^{192}$ L. Dodd, ${ }^{192}$ F. Fiori, ${ }^{192}$ C. Galloni, ${ }^{192}$ B. Gomber, ${ }^{192, a a a a}$ H. He, ${ }^{192}$ M. Herndon, ${ }^{192}$ A. Hervé, ${ }^{192}$ U. Hussain, ${ }^{192}$ P. Klabbers, ${ }^{192}$ A. Lanaro, ${ }^{192}$ A. Loeliger, ${ }^{192}$ K. Long, ${ }^{192}$ R. Loveless, ${ }^{192}$ J. Madhusudanan Sreekala, ${ }^{192}$ D. Pinna, ${ }^{192}$ T. Ruggles, ${ }^{192}$ A. Savin, ${ }^{192}$ V. Sharma, ${ }^{192}$ W. H. Smith, ${ }^{192}$ D. Teague, ${ }^{192}$ S. Trembath-reichert, ${ }^{192}$ and N. Woods ${ }^{192}$

(CMS Collaboration)

${ }^{1}$ Yerevan Physics Institute, Yerevan, Armenia

${ }^{2}$ Institut für Hochenergiephysik, Wien, Austria

${ }^{3}$ Institute for Nuclear Problems, Minsk, Belarus

${ }^{4}$ Universiteit Antwerpen, Antwerpen, Belgium

${ }^{5}$ Vrije Universiteit Brussel, Brussel, Belgium

${ }^{6}$ Université Libre de Bruxelles, Bruxelles, Belgium

${ }^{7}$ Ghent University, Ghent, Belgium

${ }^{8}$ Université Catholique de Louvain, Louvain-la-Neuve, Belgium

${ }^{9}$ Centro Brasileiro de Pesquisas Fisicas, Rio de Janeiro, Brazil 


\footnotetext{
${ }^{10}$ Universidade do Estado do Rio de Janeiro, Rio de Janeiro, Brazil

${ }^{11}$ Universidade Estadual Paulista, Universidade Federal do ABC, São Paulo, Brazil

${ }^{11 a}$ Universidade Estadual Paulista, Santo André, São Paulo, Brazil

${ }^{11 \mathrm{~b}}$ Universidade Federal do ABC, São Paulo, Brazil

${ }^{12}$ Institute for Nuclear Research and Nuclear Energy, Bulgarian Academy of Sciences, Sofia, Bulgaria

${ }^{13}$ University of Sofia, Sofia, Bulgaria

${ }^{14}$ Beihang University, Beijing, China

${ }^{15}$ Institute of High Energy Physics, Beijing, China

${ }^{16}$ State Key Laboratory of Nuclear Physics and Technology, Peking University, Beijing, China

${ }^{17}$ Tsinghua University, Beijing, China

${ }^{18}$ Zhejiang University, Hangzhou, China

${ }^{19}$ Universidad de Los Andes, Bogota, Colombia

${ }^{20}$ Universidad de Antioquia, Medellin, Colombia

${ }^{21}$ University of Split, Faculty of Electrical Engineering, Mechanical Engineering and Naval Architecture, Split, Croatia

${ }^{22}$ University of Split, Faculty of Science, Split, Croatia

${ }^{23}$ Institute Rudjer Boskovic, Zagreb, Croatia

${ }^{24}$ University of Cyprus, Nicosia, Cyprus

${ }^{25}$ Charles University, Prague, Czech Republic

${ }^{26}$ Escuela Politecnica Nacional, Quito, Ecuador

${ }^{27}$ Universidad San Francisco de Quito, Quito, Ecuador

${ }^{28}$ Academy of Scientific Research and Technology of the Arab Republic of Egypt, Egyptian Network of High Energy Physics, Cairo, Egypt

${ }^{29}$ National Institute of Chemical Physics and Biophysics, Tallinn, Estonia

${ }^{30}$ Department of Physics, University of Helsinki, Helsinki, Finland

${ }^{31}$ Helsinki Institute of Physics, Helsinki, Finland

${ }^{32}$ Lappeenranta University of Technology, Lappeenranta, Finland

${ }^{33}$ IRFU, CEA, Université Paris-Saclay, Gif-sur-Yvette, France

${ }^{34}$ Laboratoire Leprince-Ringuet, Ecole polytechnique, CNRS/IN2P3, Université Paris-Saclay, Palaiseau, France

${ }^{35}$ Université de Strasbourg, CNRS, IPHC UMR 7178, Strasbourg, France

${ }^{36}$ Centre de Calcul de l'Institut National de Physique Nucleaire et de Physique des Particules, CNRS/IN2P3, Villeurbanne, France

${ }^{37}$ Université de Lyon, Université Claude Bernard Lyon 1, CNRS-IN2P3, Institut de Physique Nucléaire de Lyon, Villeurbanne, France

${ }^{38}$ Georgian Technical University, Tbilisi, Georgia

${ }^{39}$ Tbilisi State University, Tbilisi, Georgia

${ }^{40}$ RWTH Aachen University, I. Physikalisches Institut, Aachen, Germany

${ }^{41}$ RWTH Aachen University, III. Physikalisches Institut A, Aachen, Germany

${ }^{42}$ RWTH Aachen University, III. Physikalisches Institut B, Aachen, Germany

${ }^{43}$ Deutsches Elektronen-Synchrotron, Hamburg, Germany

${ }^{44}$ University of Hamburg, Hamburg, Germany

${ }^{45}$ Karlsruher Institut fuer Technologie, Karlsruhe, Germany

${ }^{46}$ Institute of Nuclear and Particle Physics (INPP), NCSR Demokritos, Aghia Paraskevi, Greece

${ }^{47}$ National and Kapodistrian University of Athens, Athens, Greece

${ }^{48}$ National Technical University of Athens, Athens, Greece

${ }^{49}$ University of Ioánnina, Ioánnina, Greece

${ }^{50}$ MTA-ELTE Lendület CMS Particle and Nuclear Physics Group, Eötvös Loránd University, Budapest, Hungary

${ }^{51}$ Wigner Research Centre for Physics, Budapest, Hungary

${ }^{52}$ Institute of Nuclear Research ATOMKI, Debrecen, Hungary

${ }^{53}$ Institute of Physics, University of Debrecen, Debrecen, Hungary

${ }^{54}$ Eszterhazy Karoly University, Karoly Robert Campus, Gyongyos, Hungary

${ }^{55}$ Indian Institute of Science (IISc), Bangalore, India

${ }^{56}$ National Institute of Science Education and Research, HBNI, Bhubaneswar, India

${ }^{57}$ Panjab University, Chandigarh, India

${ }^{58}$ University of Delhi, Delhi, India

${ }^{59}$ Saha Institute of Nuclear Physics, HBNI, Kolkata,India

${ }^{60}$ Indian Institute of Technology Madras, Madras, India

${ }^{61}$ Bhabha Atomic Research Centre, Mumbai, India
} 
${ }^{62}$ Tata Institute of Fundamental Research-A, Mumbai, India

${ }^{63}$ Tata Institute of Fundamental Research-B, Mumbai, India

${ }^{64}$ Indian Institute of Science Education and Research (IISER), Pune, India

${ }^{65}$ Institute for Research in Fundamental Sciences (IPM), Tehran, Iran

${ }^{66}$ University College Dublin, Dublin, Ireland

${ }^{67 a}$ INFN Sezione di Bari, Bari, Italy

${ }^{67 \mathrm{~b}}$ Università di Bari, Bari, Italy

${ }^{67 \mathrm{c}}$ Politecnico di Bari, Bari, Italy

${ }^{68}$ INFN Sezione di Bologna, Università di Bologna, Bologna, Italy

${ }^{68 \mathrm{a}}$ INFN Sezione di Bologna, Bologna, Italy

${ }^{68 \mathrm{~b}}$ Università di Bologna, Bologna, Italy

${ }^{69}$ INFN Sezione di Catania, Università di Catania, Catania, Italy

${ }^{69 a}$ INFN Sezione di Catania, Catania, Italy

${ }^{69 \mathrm{~b}}$ Università di Catania, Catania, Italy

${ }^{70}$ INFN Sezione di Firenze, Università di Firenze, Firenze, Italy

${ }^{70 a}$ INFN Sezione di Firenze, Firenze, Italy

${ }^{70 \mathrm{~b}}$ Università di Firenze, Firenze, Italy

${ }^{71}$ INFN Laboratori Nazionali di Frascati, Frascati, Italy

${ }^{72}$ INFN Sezione di Genova, Università di Genova, Genova, Italy

${ }^{72 a}$ INFN Sezione di Genova, Genova, Italy

${ }^{72 \mathrm{~b}}$ Università di Genova, Genova, Italy

${ }^{73}$ INFN Sezione di Milano-Bicocca, Università di Milano-Bicocca, Milano, Italy

${ }^{73 a}$ INFN Sezione di Milano-Bicocca, Milano, Italy

${ }^{73 \mathrm{~b}}$ Università di Milano-Bicocca, Milano, Italy

${ }^{74}$ INFN Sezione di Napoli, Università di Napoli 'Federico II', Napoli, Italy, Università della Basilicata,

Potenza, Italy, and Università G. Marconi, Roma, Italy

${ }^{74 a}$ INFN Sezione di Napoli, Naples, Italy

${ }^{74 b}$ Università di Napoli 'Federico II', Naples, Italy

${ }^{74 c}$ Università della Basilicata, Potenza, Italy

${ }^{74 d}$ Università G. Marconi, Roma, Italy

${ }^{75}$ INFN Sezione di Padova, Università di Padova, Padova, Italy and Università di Trento, Trento, Italy

${ }^{75 a}$ INFN Sezione di Padova, Padova, Italy

${ }^{75 b}$ Università di Padova, Padova, Italy

${ }^{75 \mathrm{c}}$ Università di Trento, Tento, Italy

${ }^{76 a}$ INFN Sezione di Pavia, Pavia, Italy

${ }^{76 \mathrm{~b}}$ Università di Pavia, Pavia, Italy

${ }^{77}$ INFN Sezione di Perugia, Università di Perugia, Perugia, Italy

${ }^{77 a}$ INFN Sezione di Perugia

${ }^{77 b}$ Università di Perugia

${ }^{78}$ INFN Sezione di Pisa, Università di Pisa, Scuola Normale Superiore di Pisa, Pisa, Italy

${ }^{78 a}$ INFN Sezione di Pisa, Pisa, Italy

${ }^{78 b}$ Università di Pisa, Pisa, Italy

${ }^{78 c}$ Scuola Normale Superiore di Pisa, Pisa, Italy

${ }^{79}$ INFN Sezione di Roma, Sapienza Università di Roma, Rome, Italy

${ }^{79 a}$ INFN Sezione di Roma, Roma, Italy

${ }^{79 b}$ Sapienza Università di Roma, Roma, Italy

${ }^{80}$ INFN Sezione di Torino, Università di Torino, Torino, Italy, Università del Piemonte Orientale, Novara, Italy

${ }^{80 \mathrm{a}}$ INFN Sezione di Torino, Torino, Italy

${ }^{80 \mathrm{~b}}$ Università di Torino, Torino, Italy

${ }^{80 \mathrm{c}}$ Università del Piemonte Orientale, Novara, Italy

${ }^{81}$ INFN Sezione di Trieste, Università di Trieste, Trieste, Italy

${ }^{81 a}$ INFN Sezione di Trieste, Trieste, Italy

${ }^{81 \mathrm{~b}}$ Università di Trieste, Trieste, Italy

${ }^{82}$ Kyungpook National University, Daegu, Korea

${ }^{83}$ Chonnam National University, Institute for Universe and Elementary Particles, Kwangju, Korea

${ }^{84}$ Hanyang University, Seoul, Korea

${ }^{85}$ Korea University, Seoul, Korea

${ }^{86}$ Kyung Hee University, Department of Physics, Seoul, Korea

${ }^{87}$ Sejong University, Seoul, Korea 
${ }^{88}$ Seoul National University, Seoul, Korea

${ }^{89}$ University of Seoul, Seoul, Korea

${ }^{90}$ Sungkyunkwan University, Suwon, Korea

${ }^{91}$ Riga Technical University, Riga, Latvia

${ }^{92}$ Vilnius University, Vilnius, Lithuania

${ }^{93}$ National Centre for Particle Physics, Universiti Malaya, Kuala Lumpur, Malaysia

${ }^{94}$ Universidad de Sonora (UNISON), Hermosillo, Mexico

${ }^{95}$ Centro de Investigacion y de Estudios Avanzados del IPN, Mexico City, Mexico

${ }^{96}$ Universidad Iberoamericana, Mexico City, Mexico

${ }^{97}$ Benemerita Universidad Autonoma de Puebla, Puebla, Mexico

${ }^{98}$ Universidad Autónoma de San Luis Potosí, San Luis Potosí, Mexico

${ }^{99}$ University of Montenegro, Podgorica, Montenegro

${ }^{100}$ University of Auckland, Auckland, New Zealand

${ }^{101}$ University of Canterbury, Christchurch, New Zealand

${ }^{102}$ National Centre for Physics, Quaid-I-Azam University, Islamabad, Pakistan

${ }^{103}$ AGH University of Science and Technology Faculty of Computer Science,

Electronics and Telecommunications, Krakow, Poland

${ }^{104}$ National Centre for Nuclear Research, Swierk, Poland

${ }^{105}$ Institute of Experimental Physics, Faculty of Physics, University of Warsaw, Warsaw, Poland

${ }^{106}$ Laboratório de Instrumentação e Física Experimental de Partículas, Lisboa, Portugal

${ }^{107}$ Joint Institute for Nuclear Research, Dubna, Russia

${ }^{108}$ Petersburg Nuclear Physics Institute, Gatchina (St. Petersburg), Russia

${ }^{109}$ Institute for Nuclear Research, Moscow, Russia

${ }^{110}$ Institute for Theoretical and Experimental Physics named by A.I. Alikhanov of NRC

'Kurchatov Institute', Moscow, Russia

${ }^{111}$ Moscow Institute of Physics and Technology,

Moscow, Russia

${ }^{112}$ National Research Nuclear University 'Moscow Engineering Physics Institute' (MEPhI), Moscow, Russia

${ }^{113}$ P.N. Lebedev Physical Institute, Moscow, Russia

${ }^{114}$ Skobeltsyn Institute of Nuclear Physics, Lomonosov Moscow State University, Moscow, Russia

${ }^{115}$ Novosibirsk State University (NSU), Novosibirsk, Russia

${ }^{116}$ Institute for High Energy Physics of National Research Centre 'Kurchatov Institute', Protvino, Russia

${ }^{117}$ National Research Tomsk Polytechnic University, Tomsk, Russia

${ }^{118}$ Tomsk State University, Tomsk, Russia

${ }^{119}$ University of Belgrade, Faculty of Physics and VINCA Institute of Nuclear Sciences, Belgrade, Serbia

${ }^{120}$ Centro de Investigaciones Energéticas Medioambientales y Tecnológicas (CIEMAT), Madrid, Spain

${ }^{121}$ Universidad Autónoma de Madrid, Madrid, Spain

${ }^{122}$ Universidad de Oviedo, Instituto Universitario de Ciencias y Tecnologías Espaciales de Asturias (ICTEA), Oviedo, Spain

${ }^{123}$ Instituto de Física de Cantabria (IFCA), CSIC-Universidad de Cantabria, Santander, Spain

${ }^{124}$ University of Colombo, Colombo, Sri Lanka

${ }^{125}$ University of Ruhuna, Department of Physics, Matara, Sri Lanka

${ }^{126}$ CERN, European Organization for Nuclear Research, Geneva, Switzerland

${ }^{127}$ Paul Scherrer Institut, Villigen, Switzerland

${ }^{128}$ ETH Zurich-Institute for Particle Physics and Astrophysics (IPA), Zurich, Switzerland

${ }^{129}$ Universität Zürich, Zurich, Switzerland

${ }^{130}$ National Central University, Chung-Li, Taiwan

${ }^{131}$ National Taiwan University (NTU), Taipei, Taiwan

${ }^{132}$ Chulalongkorn University, Faculty of Science, Department of Physics, Bangkok, Thailand

${ }^{133}$ Cukurova University, Physics Department, Science and Art Faculty, Adana, Turkey

${ }^{134}$ Middle East Technical University, Physics Department, Ankara, Turkey

${ }^{135}$ Bogazici University, Istanbul, Turkey

${ }^{136}$ Istanbul Technical University, Istanbul, Turkey

${ }^{137}$ Istanbul University, Istanbul, Turkey

${ }^{138}$ Institute for Scintillation Materials of National Academy of Science of Ukraine, Kharkov, Ukraine

${ }^{139}$ National Scientific Center, Kharkov Institute of Physics and Technology, Kharkov, Ukraine

${ }^{140}$ University of Bristol, Bristol, United Kingdom

${ }^{141}$ Rutherford Appleton Laboratory, Didcot, United Kingdom

${ }^{142}$ Imperial College, London, United Kingdom 


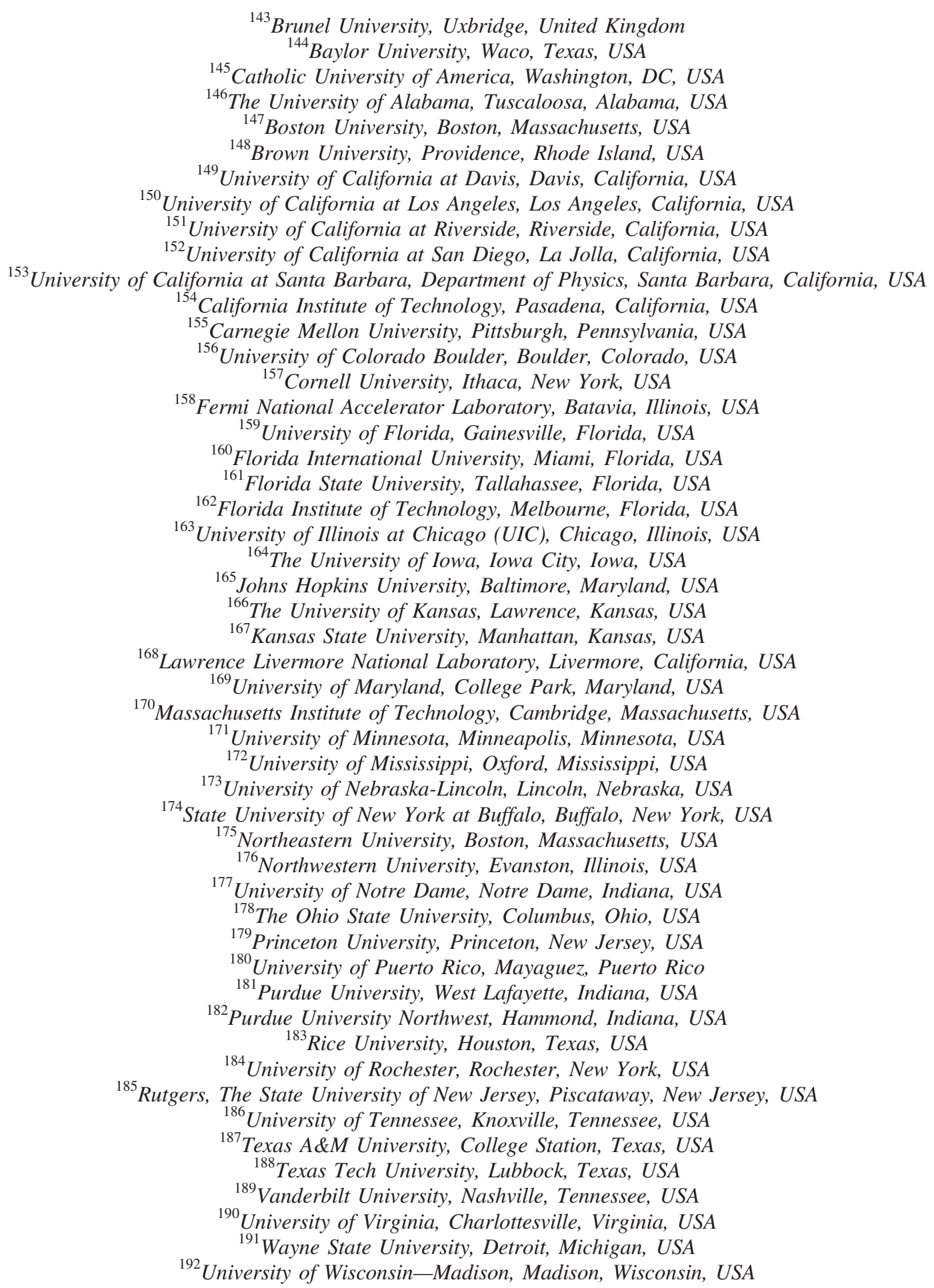

${ }^{\mathrm{a}}$ Deceased.

${ }^{\mathrm{b}}$ Also at Vienna University of Technology, Vienna, Austria.

${ }^{\mathrm{c}}$ Also at IRFU, CEA, Université Paris-Saclay, Gif-sur-Yvette, France.

${ }^{\mathrm{d}}$ Also at Universidade Estadual de Campinas, Campinas, Brazil.

${ }^{\mathrm{e}}$ Also at Federal University of Rio Grande do Sul, Porto Alegre, Brazil.

${ }_{\mathrm{f}}^{\mathrm{A}}$ Also at Fundação Universidad Federal de Mato Grosso do Sul, Mato Grosso do Sul, Brazil.

${ }^{\mathrm{g}}$ Also at Universidade Federal de Pelotas, Pelotas, Brazil.

${ }^{\mathrm{h}}$ Also at Université Libre de Bruxelles, Bruxelles, Belgium.

${ }^{\mathrm{i}}$ Also at University of Chinese Academy of Sciences, Beijing, China. 
${ }^{\mathrm{j}}$ Also at Institute for Theoretical and Experimental Physics named by A.I. Alikhanov of NRC 'Kurchatov Institute', Moscow, Russia.

${ }^{\mathrm{k}}$ Also at Joint Institute for Nuclear Research, Dubna, Russia.

${ }^{1}$ Also at British University in Egypt, Cairo, Egypt.

${ }^{\mathrm{m}}$ Also at Suez University, Suez, Egypt.

${ }^{\mathrm{n}}$ Also at Purdue University, West Lafayette, Indiana, USA.

${ }^{\circ}$ Also at Université de Haute Alsace, Mulhouse, France.

${ }^{\mathrm{p}}$ Also at Tbilisi State University, Tbilisi, Georgia.

${ }^{\mathrm{q}}$ Also at Erzincan Binali Yildirim University, Erzincan, Turkey.

${ }^{\mathrm{r}}$ Also at CERN, European Organization for Nuclear Research, Geneva, Switzerland.

${ }^{\mathrm{s}}$ Also at RWTH Aachen University, III. Physikalisches Institut A, Aachen, Germany.

${ }^{\mathrm{t}}$ Also at University of Hamburg, Hamburg, Germany.

${ }^{u}$ Also at Brandenburg University of Technology, Cottbus, Germany.

${ }^{v}$ Also at Institute of Physics, University of Debrecen, Debrecen, Hungary.

${ }^{\mathrm{w}}$ Also at Institute of Nuclear Research ATOMKI, Debrecen, Hungary.

${ }^{x}$ Also at MTA-ELTE Lendület CMS Particle and Nuclear Physics Group, Eötvös Loránd University, Budapest, Hungary.

${ }^{\mathrm{y}}$ Also at IIT Bhubaneswar, Bhubaneswar, India.

${ }^{\mathrm{z}}$ Also at Institute of Physics, Bhubaneswar, India.

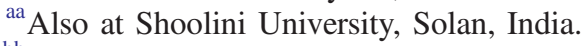

${ }^{\mathrm{bb}}$ Also at University of Visva-Bharati, Santiniketan, India.

${ }^{\mathrm{cc}}$ Also at Isfahan University of Technology, Isfahan, Iran.

${ }^{\text {dd }}$ Also at INFN Sezione di Bari, Università di Bari, Politecnico di Bari, Bari, Italy.

${ }^{e e}$ Also at Italian National Agency for New Technologies, Energy and Sustainable Economic Development, Bologna, Italy.

${ }^{\mathrm{ff}}$ Also at Centro Siciliano di Fisica Nucleare e di Struttura Della Materia, Catania, Italy.

${ }^{g g}$ Also at Scuola Normale e Sezione dell'INFN, Pisa, Italy.

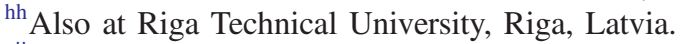

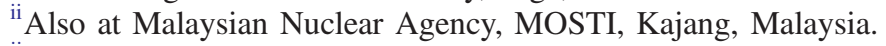

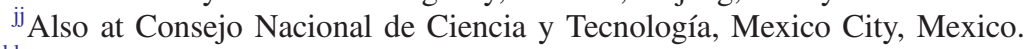

${ }^{\mathrm{kk}}$ Also at Warsaw University of Technology, Institute of Electronic Systems, Warsaw, Poland.

${ }^{11}$ Also at Institute for Nuclear Research, Moscow, Russia.

${ }^{\mathrm{mm}}$ Also at National Research Nuclear University 'Moscow Engineering Physics Institute' (MEPhI), Moscow, Russia.

${ }^{\mathrm{nn}}$ Also at St. Petersburg State Polytechnical University, St. Petersburg, Russia.

${ }^{\text {oo }}$ Also at University of Florida, Gainesville, Florida, USA.

${ }^{\mathrm{pp}}$ Also at Imperial College, London, United Kingdom.

${ }^{\mathrm{qq}}$ Also at P.N. Lebedev Physical Institute, Moscow, Russia.

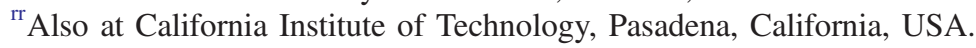

${ }^{\text {ss }}$ Also at Budker Institute of Nuclear Physics, Novosibirsk, Russia.

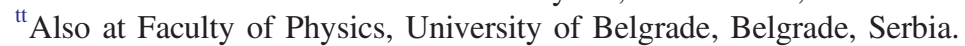

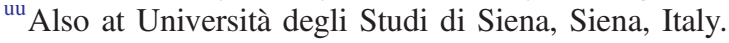

${ }^{v v}$ Also at INFN Sezione di Pavia, Università di Pavia, Pavia, Italy.

${ }^{w w}$ Also at National and Kapodistrian University of Athens, Athens, Greece.

${ }^{\mathrm{xx}}$ Also at Universität Zürich, Zurich, Switzerland.

${ }^{\text {yy }}$ Also at Stefan Meyer Institute for Subatomic Physics, Vienna, Austria.

${ }^{\mathrm{zz}}$ Also at Burdur Mehmet Akif Ersoy University.

${ }^{\text {aaa }}$ Also at Adiyaman University, Adiyaman, Turkey.

${ }^{b b b}$ Also at Şırnak University, Şırnak, Turkey.

${ }^{c c c}$ Also at Tsinghua University, Beijing, China.

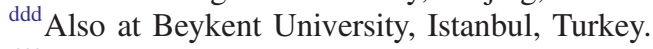

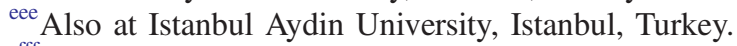

${ }^{\mathrm{fff}}$ Also at Mersin University, Mersin, Turkey.

${ }^{\text {ggg }}$ Also at Piri Reis University, Istanbul, Turkey.

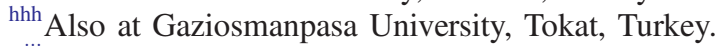

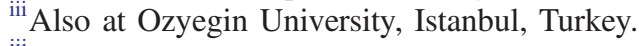

${ }^{\mathrm{jjj}}$ Also at Izmir Institute of Technology, Izmir, Turkey.

${ }^{k k k}$ Also at Marmara University, Istanbul, Turkey.

${ }^{11 l}$ Also at Kafkas University, Kars, Turkey.

${ }^{\mathrm{mmm}}$ Also at Istanbul Bilgi University, Istanbul, Turkey.

${ }^{n n n}$ Also at Hacettepe University, Ankara, Turkey.

${ }^{\circ 00}$ Also at Vrije Universiteit Brussel, Brussel, Belgium

${ }^{\mathrm{ppp}}$ Also at School of Physics and Astronomy, University of Southampton, Southampton, United Kingdom.

${ }^{\mathrm{qqq}}$ Also at IPPP Durham University, Durham, England. 
${ }^{\mathrm{rrr}}$ Also at Monash University, Faculty of Science, Clayton, Australia.

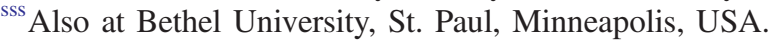

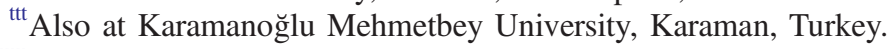

uuu Also at Bingol University, Bingol, Turkey.

${ }^{\mathrm{vvv}}$ Also at Georgian Technical University, Tbilisi, Georgia.

${ }^{\text {www }}$ Also at Sinop University, Sinop, Turkey.

${ }^{x x x}$ Also at Mimar Sinan University, Istanbul, Istanbul, Turkey.

${ }^{\text {yyy }}$ Also at Texas A\&M University at Qatar, Doha, Qatar.

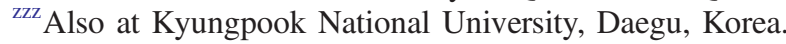

${ }^{\text {aaaa }}$ Also at University of Hyderabad, Hyderabad, India. 